\title{
Neuronal mitochondria transport Pink1 mRNA via Synaptojanin 2 to support local
}

\section{mitophagy}

Angelika B. Harbauer ${ }^{1,2,3,4,5, *}$, Simone Wanderoy ${ }^{3}$, J. Tabitha Hees ${ }^{3}$, Whitney Gibbs ${ }^{1,2}$, Martha Ordonez ${ }^{1,2}$, Zerong Cai ${ }^{1,2}$, Romain Cartoni ${ }^{1,2}$, Ghazaleh Ashrafi ${ }^{1,2}$, Chen Wang ${ }^{1,2}$, Zhigang $\mathrm{He}^{1,2}$, Thomas L. Schwarz ${ }^{1,2,6, *}$

${ }^{1}$ F.M. Kirby Neurobiology Center, Boston Children's Hospital, 300 Longwood Avenue, Boston, MA 02115, USA

${ }^{2}$ Department of Neurobiology, Harvard Medical School, Boston, MA 02115, USA

${ }^{3}$ Max Planck Institute for Neurobiology, Am Klopferspitz 18, 82152 Martinsried, Germany

${ }^{4}$ Technical University of Munich, Institute of Neuronal Cell Biology, Biedersteiner Straße 29, 80802 Munich, Germany

${ }^{5}$ Munich Cluster of Systems Neurology (SyNergy), Feodor-Lynen-Straße 17, 81377 Munich, Germany

${ }^{6}$ Lead Contact

* Correspondence: Angelika.Harbauer@neuro.mpg.de (A.B.H.),

Thomas.schwarz@childrens.harvard.edu (T.L.S.)

\section{Abstract}

PTEN-induced kinase 1 (PINK1) is a very short-lived protein that is required for the removal of damaged mitochondria through Parkin translocation and mitophagy. Because the short halflife of PINK1 limits its ability to be trafficked into neurites, local translation is required for this mitophagy pathway to be active far from the soma. The Pink 1 transcript is associated with and cotransported with neuronal mitochondria. In concert with translation, the mitochondrial outer membrane protein Synaptojanin 2 binding protein (SYNJ2BP) and Synaptojanin 2 (SYNJ2) are required for tethering Pink1 mRNA to mitochondria via an RNA-binding domain in SYNJ2. This neuron-specific adaptation for local translation of PINK1 provides distal mitochondria with a continuous supply of PINK1 for activation of mitophagy.

\section{Introduction}

The large size of neurons and the need to maintain mitochondrial health far from the cell body contribute to the vulnerability of neurons to mitochondrial insults (Bolam and Pissadaki, 2012; Harbauer, 2017; Misgeld and Schwarz, 2017). Almost all mitochondrial proteins are encoded in the nucleus. Thus, the replacement and rejuvenation of mitochondria in distal axons and dendrites likely arises from mitochondrial transport in combination with mitochondrial fusion and fission and the mitophagic degradation of damaged organelles. However, there are 
limitations to the feasibility of transport as the only source of new mitochondrial proteins (Misgeld and Schwarz, 2017). Mitochondria travel at a speed of $\sim 0.5 \mu \mathrm{m} / \mathrm{s}$ (Misgeld and Schwarz, 2017) and therefore a mitochondrion generated in the cell body will take several days to reach the end of a $1 \mathrm{~m}$ long axon. This exceeds the lifetime of many short-lived mitochondrial proteins (Vincow et al., 2013). Likewise, proteins of the respiratory chain encoded by the mitochondrial genome are constantly synthesized in axonal and dendritic mitochondria (Yousefi et al., 2021), but how the complementary nuclear-encoded subunits are supplied is unknown. Local translation of mitochondrial mRNAs provides a possible resolution of this problem. Recent studies have characterized axonal mRNA species (Gumy et al., 2011; Shigeoka et al., 2016; Zivraj et al., 2010), and all such studies have detected a significant amount of nuclear-encoded mitochondrial transcripts in axons. Synaptic synthesis and protein import of mitochondrial proteins has been observed (Cioni et al., 2019; Kuzniewska et al., 2020) and global inhibition of protein synthesis in axons affects mitochondrial health (Hillefors et al., 2007). However, the mechanisms transporting nuclear-encoded mitochondrial mRNAs into neurites are largely unknown.

Failure to induce mitophagy has been linked to the etiology of Parkinson's disease (PD); two requirements for damage-induced mitophagy - Parkin and PTEN-induced kinase 1 (PINK1) are mutated in hereditary forms of PD (Exner et al., 2012). This pathway involves the constant, ongoing synthesis, mitochondrial import, and rapid degradation of PINK1 as long as mitochondria are healthy (Narendra et al., 2010). The half-life of PINK1 has been estimated to be in the order of minutes (Ando et al., 2017; Lazarou et al., 2012; Lin and Kang, 2008). Mitochondrial damage stops PINK1 import and the consequent stabilization of PINK1 triggers the translocation of Parkin to this organelle and initiates mitophagy (Durcan and Fon, 2015; Pickrell and Youle, 2015; Yamano et al., 2016). Evidence from our lab determined that acutely damaged mitochondria in axons can undergo local mitophagy and that this mitophagy required both PINK1 and Parkin (Ashrafi et al., 2014). For this mechanism to work in neurons, however, a constant supply of freshly synthesized PINK1 is required regardless of the distance of the mitochondrion from the cell body, thus raising the question of how a nuclear-encoded protein with a half-life of only minutes can be constantly synthesized far from the cell body. Therefore we selected Pink1 mRNA as a model to investigate the transport of RNA encoding mitochondrial proteins.

Using live-imaging of Pink1 mRNA, we observed co-transport of Pink1 mRNA with mitochondria and identified a tethering mechanism that uses the mitochondrial outer membrane protein Synaptojanin 2 binding protein (SYNJ2BP) and a neuron-specific and RNAbinding splice isoform of Synaptojanin 2 (SYNJ2). The neuron-specific co-transport of Pink1 mRNA with mitochondria enables dendritic and axonal translation of PINK1 to support the 
removal of damaged organelles and represents a neuron-specific adaptation of the PINK1/Parkin pathway.

\section{Results}

\section{Local translation is required for PINK1 activity in axons}

As the half-life of PINK1 in mammalian neurons may not be the same as that reported in cell lines, we used antibodies to human PINK1 to examine endogenous PINK1 stabilization and clearance in human iPSC-derived neurons (Figure 1A and B). PINK1 levels increased upon depolarization of mitochondria with the ionophore carbonyl cyanide m-chlorophenyl hydrazone (CCCP). This effect could be prevented by addition of the protein synthesis inhibitor cycloheximide $(\mathrm{CHX})$, as expected from the model that calls for continuous synthesis PINK1 in order to respond to mitochondrial damage (Narendra et al., 2010). Upon washout of CCCP, PINK1 levels sharply declined within $30 \mathrm{~min}$ to baseline levels, indicating that also in neurons continuous cleavage of PINK1 in polarized mitochondria maintains the protein at low abundance (Fig S1A). Rapid degradation of PINK1 in healthy mitochondria should prevent its effective transport in axons and dendrites. Instead, local translation of Pink1 mRNA could be the source of the PINK1 protein that supports local mitophagy (Ashrafi et al., 2014). Using a compartmentalized culture system and rodent embryonic hippocampal neurons (Fig. S1B), we asked whether the mitochondrial translocation of Parkin, which is directly dependent on PINK1 stabilization (Ashrafi et al., 2014; Vives-Bauza et al., 2010), occurred in the presence of CHX. $\mathrm{CHX}$ was applied to the axonal chamber four hours prior to induction of mitophagy with Antimycin A, using a concentration that has previously been shown to elicit PINK1-dependent mitophagy in axons (Ashrafi et al., 2014). Parkin translocation was visualized by transfection of YFP-tagged Parkin and mitochondrially targeted DsRed (mitoDsRed) (Fig. 1C). The percentage of Parkin-positive mitochondria was quantified before and after mitochondrial depolarization with Antimycin A. Approximately $10 \%$ of mitochondria were Parkin positive prior to Antimycin A (Fig. 1C and Ashrafi et al. 2014). Upon addition of Antimycin A, 29\% of mitochondria were Parkin-positive. Inhibition of protein synthesis by $\mathrm{CHX}$ eliminated $80 \%$ of this increase; only $13 \%$ of mitochondria accumulated Parkin (Fig. 1D). Thus local translation contributes substantially to efficient induction of mitophagy in axons.

Databases of axonal transcriptomes report Pink1 mRNA in axons (Shigeoka et al., 2016; Zivraj et al., 2010), which we confirmed by qPCR using hippocampal neurons cultured in microfluidic devices. RNA was extracted from the somal compartment, which represents a mix of neuron cell bodies, dendrites, and axons, as well as some non-neuronal cells, and compared it to RNA extracted from the axon-only compartment. The relative abundances of the Pink 1 transcript and a known cell-body restricted RNA, gamma actin, were determined and normalized to the amount of mitochondrial rRNA, which is transported inside mitochondria as part of 
mitochondrial trafficking and therefore not excluded from the axon. The gamma actin transcript was almost absent from the axonal fraction, whereas Pink1 mRNA was readily detected (Fig. $1 \mathrm{E}$ and $\mathrm{S} 1 \mathrm{C})$.

To establish whether Pink1 mRNA is also present in adult axons in vivo, we used adenoassociated virus injection into the eye to express transcripts for either a sequence-tagged Pink1 or GFP control transcript in mouse retinal ganglion cells, cells which project their axons to the brain exclusively through the optic nerve. Four weeks after the injection, RNA preparations from retina and from the optic nerve segment prior to the optic chiasm were analyzed by qPCR and the observed amounts normalized to the abundance of beta actin transcript to account for variances in the amount of tissue harvested. The exogenous Pink1 transcript was detectable in both tissues and had therefore been transported into the optic nerve, whereas the control GFP transcript was barely detectable in the axon (Fig. 1F). The selective presence of the expressed Pink1 mRNA relative to the GFP mRNA argues against simple diffusion as a mechanism of transport and corroborates our findings of axonal Pink1 mRNA in an adult in vivo model.

In order to test whether we could observe translation of PINK1 in axons, we used the photoconvertible protein Kaede and targeted it to mitochondria using either a generic mitotargeting domain (mito-Kaede, using the COX8a presequence, amino acids 1-36) or a portion of the PINK1 protein (PINK1-N-Kaede, amino acids 1-225). By repeated illumination with 405 $\mathrm{nm}$ laser light, Kaede is irreversibly converted into a red fluorophore, which allows the detection of newly synthesized, non-photoconverted and therefore green-fluorescent Kaede (RaabGraham et al., 2011). The N-terminus of PINK1 was sufficient to support local translation, as we were able to detect freshly synthesized, non-photoconverted signal for the PINK1-N-Kaede fusion protein 45 min after photoconversion in axonal mitochondria that were also positive for the photoconverted Kaede-red, whereas the signal for mito-Kaede did not recover (Fig. 1G and $\mathrm{H}$ ). This correlates with the sequences within the transcript that are necessary for transport of the Pink1 transcript as will be shown subsequently. The lack of recovery for mito-Kaede argues that a transcript not explicitly targeted to neurites could not supply the distal axons in this short time frame via protein transport from the soma, further strengthening the hypothesis that local translation supplies a substantial fraction of fresh PINK1 protein.

\section{Pink1 mRNA localizes to mitochondria in neurons}

The endogenous Pink1 transcript, as localized by RNAscope in situ hybridization, occurs in clusters along axons that are reminiscent of the distribution of mitochondria (Fig. 2A). Indeed, immunolabelling of the mitochondrial protein ATP5a in the same axons, revealed extensive co-localization of the two signals (Fig. 2A, left panel). Pink1 transcripts also localized to dendritic mitochondria (Fig. 2A right panel). With STED imaging, the Pink1 mRNA signal was 
resolved into multiple small puncta on each mitochondrion. In contrast, the control beta actin transcript did not overlap with axonal mitochondria (Fig. 2B).

To visualize transport of Pink1 mRNA in live neurons, we used the MS2/PP7-split-Venus approach for background-free mRNA imaging (Wu et al. 2014, Fig. 2C). This method combines the specific binding of the MS2 or PP7 viral coat proteins to their respective RNA stem loops with fluorophore reconstitution. Briefly, the mRNA of interest is expressed carrying a tag consisting of alternating MS2 and PP7 stem loops. In the same cell, the PP7- and MS2-binding coat proteins are expressed and each is fused to one half of split-Venus. Therefore, only in the presence of the transcript are the two halves of Venus in sufficient proximity to reconstitute a fluorescent protein (Fig. 2C). We therefore added 12 copies of PP7 and MS2 stem loops to a rat Pink 1 construct that included the relatively short 3' and 5' untranslated regions (UTR) of this gene. As the split-Venus approach contains the entire open reading frame (ORF), expression of this construct will lead to overexpression of the PINK1 protein. In order to avoid confounding effects of PINK1 overexpression, which for example, induces mitochondrial arrest (Wang et al., 2011), we also introduced a kinase dead mutation (K219M). The stem/looptagged Pink1 mRNA was readily detected in cell bodies and dendrites (Fig. S2A). However, detection of the transcript in axons (marked by AnkyrinG-mCherry) was limited using this method due to the difficulty of having adequate axonal levels of the fluorescently-tagged coat proteins present in axons (Fig. S2B and C). Nevertheless, small Pink1 mRNA puncta were observed in some proximal axons (Fig. 2D). Unlike the RNAscope data, in which every mitochondrion was associated with detectable Pink1 mRNA, the reconstituted Venus fluorescence was not detected on every mitochondrion, which likely represents the technical limit to the sensitivity of the method as applied to neurites.

Whether detected by RNAscope or with the MS2/PP7 split-Venus method, the Pink1 transcripts were on mitochondria (Fig. 2A, B and D). Using the Mander's correlation coefficient we quantified our imaging results and found a significant overlap between the Pink 1 mRNA and mitochondrial signals regardless of the method used (Fig. 2E). In order to exclude that the relative frequency of mitochondria in axons would produce the same overlap randomly, we flipped the mitochondrial channel of the straightened image and performed the same quantification. The mitochondrial localization of the Pink1 mRNA was significantly higher in the original images compared to the flipped control (Venus-flip, Fig. 2E).

As seen with RNAscope, Pink1 mRNA visualized with MS2/PP7 split-Venus also co-localized with dendritic mitochondria and to a somewhat lesser extent with mitochondria in the cell body. We observed a significantly higher overlap in all cases when comparing the Mander's correlation coefficients in neuronal compartments as compared to the flipped quantification (Fig 2F-G and S2D). 
Additionally, when beta actin mRNA was similarly tagged, small fluorescent puncta were observed, as expected for a transcript transported as a ribonucleoprotein particle (RNP). The beta actin transcripts did not co-localize with mitochondria in the cell body (Mander's coefficient was not significantly higher than the flipped quantification control), but we observed a marginal increase in colocalization in dendritic mitochondria. The slightly higher Mander's coefficient in dendrites is in line with observations that also non-mitochondrial transcripts including actin may use mitochondria as platforms for protein synthesis (Cioni et al., 2019; Spillane et al., 2013). Likewise, the beta actin mRNA has been found to localize to mitochondria in HeLa cells (Briley et al., 2015), supporting the physiological relevance of a closer association between mitochondria and this transcript.

Nonetheless, a significant difference remained between the two transcripts indicating a high likelihood that, unlike beta actin mRNA, Pink1 transcripts are actively localized to mitochondria (Fig. 2E-G). Additionally, we tested other mitochondrial transcripts. We chose two transcripts of MTS-containing proteins that have previously been reported to be either axonally localized, Cox4i (Kar et al., 2017), or mitochondrially localized, Atp5f1b (Margeot et al., 2002). Both transcripts are also detected in axonal transcript databases (Gumy et al., 2011; Shigeoka et al., 2016), yet encode proteins of very different half-lives (COX4i: approx. $30 \mathrm{~min}$, ATP5F1b: approx. six days) (Schwanhäusser et al., 2011). Both the full length Cox4i and the Atp5f1b transcripts (including their 3' and 5'UTRs) were significantly colocalized with mitochondria relative to the respective flipped-image control, albeit to a lesser extent compared to Pink1 (Fig. S4E and F). This is in line with the findings by Cohen et al. in the accompanying manuscript (Cohen et al., 2021), that also find extensive co-localization of mitochondrial transcripts with neuronal mitochondria.

As late endosomes had recently been implicated in the translation of mitochondrial transcripts in axons (Cioni et al., 2019), we also analyzed Pink1 mRNA localization in relation to endosomes labelled by mCherry-Rab7a. In the cell body the Pink1 transcript did not significantly overlap with late endosomes (Fig. S2G and $H$ ), whereas its association with mitochondria in the soma was clear. This colocalization in the soma together with the presence of the transcript on mitochondria in processes suggests that the Pink1 transcript is transported through its association with mitochondria rather than in separate RNPs.

\section{Pink1 mRNA is co-transported with mitochondria}

Because Pink1 mRNA was present on mitochondria in both axons and dendrites but live imaging of Pink1 particles in axons was limited, we analyzed the transport of the mRNA mainly in dendrites. In time-lapse images and kymographs, Pink1 mRNA was on both moving and stationary mitochondria (Fig. 3A; movie S1). Most Pink1 mRNA particles and their associated mitochondria were stationary, as expected from the predominance of stationary mitochondria 
in dendrites (Overly et al., 1996) (red arrowhead, Fig. 3A). Pink1 mRNA particles were also present on moving mitochondria (yellow arrowhead, Fig. 3A) and their movements mirrored those of the organelles, as indicated by their overlapping kymography traces (Fig 3A, lower panel). The Pink1 mRNA and mitochondria behaved as if bound together; the Pink1 mRNA stopped and started when its associated mitochondrion stopped and started. On a few occasions, we observed Pink1 mRNA particles that were not on a detectable mitochondrion. In some of these cases, kymographs suggested possible associations and dissociations from moving mitochondria (Fig 3B, S3A; movie S2). In most cases, however, the mRNA and mitochondrion remained together for the duration of the observation and this was true in both dendrites and axons (Fig. S3B; move S3). In 43 movies from dendrites we observed 96 moving mRNA particles, and in $89 \%$ of the cases Pink1 mRNA and mitochondria moved in synchrony (compare Fig. 3A-C; movies S1-3). In cases when we could not detect a corresponding mitochondrial trace the mRNA particles were stationary or moved only very short distances, while all long-range transport events happened in association with mitochondrial transport (Fig. 3B, C, S3; movie S2). There was no obvious directional preference of movement (time spent in motion for Pink1 mRNA particles: anterograde moving $3.13 \% \pm 0.53 \%$; retrograde moving $2.51 \% \pm 0.51 \% ; p=0.41$, Student's t-test, $n=29$ dendrites). This implies that mitochondrial transport of Pink1 mRNA is not directional and strictly for the delivery of this transcript from the soma to distal regions. Rather the mRNA movement mirrors the bidirectional movement of mitochondria (Overly et al., 1996). Together with the presence of the mRNA on the stationary pool of mitochondria, the bidirectional movement implies an ongoing association even in the periphery of the cells.

Overexpression of catalytically active PINK1 protein inhibits mitochondrial movement (Wang et al., 2011). We therefore compared the motility of the mRNA encoding wild-type (WT) and kinase-dead PINK1, to determine the consequences of the resulting overexpressed proteins. As expected from the extensive coupling of Pink1 mRNA transport to mitochondria, the transport of the mRNA encoding the catalytically active version was affected by the resulting overexpression of catalytically active PINK1 (Fig. 3D). On average, Pink1 mRNA particles spent $60 \%$ less time in motion, when the WT form of PINK1 protein was expressed compared to the expression of the kinase-dead mutant.

\section{Translation of the PINK1 mitochondrial targeting sequence is necessary but insufficient for Pink1 mRNA association with mitochondria}

To identify the mechanism that tethers the Pink1 transcript to the mitochondrial surface, we expressed shortened versions of the Pink1 transcript fused to the coding sequence for BFP (Fig. 4). The BFP sequence alone did not associate with mitochondria. We therefore asked whether any portions of the Pink 1 transcript could convey mitochondrial association onto a chimeric construct (Fig. 4A). We analyzed the extent of mitochondrial transcript colocalization 
in neuronal somata with the MS2/PP7-split-Venus system. Although many RNA-binding proteins (RBPs) bind within the untranslated regions (UTRs) of mRNAs (Holt and Schuman, 2013), neither of the Pink1 UTRs was sufficient for inducing the mitochondrial localization of the BFP transcript (Fig. 4A and S4A). Likewise, a transcript that included the C-terminal part of the PINK1 protein (nucleotides 676-1743 of the open reading frame (ORF) and the 3' UTR of Pink1) did not convey localization to mitochondria (Fig. 4A and S4A). However, inclusion of the N-terminal part of the PINK1 protein (the 5' UTR and nucleotides 1-675 of the Pink1 ORF) was sufficient to localize the mRNA to mitochondria (Pink1 5'UTR+N-BFP, Fig. 4A and B). This is consistent with our observation that fusion of the PINK1 N-terminus (amino acids 1-225, encoded by bp 1-675) supports local translation as measured by Kaede reappearance after photo-conversion (Fig. $1 \mathrm{G}$ and $\mathrm{H}$ ).

The Pink1 $5^{\prime} U T R+N-B F P$ construct contained both the start codon and the mitochondrial targeting sequence (MTS) of PINK1 and thereby suggested that translation of the transcript might be required for its mitochondrial localization. Indeed, expression of the full length Pink1 construct in the presence of the translation inhibitor puromycin altered the Pink1 mRNA distribution. After one hour incubation with $200 \mu \mathrm{g} / \mathrm{ml}$ puromycin, full length Pink1 mRNA had shifted from mitochondria to the cytosol (Fig. S4B, quantified in Fig. 4C). Puromycin dissociates both nascent chains and mRNA from ribosomes, thus translation is required for targeting the Pink1 mRNA to mitochondria. A construct lacking the start codon did not localize to mitochondria (Pink1 N $\triangle$ atg-BFP, Fig. $4 \mathrm{~A}$ and D), which further corroborated the requirement for translation. We still observed translation of the BFP portion of this transcript (Fig. 4D), presumably due to a downstream methionine.

The requirement for translation raised the possibility that the association of the mRNA with mitochondria was driven by the MTS on the nascent chain, similar to the targeting of ribosomes to the ER upon translation of ER proteins (Hegde and Bernstein, 2006). Although mitochondria can import proteins after their translation, co-translational import may occur as soon as the MTS leaves the ribosomal tunnel (Verner, 1993). The nascent MTS interacts with the receptors of the translocase of the outer membrane (TOM) complex (Harbauer et al., 2014), thereby driving the association of the ribosome and mRNA with the mitochondrial surface. This phenomenon has been observed in yeast and was recently also demonstrated in cultured mammalian cells (Fazal et al., 2019; Vardi-Oknin and Arava, 2019). We therefore hypothesized that the MTS of PINK1 might be sufficient to localize its mRNA to mitochondria. The first 34 amino acids of PINK1 are sufficient to drive protein import into mitochondria (Okatsu et al., 2015), and indeed when we expressed a construct consisting of the 5'UTR and MTS of Pink1 fused to BFP (Pink1 5'UTR+MTS-BFP), the BFP-fusion protein localized to mitochondria (Fig. 4E). This chimeric Pink1/BFP transcript, however, was still cytoplasmic (Fig. 4E). The interaction of the nascent chain with the TOM complex therefore is not sufficient to stabilize 
the transcript on mitochondria. The Mander's correlation coefficient was comparable for the Pink1 5'UTR+MTS-BFP and Pink1 NAatg-BFP constructs (Fig. 4A). As these two overlapping transcripts together include all the sequences present in the mitochondrially targeted Pink1 $5^{\prime} U T R+N-B F P$ construct, yet neither is sufficient to promote mitochondrial localization on its own, multiple sequences within the mRNA seem required for efficient mitochondrial localization. This prompted us to investigate the involvement of RBPs in Pink1 mRNA localization.

\section{SYNJ2BP knock down redistributes Pink1 mRNA into RNA granules and inhibits local mitophagy}

Overexpression of the mitochondrial outer membrane protein SYNJ2 Binding Protein (SYNJ2BP, also known as Outer Membrane Protein 25, OMP25) leads to an increase in mitoER contact sites that are enriched for ribosomes (Hung et al., 2017). A recent preprint now also connects SYNJ2BP with the localization of mitochondrial RNAs to the outer mitochondrial membrane (Qin et al., 2020).

We therefore knocked down SYNJ2BP expression in hippocampal neurons (Fig. S5A) and analyzed the localization of the Pink1 transcript using the MS2/PP7-split-Venus method. Knockdown of SYNJ2BP, but not control shRNA, diminished the interaction between Pink1 mRNA and mitochondria in the cell body and prevented the transport of the transcript into dendrites (Fig. 5A and B). This effect was specific to the loss of SYNJ2BP as expression of an shRNA-resistant SYNJ2BP rescued this effect (Fig 5B). The Pink1 mRNA left in the cell body formed larger aggregates that colocalized with RFP-DDX, an RNA helicase that is present in processing bodies (P-bodies, Fig. 5C and S5B). P-bodies are a cellular RNA storage compartments that sequester mostly untranslated mRNA and contain many RNA degrading enzymes (Sfakianos et al., 2016; Wilbertz et al., 2019). In contrast to its effect on Pink1 mRNA, the colocalization of beta actin mRNA and RFP-DDX6 was unchanged by SYNJ2BP knock down (Fig. S5C and D). These results indicate that loss of SYNJ2BP has a selective effect on Pink1 mRNA localization.

As RNA localization to P-bodies may be linked to its degradation we analyzed the levels of Pink1 mRNA upon SYNJ2BP knockdown but did not find a decrease, but rather a trend towards an increase in the amount of Pink1 transcript (Fig. S5E), which may be due to compensatory mechanisms upregulating Pink1 expression. Also, SYNJ2BP knockdown did not prevent expression of an mRNA that would otherwise be targeted to mitochondria; we still observed translation of the PINK1-BFP chimeric construct (Fig. S5F) in the cell body.

The requirement for SYNJ2BP for Pink1 mRNA association with mitochondria and cotransport predicted that knockdown of SYNJ2BP would prevent local activation of axonal mitophagy, in keeping with the dependence of the PINK1/Parkin pathway on local axonal translation (Fig. 1). 
To measure mitophagy when SYNJ2BP is knocked down, we turned to the mitochondrially localized, pH-sensitive reporter mito-mKeima (Katayama et al., 2011), a fluorophore with a bimodal excitation spectrum depending on protonation of its chromophore. At neutral $\mathrm{pH}$, when mitochondria are healthy, Keima is most effectively excited at $442 \mathrm{~nm}$ (represented in green), whereas under acidic conditions, upon induction of mitophagy and fusion with lysosomes, Keima excitation peaks at $550 \mathrm{~nm}$ (pseudocolored red). Consistent with the need for Pink1 mRNA transport, knockdown of SYNJ2BP reduced the incidence of Antimycin A-induced mitophagy in distal axons (Fig. 5D and E), confirming the local loss of PINK1 function.

\section{Pink1 mRNA mitochondrial localization is neuron-specific and depends on SYNJ2a}

Pink1 mRNA was not on mitochondria in COS-7 cells (Fig. 6A); the mitochondrial association of the transcript may therefore be neuron-specific. The lack of mitochondrial localization of the transcript allowed us to ask what missing neuronal component was needed to bring the Pink1 mRNA to COS-7 mitochondria. As SYNJ2BP is ubiquitously expressed, we analyzed if its interaction partners, the SYNJ2a splice variant of Synaptojanin 2 (SYNJ2) (Nemoto and De Camilli, 1999) or the ER protein Ribosome Binding Protein 1 (RRBP1)(Hung et al., 2017) are enriched in neurons over fibroblasts. Synj2a transcripts were five-fold higher in hippocampal cultures than in fibroblasts (Fig. 6B), unlike the Rrbp1 transcripts levels, which were seven-fold higher in fibroblasts than neurons (Fig. 6C).

We therefore overexpressed SYNJ2a and SYNJ2BP in COS-7 cells and found that Pink1 mRNA was thereby relocalized to mitochondria (Fig. 6D and E). In contrast, overexpression of the related protein Synaptojanin 1 (SYNJ1) together with SYNJ2BP did not change the cytoplasmic localization of the Pink1 transcript. The effect of SYNJ2a overexpression was specific for Pink1 mRNA; beta actin mRNA remained cytosolic (Fig. 6D and E). As had been reported in Nemoto and De Camilli (1999) overexpression of SYNJ2BP and SYNJ2a also led to mitochondrial clustering (compare Fig. 6E).

Given our findings in COS-7 cells, we tested the importance of SYNJ2 for the mitochondrial localization of Pink1 mRNA in neurons. Localization of Pink1 mRNA with neuronal mitochondria was diminished by knock down of SYNJ2, but rescued by co-expression of an shRNA-resistant SYNJ2 construct (Fig. S6A and B). We therefore conclude that in addition to co-translational targeting, SYNJ2 acts as a mitochondrial anchor for Pink1 mRNA.

\section{RNA-binding by SYNJ2 is necessary for Pink1 mRNA localization to mitochondria}

SYNJ2 is an inositol 5'-phosphatase with an established role in endocytosis (Nemoto et al., 1997). It is, however, also predicted to contain an RNA recognition motif (RRM) domain (Hsu and Mao, 2015), although its ability to bind nucleotides has not been established. Based on the homology between different RRM domains (Maris et al., 2005), we identified the residues Valine 909, Glutamine 951 and Leucine 953 (VQL) in SYNJ2 as likely to be involved in RNA binding. We expressed the myc-tagged RRM domain of rat SYNJ2 in HEK cells and used 254 
nm UV-Crosslinking and Immuno-Precipitation (CLIP) to determine if the RRM domain could be crosslinked to RNA (Castello et al., 2012; Greenberg, 1979). Upon UV irradiation, we observed several crosslinked species of WT SYNJ2 RRM running at higher molecular weights, as expected for an RNA-binding domain (Fig. 7A). Mutation of the VQL residues to Alanine abolished its RNA-binding ability and prevented the appearance of the cross-linked species (VQL/AAA, Fig. 7A lane 3).

To determine if the RNA-binding property of SYNJ2a was sufficient to target Pink1 mRNA to mitochondria, we constructed both a WT and a VQL/AAA version of an artificial tether analogous to the constructs used in Nemoto et al., 1997. By directly fusing the open reading frame of SYNJ2a to the transmembrane domain of SYNJ2BP (SYNJ2mito), the protein will be targeted to mitochondria even in the absence of the endogenous SYNJ2BP. Indeed, the overexpression of SYNJ2mito WT in neurons (Fig. S7A) was sufficient to overcome the knock down of SYNJ2BP and relocalized Pink1 mRNA back onto mitochondria (Fig. 7B and C). This was again specific to Pink1, as the localization of beta actin transcripts was not altered (Fig. S7B and C). Upon expression of VQL/AAA mutant of the SYNJ2mito construct, the Pink1 transcript remained cytosolic (Fig. 7B and C). The RNA-binding capacity of SYNJ2 can therefore mediate the localization of Pink1 mRNA to mitochondria.

Since we have observed that translation is crucial in the Pink1 mRNA recruitment to mitochondria, we tested whether active translation is necessary for SYNJ2 to localize to the mitochondrial surface. Using a proximity-ligation assay (PLA) SYNJ2 and SYNJ2BP were observed to be closely associated, but upon addition of Puromycin this interaction was reduced to background levels when using mitochondrial proteins outside the RNA adapter complex (PLA of SYNJ2 with RHOT1 and PLA of SYNJ2BP and ATP5b) (Fig. 7D and E). This suggests that in the absence of translation SYNJ2 may be cytosolic, as is the Pink1 mRNA (compare Fig. 4C), but upon translation of PINK1, SYNJ2 and SYNJ2BP can interact and tether the Pink1 mRNA to mitochondria.

\section{Discussion}

Our study identifies a neuron-specific mechanism for localizing Pink1 mRNA to mitochondria and that thereby facilitates the transport of Pink1 mRNA into axons and dendrites. Application of acute stressors to axons triggers local PINK1-dependent mitophagy (Ashrafi et al., 2014), yet the short half-life of the PINK1 protein makes its transport as a protein into distant regions of the cell highly unlikely. The PINK1/Parkin pathway depends on the ongoing synthesis of PINK1 and is activated by the arrest of PINK1 degradation. How then is the PINK1 protein steadily supplied to distal axons and dendrites? Our finding of a transport mechanism for Pink1 mRNA and of the dependence of axonal mitophagy on axonal translation establishes a mechanism for local PINK1 translation that can fulfill the requirement for local PINK1 supply. 
The failure of local mechanisms for quality control and clearance of mitochondria has been invoked in PD to account for axon degeneration prior to cell death (Cheng et al., 2010; Sliter et al., 2018). Activation of the PINK1/Parkin pathway likely contributes to axonal quality control as it causes acutely depolarized mitochondria to arrest their movement and recruit autophagic and lysosomal markers (Ashrafi et al., 2014; Hsieh et al., 2016). This pathway likely acts in parallel with other forms of mitochondrial clearance from axons. Alternative mechanisms may include the retrograde transport of damaged organelles towards the cell body (Evans and Holzbaur, 2020; Lee et al., 2018; Lin et al., 2017; McWilliams et al., 2018; Miller and Sheetz, 2004). The relative contributions of these mechanisms may vary with the nature of mitochondrial damage and with age; an age-dependent increase in the amount of PINK1dependent mitophagy occurs in Drosophila (Cornelissen et al., 2018) that had not been detected in earlier studies (Lee et al., 2018; McWilliams et al., 2018). Mitochondrial transport has already been known to be crucial for neuronal health by rejuvenating the protein pool of axonal and dendritic mitochondria (Misgeld and Schwarz, 2017). In light of its role in the transport of Pink1 mRNA and consequently for local mitophagy, mitochondrial transport is therefore important on more than one level for sustaining healthy mitostasis.

Local translation in dendrites is well established (Donnelly et al., 2010; Holt and Schuman, 2013) but axonal translation in vivo was controversial. Recent studies, however, demonstrated the presence of translating ribosomes in axons in vivo, regardless of the age of the animal (Biever et al., 2020; Ostroff et al., 2019; Shigeoka et al., 2016). All studies report Pink1 mRNA among the axonally translated transcripts, which is consistent with our findings of Pink1 transcripts in axons of cultured neurons and optic nerve, whether by qPCR (Fig. 1E and F), in situ hybridization (RNAscope, Fig. 2A), or the MS2/PP7-split-Venus approach (Fig. 2D-E). We also detect the local synthesis of a PINK1-N-Kaede construct in axons (Fig 1G and H). Moreover, because inhibition of translation specifically in axons prevented Parkin recruitment to damaged mitochondria (Fig.1D), we have established a functional significance for its axonal translation.

Axonal transcripts have been found to regulate axon growth, maintenance and regeneration after injury (Andreassi et al., 2010; Ben-Yaakov et al., 2012; Hanz et al., 2003; Hengst et al., 2009; Sotelo-Silveira et al., 2008; Terenzio et al., 2018; Verma et al., 2005; Yoon et al., 2012). In addition, mitochondria have come into the spotlight as centers for axonal translation (Cioni et al., 2019; Cosker et al., 2016; Spillane et al., 2013). However, to the extent these transcripts have been localized and their transport mechanisms examined, these transcripts are thought to travel as independent RNP granules. Pink1 mRNA, however, did not form such separate RNP granules. Instead, Pink1 mRNA consistently colocalized with mitochondria and thus offers a different model for a means of transport. We observed all processive movements of Pink1 mRNA to be coupled with mitochondrial movements (Fig. 3C) and measures that are 
known to alter mitochondrial motility, like the overexpression of kinase-active PINK1 protein, were reflected in the motility of the Pink1 mRNA (Fig. 3D). When transcripts were observed without a detectable associated mitochondrion, they were either stationary or engaged in only short-range and often erratic movements (Fig. 3B). Sensitive detection of Pink1 mRNA by RNAscope found the transcript on almost all mitochondria in axons and dendrites (Fig. 2A and $B$ ), which would include those that were stationary or moving retrograde before fixation. Thus, the association of transcript and mitochondrion is probably not solely a mechanism for delivery from the cell body to the periphery, but may also facilitate the constant translation of PINK1 as is necessitated by its role in mitochondrial quality control (Narendra et al., 2010).

The localization of the transcript to neuronal mitochondria was verified using both RNAscope and a live imaging approach using split-Venus fluorophore reconstitution on a stem/loop tagged Pink1 construct. RNAscope has the clear advantage of detecting the endogenous transcript, and was more easily applied to distal axons. However, it was not appropriate for live imaging of mRNA transcript. For this, we turned to the MS2/PP7-split-Venus method (Wu et al., 2014). Although it easily visualized transcripts in the soma and dendrites, detection of the Pink1 mRNA was challenging in axons. Split fluorophore reconstitution prevents detectable background from the unassembled components. It adds, however, to the complexity of the system, as the loss of any one of the ternary complex partners during transport leads to loss of signal. Genomic integration of the stem loops, as was done for beta actin in a transgenic mouse model (Lionnet et al., 2011), would permit imaging of endogenous transcripts, but would still face the need for two well-transported fluorophore constructs (compare Fig S2C). Transient overexpression, nevertheless, allowed the efficient screening of constructs in order to find cis-acting sequences in the Pink1 mRNA (Figure 4). Because the Pink1 mRNA was mitochondrial in all neuronal compartments, we were able to use transport in dendrites and mitochondrial colocalization in somata for defining how Pink1 mRNA localized to mitochondria and entered neuronal processes.

We examined the mechanism of the association between Pink1 mRNA and mitochondria and their co-transport by determining both the necessary cis-elements and requisite trans-acting factors. We determined that there are at least two sequences required for the mitochondrial localization of the transcript. Deletion of the start codon or inhibition of translation by puromycin indicated that translation of the PINK1 protein is one requirement for mitochondrial localization of the transcript (Fig. 4C and D). The N-terminal MTS of PINK1 may interact with the receptors of the TOM complex as soon as the nascent chain emerges from the ribosome and thereby confer mitochondrial localization to the transcript. But, though translation of PINK1 is necessary, translation of the MTS is not sufficient: a transcript in which this MTS properly directed its BFP-tagged translation product into mitochondria (Pink1 5'UTR+MTS-BFP, Fig. $4 \mathrm{E})$ remained cytosolic. Therefore, at least one additional sequence must be necessary and a 
comparison of the constructs in Figure 4 implies that it is likely found between base pairs 103 and 625 of the Pink1 open reading frame (compare Pink1 5 'UTR+N-BFP and Pink1 5'UTR+MTS BFP, Fig. 4A and B). If the Pink1 5UTR'+MTS-BFP transcript is transiently associated with the mitochondrion while BFP is being translated and translocated into the matrix, it does not remain associated with mitochondria as does full length Pink1 mRNA. In yeast, interaction between the nascent chain and mitochondria mediates the co-translational targeting of the Atp2p mRNA to the mitochondrial surface (Garcia et al., 2010), but there is an additional role of its 3'UTR for its mitochondrial localization (Margeot et al., 2002). This dual requirement is reminiscent of the bipartite targeting signal of Pink1 mRNA.

In exploring the trans-acting factors required in neurons, we discovered that knock down of the mitochondrial outer membrane protein SYNJ2BP is sufficient to remove Pink1 mRNA from mitochondria (Fig. 5A and B). Once released from mitochondria, Pink1 mRNA was sequestered in cytoplasmic granules containing the RNA helicase DDX6 (Fig. 5C and S5B). This sequestration did not represent a uniform change in cellular mRNA; beta actin mRNA distribution was unaltered (Fig. S5C and D). Thus the relocalization of the Pink1 transcript was likely to be a direct consequence of its inability to associate with mitochondria. SYNJ2BP itself has been suggested to have RNA-binding capabilities (Qin et al., 2020), but our observation that the mitochondrial localization of the Pink1 transcript was neuron-specific led us to explore the expression patterns of its interactors. Unlike the SYNJ2BP-interacting ER protein RRBP1 (Hung et al., 2017), which was much more abundant in fibroblasts (Fig. 6C), the mitochondriaspecific splice-variant of SYNJ2, SYNJ2a, was five-fold higher expressed in neurons over fibroblasts (Fig. 6B). Overexpression of Synj2a and SYNJ2BP was sufficient to target Pink1 mRNA to mitochondria in COS-7 cells (Fig6 D and E). PINK1 has been reported to alter proteins important for mitochondrial dynamics and ultrastructure (Chen and Dorn, 2013; Tsai et al., 2018; Wang et al., 2011); altered PINK1 biogenesis may therefore account for the clustering of mitochondria seen by us and others upon Synj2a overexpression (Nemoto and De Camilli, 1999). Alternatively the clustering may arise from other, yet to be identified substrates of the SYNJ2a/SYNJ2BP RNA tethering complex, or lipid alterations due to the inositol 5'-phosphatase activity of SYNJ2.

SYNJ2 is best known for its inositol 5'-phosphatase function during endocytosis, but we demonstrated an RNA-binding capacity of SYNJ2 (Fig. 7A) and showed that the engineered presence of this protein on the mitochondrial surface was sufficient to localize Pink1 mRNA at mitochondria, even in the absence of SYNJ2BP (Fig. 7B and C). Thus the only essential function of SYNJ2BP for the localization of the mRNA is its ability to recruit SYNJ2. Mutations that abolished RNA binding by the RNA-Recognition Motif in SYNJ2 demonstrated that it is indeed this capacity of SYNJ2 that is needed. It is interesting to speculate whether 
mitochondrially localized SYNJ2a would still be able to interact with its endosomal binding partners, thereby forming a mito-Endosome contact site. Fittingly, local translation in axons has been reported to occur at such interfaces (Cioni et al., 2019).

The mRNA tether we propose would thus entail SYNJ2BP anchored in the outer membrane and associating with its partner SYNJ2, which in turn holds the mRNA. SYNJ2a and SYNJ2BP were shown to directly interact in yeast two-hybrid screens and in purified proteins by virtue of a PDZ domain in SYNJ2BP and an interacting motif in SYNJ2a (Nemoto and De Camilli, 1999). We have confirmed that the proteins are associated in situ by PLA, however the association was greatly diminished when translation was inhibited (Fig. 7E). This translation-dependence is likely significant for the translation-dependent localization of Pink1 mRNA to neuronal mitochondria. Thus localization appears to involve two mechanisms acting in parallel and reinforcing one another. One model that combines these components would first envision active translation of the PINK1 MTS leading to co-translational protein import of the nascent chain and hence the relocation of the ribosome and associated Pink1 mRNA to the mitochondrial surface. Thereafter, the interaction of the mRNA and the mitochondrion is further stabilized through the SYNJ2/SYNJ2BP tether. This mechanism allows the Pink1 transcript to move together with mitochondria in neurons while providing a constant local source for freshly synthesized PINK1 protein.

Our observation also raises the question as to whether the predicted RRM domains of all Synaptojanins are also capable of RNA-binding. Beta actin mRNA was not redistributed upon expression of SYNJ2mito (Fig. S7B and C) and SYNJ1, while containing an RRM, could not replace SYNJ2 for localization of Pink1 mRNA (Fig. 6D and E). Thus, there is a substrate specificity to the RNA-binding capacity of the synaptojanins.

It will now be critical to determine what other mRNAs may be bound by SYNJ2. Mitochondrial transcripts are among the most abundant RNAs found in axons, and at least some mitochondrial proteins have a comparatively short half-life that may require their local translation to ensure mitostasis in axons (Harbauer, 2017; Misgeld and Schwarz, 2017). Both Atp5f1b and Cox4i transcripts were enriched on neuronal mitochondria (Fig. S2E and F), as well as Cox7c identified in the accompanying manuscript (Cohen et al., 2021), which makes them likely candidates for binding to SYNJ2a. Although loss of Pink1 does not compromise the viability of cultured neurons (Kitada et al., 2007), neuronal health deteriorated upon knock down of SYNJ2BP; this effect may indicate that there are other transcripts, vital to neurons, that depend on this tether for localization and transport.

PD primarily affects neurons. Our observations that mitochondrial localization of the Pink1 mRNA may be specific to neuronal cells (Fig. 6A) and that local translation and mitochondrial tethering through SYNJ2BP is required for the full activation of the PINK1/Parkin pathway in axons (Fig. 1D and 5E) point to the exceptional challenges that are faced by neurons as they 
seek to maintain healthy mitochondria in distal regions of their arbors. Effective transport of mRNA for mitochondrial proteins, whether on moving mitochondria or as RNP granules, likely contributes substantially to preserving neurites from degeneration.

\section{Acknowledgments}

We are grateful for plasmids shared with us by P. de Camilli (Yale University; FLAG-SYNJ2a and SYNJ1-FLAG). We thank L. Mkhitaryan and J. Lindner for technical support and the members of the T.L. Schwarz laboratory for their support and many fruitful discussions. We are grateful to S. Vasquez and M. Sahin's laboratory for assistance with primary hippocampal cultures; D. Tom, L. Ding, M. Ocaña and A. Begue from Harvard NeuroDiscovery Center's/Neurobiology Imaging Facility Enhanced Neuroimaging Core (NINDS P30 Core Center grant no. NS072030), as well as R. Kasper and M. Spitaler from the Imaging facilities of the MPIs for Neurobiology and Biochemistry, respectively, for assistance with live-cell imaging. This work was supported by grants from the National Institute of Health R01NS107490 and R01GM069808 to TLS, and P30HD018655 to the IDDRC Cellular Imaging and Molecular Genetics Cores. ABH was supported by a Howard Hughes Medical Institute fellowship from the Jane Coffin Childs Memorial Fund for Medical Research. Since 2019, she is an independent research group leader and a Rudolf-Mößbauer tenure track professor at Technical University of Munich Institute for Advanced Study and the Institute of Neuronal Cell Biology. In this role she is supported by the Max Planck Society and the Deutsche Forschungsgemeinschaft (DFG, German Research Foundation, HA 7728/2-1 and under Germany's Excellence Strategy within the framework of the Munich Cluster for Systems Neurology (EXC 2145 SyNergy - ID 390857198).

\section{Author Contributions}

$\mathrm{ABH}$ and TLS conceived of the project and wrote the manuscript. $\mathrm{ABH}$ designed and conducted most of the experiments. SW was responsible for RNAscope and superresolution imaging and the PINK1-Kaede imaging. JTH was responsible for imaging Atp5f1b and Cox4i live cell imaging and PLA experiments. WG contributed the analysis of PINK1 stability in human iPSC-derived neurons. GA conducted initial experiments for Parkin translocation in the presence of $\mathrm{CHX}, \mathrm{MO}$ conducted $\mathrm{qPCR}$ experiments and $\mathrm{ZC}$ assisted with $\mathrm{COS}$ cell experiments and cloning. RC, CW and $\mathrm{ZH}$ were responsible for expression of PINK1 constructs in mouse retinal ganglion cells.

\section{Declaration of Interests}

The authors declare no competing interests. 


\section{Figure Legends}

Figure 1. Local translation is required for PINK1 activity in axons (A) Representative Western blot showing the stabilization of PINK1 in response to the uncoupler CCCP $(20 \mu \mathrm{M}$ $2 \mathrm{~h}$ ) in human iPSC-derived cortical neurons, which can be prevented by addition of $5 \mu \mathrm{M}$ Cycloheximide (CHX) 20min prior to CCCP. (B) Quantification of PINK1 stabilization as in (A) normalized to GAPDH signal. Data is shown as mean \pm SEM; ANOVA with Tukey's multiple comparisons test, $n=6$ biological repeats per condition (C) Neurons grown in a compartmentalized culture chamber transfected with YFP-Parkin and a mitochondrial marker were treated with $40 \mu \mathrm{M}$ Antimycin A. The recruitment of Parkin to mitochondria (yellow arrowheads) was monitored with live cell imaging prior (start) and after 20 min addition of Antimycin A. (D) Quantification of Parkin-positive mitochondria in the axonal compartment before and after Antimycin A treatment in presence or absence of $35 \mu \mathrm{M}$ Cycloheximide. Data is shown as mean $\pm S E M$; Student's t-test, $n=3$ biological repeats per condition. (E) RNA isolated from cell body or axonal compartments of microfluidic devices was analyzed by qPCR. The abundance of the transcripts was normalized to mitochondrial rRNA. Data are shown on a log scale as mean \pm SEM; Student's t-test; $n=3$ independent microfluidic devices. (F) After intraorbital injection of AAV encoding either GFP or tagged PINK1 transcripts, retinal and optic nerve RNA was collected and the abundance of the exogenous transcripts analyzed by qPCR and normalized to beta Actin. Unlike GFP mRNA, Pink1 mRNA is actively transported into axons. Data are shown on a log scale as mean \pm SEM; Student's t-test, $n=4$ retina/optic nerve pairs. (G) Representative images and $(H)$ quantification of the photo-convertible fluorescent protein Kaede fused to PINK1-N (Amino acids 1-225, PINK1-N-Kaede) or Cox8 (Amino acids 1-36, mito-Kaede). The mitochondrial mean signal intensity of the non-photoconverted Kaede was quantified and is shown in the graph. Note that the intensity returning after 45 min post photoconversion is significantly higher in the PINK1-N-Kaede group. Student's t-test, $n=3-5$ axons. $\mathrm{p}<0.01^{\left({ }^{*}\right)}$ and $\mathrm{p}<0.0001^{\left({ }^{* * *}\right)}$. Scale bars $=10 \mu \mathrm{m}$. For further verification of microfluidic devices see Figure S1.

Figure 2. Pink1 mRNA localizes to mitochondria in neurons. (A) RNAscope in situ hybridization reveals Pink1 mRNA localization to mitochondria in axons and dendrites. Scale bar $=10 \mu \mathrm{m}$. (B) Representative superresolution STED images for endogenous Pink1 and beta actin RNA by in situ hybridization (RNAscope) and mitochondria detected by immunostaining for ATP5b. Scale bar $=2 \mu \mathrm{m}$. (C) Schematic of MS2/PP7-split-Venus method for mRNA imaging. (D-G) Live cell imaging of Pink1 and beta actin RNA in hippocampal neurons using the MS2/PP7-split-Venus method. (D) Representative image of co-localization of the tagged RNA with mitochondrially targeted BFP (mitoBFP). Scale bar $=10 \mu \mathrm{m}$. (E) Mander's correlation coefficient for RNA and mitochondrial channels. "Venus-flip" indicates that the mitochondrial 
channel, after digital straightening of the axon, had been flipped horizontally before quantification. Student's t-test, $n=10$ axons; $p<0.0001{ }^{\left({ }^{* * *}\right)}$. $(F$ and $G$ ) Mander's correlation coefficient analysis between RNA and mitochondrial channels in cell bodies and dendrites. "flipped control" indicates that the mitochondrial channel, after digital straightening in the case of dendrites, had been flipped horizontally before quantification Student's t-test, $n=39-43$ cell bodies, $16-20$ dendrites; $\left.p<0.0001^{* * * *}\right)$. Scale bar $=10 \mu \mathrm{m}$. For representative images, colocalization with endosomes and further detail on the MS2/PP7-split-Venus method see Figure S2.

Figure 3. Pink1 mRNA is co-transported with mitochondria. (A) Still-images and kymograph of a time-lapse movie from a dendrite showing Pink1 mRNA co-transported with a mitochondrion (yellow arrowhead) as well as stationary mitochondria associated with a Pink1 mRNA particle (red arrowhead). Scale bar $=5 \mu \mathrm{m}$ (B) Kymograph in which Pink1 mRNA appeared to transiently occur without an associated mitochondrion. While most mRNA particles were associated with mitochondria, including during transport (yellow arrowhead), particles without a mitochondrion were occasionally seen and might undergo short-range independent movement (blue and white arrowheads). A potential interpretation of this kymograph as indicating association and dissociation of the Pink1 mRNA from moving mitochondria is schematized in Figure S3. Scale bar $=10 \mu \mathrm{m}$. (C) Histogram depicting frequency of observed movements of 96 moving Pink1 mRNA particles from 46 dendrites over the indicated distances. (D) Overexpression of wild-type (WT) PINK1 protein decreases Pink1 mRNA motility relative to kinase-dead PINK1 protein. Average time spent in motion per dendrite was analyzed in $n=26-29$ dendrites from three independent experiments. Student's t-test, $\left.p<0.01{ }^{* *}\right)$.

Figure 4. Translation of the PINK1 mitochondrial targeting sequence is necessary but insufficient for Pink1 mRNA association with mitochondria

(A) Schematic representation of constructs used and Mander's correlation coefficients between the indicated Pink1/BFP chimeric constructs and mitochondria in hippocampal cell bodies. The distribution of full length Pink1 was repeated from Figure $2 \mathrm{~F}$ for comparison. Oneway ANOVA with Bonferroni post-hoc test; $n=28-41$ cell bodies, $\left.p<0.001{ }^{* * *}\right), p>0.05$ (\#). (B) Representative images for Pink1 5'UTR+N-BFP. (C) Mander's correlation coefficient between Pink1 transcript (kinase dead) and mitochondria in the presence or absence of puromycin. Student's t-test, $\mathrm{n}=26-28$ cell bodies, $\left.\mathrm{p}<0.001{ }^{(* \star}\right)$. (D) Representative images of Pink1- $N$ $\triangle$ atg-BFP RNA and protein. (E) Representative images for Pink1 5'UTR+MTS-BFP. Please note that the Pink1 5'UTR+MTS-BFP mRNA is largely cytosolic, although the encoded BFP 
protein localizes exclusively to mitochondria. Scale bars $=10 \mu \mathrm{m}$. For representative images see Figure S4.

Figure 5. SYNJ2BP knock down redistributes Pink1 mRNA to RNA granules and inhibits local mitophagy. (A) Neurons were treated with either control or SYNJ2BP shRNA for imaging of Pink 1 transcripts (kinase dead) by the split-Venus method and mitochondria. Representative images from the cell bodies and dendrites are shown. (B) Colocalization in the cell body as quantified with Mander's correlation coefficient. Coexpression of an shRNAresistant SYNJ2BP restored localization of Pink1 transcripts to mitochondria. Student's t-test, $\mathrm{n}=23-31$ cell bodies, $\left.\mathrm{p}<0.0001^{(* * *}\right)$. (C) Mander's correlation coefficient for colocalization in cell bodies of Pink1 transcript and P-bodies marked with RFP-DDX. Student's t-test, $n=16-20$ cell bodies, $\mathrm{p}<0.0001{ }^{(* * *)}$. (D) Representative images of the $\mathrm{pH}$-sensitive fluorophore mKeima targeted to mitochondria in axons from neurons also expressing either Control or SYNJ2BP shRNA, with and without Antimycin A treatment. (E) Mitophagy index for neurons treated as in (D). Student's t-test, $n=9-10$ axonal field of views, $\left.p<0.01{ }^{* *}\right)$. Scale bars $=10$ $\mu \mathrm{m}$. For the localization of beta actin mRNA and validation of the shRNA please refer to Figure S5.

Figure 6. Pink1 mRNA localization to mitochondria is neuron specific and depends on SYNJ2a. (A) Pink1 transcript is not localized to mitochondria in COS-7 cells. (B) qRT-PCR from primary fibroblasts and hippocampal neurons comparing the expression levels of the Synj2a splice variant. Data are shown as mean \pm SEM; Student's t-test; $n=3-5$ cultures. (C) qRT-PCR from primary fibroblasts and hippocampal neurons comparing the expression levels of the Rrbp1 transcript. Data are shown as mean \pm SEM; Student's t-test; $n=3$ cultures. (D) Mander's Coefficients of Pink1 and beta actin RNA colocalization with mitochondria in COS-7 cells overexpressing the indicated proteins. Data are shown as mean \pm SEM; Student's t-test; $\mathrm{n} \geq 3$ experiments scoring $\geq 30$ cells per condition total. $\left.\mathrm{p}<0.01{ }^{* *}\right)$. (E) Representative images of Pink1 and beta actin transcript localization in COS-7 cells with overexpression of the indicated proteins. Scale bars $=10 \mu \mathrm{m}$. For knock down of SYNJ2 in neurons, please refer to Figure S6.

Figure 7. RNA-binding by SYNJ2a is necessary for Pink1 mRNA localization to mitochondria. (A) Myc-tagged SYNJ2-RRM constructs were expressed in HEK 293T cells and irradiated as indicated with $254 \mathrm{~nm}$ UV light. Lysates were immunoprecipitated with antimyc and a representative anti-myc Western blot is shown. (B) Representative images of neuron cell bodies expressing SYNJ2BP shRNA and either WT or VQL/AAA SYNJ2mito, in 
addition to the split-Venus reporter for Pink1 mRNA and mitoRasp. Scale bar $=10 \mu \mathrm{m}$. (C) Colocalization as quantified with Mander's correlation coefficient between mitochondria and Pink1 (kinase dead) transcripts for cells as in (B). Student's t-test, $n=20-23$ cell bodies; $p<0.01$ $\left.\left({ }^{* *}\right), p<0.0001{ }^{* * * *}\right)$. (D) Representative images displaying the proximity ligation assay (PLA) using the indicated antibodies in the presence or absence of puromycin. (E) Quantification of PLA results as in (D) Student's t-test; $n \geq 3-5$ experiments scoring $\geq 15$ cells per condition total. $\left.\mathrm{p}<0.0001^{* * * *}\right)$. Data are shown as mean $\pm \mathrm{SEM}$; scale bars $=10 \mu \mathrm{m}$. For beta actin imaging in the presence of either WT or VQL/AAA SYNJ2mito in SYNJ2BP shRNA treated neurons see Figure S7.

Figure S1 corresponding to Figure 1. Microfluidic chambers exclude dendrites and lack detectable cell body transcript. (A) Quantification of PINK1 stabilization in human iPSCdervied cortical neurons upon CCCP treatment $(4.5 \mathrm{~h} 20 \mu \mathrm{M})$ and $30 \mathrm{~min}$ after washout of CCCP. Intensity is shown normalized to GAPDH signal. Data is shown as mean \pm SEM; ANOVA with Tukey's multiple comparisons test, $n=6$ biological repeats per condition (B) Immunolabeling of neurons grown in a microfluidic chamber for a dendritic (MAP2) and a neuronal (beta-III tubulin) marker. (C) qPCR analysis of RNA levels in the cell body and axonal compartment. Please note that the total amount of RNA isolated from the axonal side is approximately 20 times lower than the cell body. The same data normalized to mitochondrial rRNA is presented in Figure 1E.

Figure S2 corresponding to Fig 2. Analysis of the Pink1, Cox4i and Atp5f1b mRNA localization using the MS2/PP7/-split-Venus method. (A) Representative image for kinasedead Pink1 mRNA localization at mitochondria in all neuronal compartments using MS2/PP7split-Venus to visualize Pink1 transcripts, mitochondrially targeted BFP (mitoBFP) to visualize mitochondria and AnkyrinG-mCherry to identify the axon. (B and C) Representative image and quantification of the splitVenus subunits detected by their HA-tag and an iRFP cell-fill. Axons were traced as many fields of view as possible using the iRFP cell-fill and the mean intensity after background correction was used to calculate the ratios presented in $(C)$. The magenta line displays the mean ratio for each field of view (approx. $500 \mu \mathrm{m}$ ) and error bars correspond to SEM. (D) Live cell imaging of Pink1 and beta actin RNA in hippocampal neuron cell bodies and dendrites and as well as a mitochondrial marker (mRaspberry targeted to the mitochondrial matrix, mitoRasp). (E) Representative images of Pink1, Cox4i and Atp5f1b transcripts visualized with the MS2/PP7-split-Venus method and mitochondria detected by mitochondrially targeted Raspberry (mitoRasp). (F) Mander's correlation coefficient between the indicated transcripts and mitochondria. Student's t-test, $n=29-31$ cell bodies, $\left.p<0.01{ }^{* *}\right), p<0.0001$ 
$\left(^{* * *}\right)$. (G) Representative images for kinase-dead Pink1 transcript localization in neuronal cell bodies relative to late endosomes labelled with mCherry $(\mathrm{mCh})-\mathrm{Rab7a}$ and mitochondria labelled with mitoBFP. Scale bar $=10 \mu \mathrm{m}$. $(\mathrm{H})$ Quantification with Mander's correlation coefficients of the extent of transcript colocalization in $(G)$ with mitochondria (Mito.) and with Late Endosomes (LE). Student's t-test, $n=20$ cell bodies; $\left.\left.p<0.01{ }^{* *}\right), p<0.0001{ }^{(* * *}\right)$. Scale bars $=10 \mu \mathrm{m}(\operatorname{except}(\mathrm{A}))$.

Figure S3 corresponding to Figure 3. Pink1 mRNA is transported with mitochondria in axons and dendrites. (A) Repetition of Figure 3B alongside a schematic that represents a likely interpretation of selected elements in the kymograph. Co-transport of mRNA and mitochondria is present (yellow arrowhead). At the grey arrowhead, a Pink1 mRNA particle appears to dissociate from a moving mitochondrion and, after a period, to reassociate with a different moving mitochondrion. Another potential dissociation and brief episode of short-range mitochondrially independent movement of a Pink1 mRNA particle is marked with a blue arrow head. Scale bar $=10 \mu \mathrm{m}$. (B) Kymograph and schematic of Pink1 mRNA and mitochondrial co-transport (yellow arrowhead) in a proximal axon. Scale bar $=10 \mu \mathrm{m}$

Figure S4 corresponding to Figure 4. Representative images of Pink1-BFP. (A) Representative images for Pink1 5'UTR-BFP, BFP-Pink1 3'UTR and BFP-Pink1 C+3'UTR transcript localization. (B) Representative localization of full length Pink1 (kinase dead) transcript in the presence or absence of puromycin. Scale bars $=10 \mu \mathrm{m}$.

Figure S5 corresponding to Figure 5. Knock down of SYNJ2BP does not alter beta actin mRNA localization nor PINK1 translation or RNA stability. (A) Representative Western Blot and quantification of SYNJ2BP protein levels in neurons treated with control or SYNJ2BP shRNA for three days. Band intensity was normalized to Rpl10a and control shRNA. Data is shown as mean \pm SEM; Student's t-test; $n=3$ experiments, $p<0.05\left(^{*}\right)$. (B) Representative images of neuronal cell bodies expressing the split-Venus reporter for the Pink1 transcript and RFP-tagged DDX6. (C) Colocalization as analyzed with Mander's correlation coefficient between the beta actin transcript and P-bodies marked with RFP-DDX in control and SYNJ2BP shRNA treated neuron cell bodies. Student's t-test, $n=16-17$ cell bodies. (D) Representative images from control or SYNJ2BP shRNA treated neuron cell bodies illustrating the localization of beta actin mRNA with respect to DDX6. (E) Pink1 transcript abundance was analyzed by qPCR in control of SYNJ2BP shRNA treated neurons. Data is presented as mean \pm SEM; Student's t-test, $n=3$ biological repeats per condition $(F)$ Representative micrographs of PINK1N-BFP (compare Figure 4) expression in control and SYNJ2BP knock down neurons. Scale bar $=10 \mu \mathrm{m}$. 
Figure S6 corresponding to Figure 6. Knock down of SYNJ2 affects Pink1 mitochondrial localization in neurons. Quantification (A) and representative images (B) of mitochondrial localization of the Pink1 transcript visualized by the split-Venus method and mitoRasp in control and SYNJ2 shRNA treated hippocampal cell bodies. Expression of an shRNA-resistant SYNJ2 restored localization of Pink1 transcripts to mitochondria. Student's t-test, $n=14-20$ cell bodies, $p<0.05\left(^{*}\right)$ and $p<0.0001\left(^{* * *}\right)$. Scale bar $=10 \mu \mathrm{m}$.

\section{Figure S7 corresponding to Figure 7. Expression of SYNJ2mito does not alter beta actin} transcript localization. (A) SYNJ2-mito VQL/AAA is similarily expressed as its WT counterpart as analyzed by immunocytochemistry (ICC) against its myc-tag normalized to the co-transfected mitoRaspberry. Student's t-test, $n=20-21$ neurons. (B) Colocalization as quantified with Mander's correlation coefficient between mitochondria and beta actin transcripts. (C) Representative images from control or SYNJ2BP shRNA treated neuron cell bodies with and without expression of SYNJ2mito. Student's t-test, $n=11-18$ cell bodies; Scale bar $=10 \mu \mathrm{m}$.

Supplemental movie 1 corresponding to Figure 3. Pink1 mRNA is transported with mitochondria in dendrites.

Supplemental movie 2 corresponding to Figure 3. This movie illustrates possible association and dissociation events of Pink1 mRNA and mitochondria in a dendrite. The movie is played twice. In the first part a white arrow indicates a stationary Pink1 mRNA particle (arrow) that associates with a moving Pink1 mRNA on a moving mitochondrion (arrowheads). The second part illustrates a moving Pink1 mRNA particle that seems to split and while one part keeps moving on (arrowhead) the other stays behind (arrow). There is no detectable mitochondrial signal at the position of the stationary Pink1 mRNA particle.

Supplemental movie 3 corresponding to Figure 3. Pink1 mRNA is transported with mitochondria in axons. This movie demonstrates a detail of the kymograph presented in Fig. S2. At 18' a mitochondrion-associated mRNA particle (marked with arrowheads) enters from the right, then pauses. At 44', a second mitochondrion and its associated mRNA, which had been stable (arrowheads), begins to move.

\section{References}

Ando, M., Fiesel, F.C., Hudec, R., Caulfield, T.R., Ogaki, K., Górka-Skoczylas, P., Koziorowski, D., Friedman, A., Chen, L., Dawson, V.L., et al. (2017). The PINK1 p.I368N mutation affects protein stability and ubiquitin kinase activity. Mol. Neurodegener. 12, 32. Andreassi, C., Zimmermann, C., Mitter, R., Fusco, S., De Vita, S., Devita, S., Saiardi, A., and 
Riccio, A. (2010). An NGF-responsive element targets myo-inositol monophosphatase-1 mRNA to sympathetic neuron axons. Nat. Neurosci. 13, 291-301.

Ashrafi, G., Schlehe, J.S., LaVoie, M.J., and Schwarz, T.L. (2014). Mitophagy of damaged mitochondria occurs locally in distal neuronal axons and requires PINK1 and Parkin. J. Cell Biol. 206, 655-670.

Ben-Yaakov, K., Dagan, S.Y., Segal-Ruder, Y., Shalem, O., Vuppalanchi, D., Willis, D.E., Yudin, D., Rishal, I., Rother, F., Bader, M., et al. (2012). Axonal transcription factors signal retrogradely in lesioned peripheral nerve. EMBO J. 31, 1350-1363.

Biever, A., Glock, C., Tushev, G., Ciirdaeva, E., Dalmay, T., Langer, J.D., and Schuman, E.M. (2020). Monosomes actively translate synaptic mRNAs in neuronal processes. Science 367.

Bolam, J.P., and Pissadaki, E.K. (2012). Living on the edge with too many mouths to feed: why dopamine neurons die. Mov. Disord. 27, 1478-1483.

Briley, W.E., Bondy, M.H., Randeria, P.S., Dupper, T.J., and Mirkin, C.A. (2015).

Quantification and real-time tracking of RNA in live cells using Sticky-flares. Proc. Natl. Acad. Sci. U. S. A. 112, 9591-9595.

Castello, A., Fischer, B., Eichelbaum, K., Horos, R., Beckmann, B.M., Strein, C., Davey, N.E., Humphreys, D.T., Preiss, T., Steinmetz, L.M., et al. (2012). Insights into RNA biology from an atlas of mammalian mRNA-binding proteins. Cell 149, 1393-1406.

Chen, Y., and Dorn, G.W. (2013). PINK1-phosphorylated mitofusin 2 is a Parkin receptor for culling damaged mitochondria. Science 340, 471-475.

Cheng, H.-C., Ulane, C.M., and Burke, R.E. (2010). Clinical progression in Parkinson disease and the neurobiology of axons. Ann. Neurol. 67, 715-725.

Cioni, J.-M., Lin, J.Q., Holtermann, A. V, Koppers, M., Jakobs, M.A.H., Azizi, A., TurnerBridger, B., Shigeoka, T., Franze, K., Harris, W.A., et al. (2019). Late Endosomes Act as mRNA Translation Platforms and Sustain Mitochondria in Axons. Cell 176, 56-72.e15.

Cohen, B., Golani-Armon, A., Altman, T., Savulescu, A. F., Mhlanga, M. M., Perlson, E. and Arava, Y.S. (2021). Mitochondria serve as axonal shuttle for Cox7c mRNA through mechanism that involves its mitochondrial targeting signal. submittted to BioRxiv.

Cornelissen, T., Vilain, S., Vints, K., Gounko, N., Verstreken, P., and Vandenberghe, W. (2018). Deficiency of parkin and PINK1 impairs age-dependent mitophagy in Drosophila. Elife 7.

Cosker, K.E., Fenstermacher, S.J., Pazyra-Murphy, M.F., Elliott, H.L., and Segal, R.A. (2016). The RNA-binding protein SFPQ orchestrates an RNA regulon to promote axon viability. Nat. Neurosci. 19, 690-696.

Donnelly, C.J., Fainzilber, M., and Twiss, J.L. (2010). Subcellular Communication Through RNA Transport and Localized Protein Synthesis. Traffic 11, 1498-1505. 
Durcan, T.M., and Fon, E.A. (2015). The three 'P's of mitophagy: PARKIN, PINK1, and posttranslational modifications. Genes Dev. 29, 989-999.

Evans, C.S., and Holzbaur, E.L.F. (2020). Lysosomal degradation of depolarized mitochondria is rate-limiting in OPTN-dependent neuronal mitophagy. Autophagy 16, 962964.

Exner, N., Lutz, A.K., Haass, C., and Winklhofer, K.F. (2012). Mitochondrial dysfunction in Parkinson's disease: molecular mechanisms and pathophysiological consequences. EMBO J. 31, 3038-3062.

Fazal, F.M., Han, S., Parker, K.R., Kaewsapsak, P., Xu, J., Boettiger, A.N., Chang, H.Y., and Ting, A.Y. (2019). Atlas of Subcellular RNA Localization Revealed by APEX-Seq. Cell 178, 473-490.e26.

Garcia, M., Delaveau, T., Goussard, S., and Jacq, C. (2010). Mitochondrial presequence and open reading frame mediate asymmetric localization of messenger RNA. EMBO Rep. 11, 285-291.

Greenberg, J.R. (1979). Ultraviolet light-induced crosslinking of mRNA to proteins. Nucleic Acids Res. 6, 715-732.

Gumy, L.F., Yeo, G.S.H., Tung, Y.-C.L., Zivraj, K.H., Willis, D., Coppola, G., Lam, B.Y.H., Twiss, J.L., Holt, C.E., and Fawcett, J.W. (2011). Transcriptome analysis of embryonic and adult sensory axons reveals changes in mRNA repertoire localization. RNA 17, 85-98.

Hanz, S., Perlson, E., Willis, D., Zheng, J.-Q., Massarwa, R., Huerta, J.J., Koltzenburg, M., Kohler, M., van-Minnen, J., Twiss, J.L., et al. (2003). Axoplasmic importins enable retrograde injury signaling in lesioned nerve. Neuron 40, 1095-1104.

Harbauer, A.B. (2017). Mitochondrial health maintenance in axons. Biochem. Soc. Trans. 45, 1045-1052.

Harbauer, A.B., Zahedi, R.P., Sickmann, A., Pfanner, N., and Meisinger, C. (2014). The protein import machinery of mitochondria - A regulatory hub in metabolism, stress, and disease. Cell Metab. 19, 357-372.

Hegde, R.S., and Bernstein, H.D. (2006). The surprising complexity of signal sequences. Trends Biochem. Sci. 31, 563-571.

Hengst, U., Deglincerti, A., Kim, H.J., Jeon, N.L., and Jaffrey, S.R. (2009). Axonal elongation triggered by stimulus-induced local translation of a polarity complex protein. Nat. Cell Biol. 11, 1024-1030.

Hillefors, M., Gioio, A.E., Mameza, M.G., and Kaplan, B.B. (2007). Axon viability and mitochondrial function are dependent on local protein synthesis in sympathetic neurons. Cell. Mol. Neurobiol. 27, 701-716.

Holt, C.E., and Schuman, E.M. (2013). The central dogma decentralized: new perspectives on RNA function and local translation in neurons. Neuron 80, 648-657. 
Hsieh, C.-H., Shaltouki, A., Gonzalez, A.E., Bettencourt da Cruz, A., Burbulla, L.F., St Lawrence, E., Schüle, B., Krainc, D., Palmer, T.D., and Wang, X. (2016). Functional Impairment in Miro Degradation and Mitophagy Is a Shared Feature in Familial and Sporadic Parkinson's Disease. Cell Stem Cell 19, 709-724.

Hsu, F., and Mao, Y. (2015). The structure of phosphoinositide phosphatases: Insights into substrate specificity and catalysis. Biochim. Biophys. Acta 1851, 698-710.

Hung, V., Lam, S.S., Udeshi, N.D., Svinkina, T., Guzman, G., Mootha, V.K., Carr, S.A., and Ting, A.Y. (2017). Proteomic mapping of cytosol-facing outer mitochondrial and ER membranes in living human cells by proximity biotinylation. Elife 6 .

Kar, A.N., Vargas, J.N.S., Chen, C.-Y., Kowalak, J.A., Gioio, A.E., and Kaplan, B.B. (2017). Molecular determinants of cytochrome $\mathrm{C}$ oxidase IV mRNA axonal trafficking. Mol. Cell. Neurosci. 80, 32-43.

Katayama, H., Kogure, T., Mizushima, N., Yoshimori, T., and Miyawaki, A. (2011). A sensitive and quantitative technique for detecting autophagic events based on lysosomal delivery. Chem. Biol. 18, 1042-1052.

Kitada, T., Pisani, A., Porter, D.R., Yamaguchi, H., Tscherter, A., Martella, G., Bonsi, P., Zhang, C., Pothos, E.N., and Shen, J. (2007). Impaired dopamine release and synaptic plasticity in the striatum of PINK1-deficient mice. Proc. Natl. Acad. Sci. 104, 11441-11446. Kuzniewska, B., Cysewski, D., Wasilewski, M., Sakowska, P., Milek, J., Kulinski, T.M., Winiarski, M., Kozielewicz, P., Knapska, E., Dadlez, M., et al. (2020). Mitochondrial protein biogenesis in the synapse is supported by local translation. EMBO Rep. 21, 789164.

Lazarou, M., Jin, S.M., Kane, L. a, and Youle, R.J. (2012). Role of PINK1 binding to the TOM complex and alternate intracellular membranes in recruitment and activation of the E3 ligase Parkin. Dev. Cell 22, 320-333.

Lee, J.J., Sanchez-Martinez, A., Zarate, A.M., Benincá, C., Mayor, U., Clague, M.J., and Whitworth, A.J. (2018). Basal mitophagy is widespread inDrosophilabut minimally affected by loss of Pink1 or parkin. J. Cell Biol. jcb.201801044.

Lin, W., and Kang, U.J. (2008). Characterization of PINK1 processing, stability, and subcellular localization. J. Neurochem. 106, 464-474.

Lin, M.-Y., Cheng, X.-T., Tammineni, P., Xie, Y., Zhou, B., Cai, Q., and Sheng, Z.-H. (2017). Releasing Syntaphilin Removes Stressed Mitochondria from Axons Independent of Mitophagy under Pathophysiological Conditions. Neuron 94, 595-610.e6. Lionnet, T., Czaplinski, K., Darzacq, X., Shav-Tal, Y., Wells, A.L., Chao, J.A., Park, H.Y., de Turris, V., Lopez-Jones, M., and Singer, R.H. (2011). A transgenic mouse for in vivo detection of endogenous labeled mRNA. Nat. Methods 8, 165-170.

Margeot, A., Blugeon, C., Sylvestre, J., Vialette, S., Jacq, C., and Corral-Debrinski, M. (2002). In Saccharomyces cerevisiae, ATP2 mRNA sorting to the vicinity of mitochondria is 
essential for respiratory function. EMBO J. 21, 6893-6904.

Maris, C., Dominguez, C., and Allain, F.H.-T. (2005). The RNA recognition motif, a plastic RNA-binding platform to regulate post-transcriptional gene expression. FEBS J. 272, 21182131.

McWilliams, T.G., Prescott, A.R., Montava-Garriga, L., Ball, G., Singh, F., Barini, E., Muqit, M.M.K., Brooks, S.P., and Ganley, I.G. (2018). Basal Mitophagy Occurs Independently of PINK1 in Mouse Tissues of High Metabolic Demand. Cell Metab. 0.

Miller, K.E., and Sheetz, M.P. (2004). Axonal mitochondrial transport and potential are correlated. J. Cell Sci. 117, 2791-2804.

Misgeld, T., and Schwarz, T.L. (2017). Mitostasis in Neurons: Maintaining Mitochondria in an Extended Cellular Architecture. Neuron 96, 651-666.

Narendra, D.P., Jin, S.M., Tanaka, A., Suen, D.-F., Gautier, C. a, Shen, J., Cookson, M.R., and Youle, R.J. (2010). PINK1 is selectively stabilized on impaired mitochondria to activate Parkin. PLoS Biol. 8, e1000298.

Nemoto, Y., and De Camilli, P. (1999). Recruitment of an alternatively spliced form of synaptojanin 2 to mitochondria by the interaction with the PDZ domain of a mitochondrial outer membrane protein. EMBO J. 18, 2991-3006.

Nemoto, Y., Arribas, M., Haffner, C., and DeCamilli, P. (1997). Synaptojanin 2, a novel synaptojanin isoform with a distinct targeting domain and expression pattern. J. Biol. Chem. 272, 30817-30821.

Okatsu, K., Kimura, M., Oka, T., Tanaka, K., and Matsuda, N. (2015). Unconventional PINK1 localization mechanism to the outer membrane of depolarized mitochondria drives Parkin recruitment. J. Cell Sci. jcs.161000-.

Ostroff, L.E., Santini, E., Sears, R., Deane, Z., Kanadia, R.N., LeDoux, J.E., Lhakhang, T., Tsirigos, A., Heguy, A., and Klann, E. (2019). Axon TRAP reveals learning-associated alterations in cortical axonal mRNAs in the lateral amgydala. Elife 8.

Overly, C.C., Rieff, H.I., and Hollenbeck, P.J. (1996). Organelle motility and metabolism in axons vs dendrites of cultured hippocampal neurons. J. Cell Sci. 109 ( Pt 5, 971-980.

Pickrell, A.M., and Youle, R.J. (2015). The Roles of PINK1, Parkin, and Mitochondrial Fidelity in Parkinson's Disease. Neuron 85, 257-273.

Qin, W., Myers, S.A., Carey, D.K., Carr, S.A., and Ting, A.Y. (2020). Functional proximity mapping of RNA binding proteins uncovers a mitochondrial mRNA anchor that promotes stress recovery. BioRxiv.

Raab-Graham, K.F., Haddick, P.C.G., Jan, Y.N., and Jan, L.Y. (2011). Activity- and mTORDependent Suppression of Kv1.1 Channel mRNA Translation in Dendrites. Science (80-. ). 144, 1-6.

Schwanhäusser, B., Busse, D., Li, N., Dittmar, G., Schuchhardt, J., Wolf, J., Chen, W., and 
Selbach, M. (2011). Global quantification of mammalian gene expression control. Nature 473, 337-342.

Sfakianos, A.P., Whitmarsh, A.J., and Ashe, M.P. (2016). Ribonucleoprotein bodies are phased in. Biochem. Soc. Trans. 44, 1411-1416.

Shigeoka, T., Jung, H., Jung, J., Turner-Bridger, B., Ohk, J., Lin, J.Q., Amieux, P.S., Holt, C.E., Aakalu, G., Smith, W.B., et al. (2016). Dynamic Axonal Translation in Developing and Mature Visual Circuits. Cell 0, 489-502.

Sliter, D.A., Martinez, J., Hao, L., Chen, X., Sun, N., Fischer, T.D., Burman, J.L., Li, Y., Zhang, Z., Narendra, D.P., et al. (2018). Parkin and PINK1 mitigate STING-induced inflammation. Nature 561, 258-262.

Sotelo-Silveira, J., Crispino, M., Puppo, A., Sotelo, J.R., and Koenig, E. (2008). Myelinated axons contain beta-actin mRNA and ZBP-1 in periaxoplasmic ribosomal plaques and depend on cyclic AMP and F-actin integrity for in vitro translation. J. Neurochem. 104, 545-557.

Spillane, M., Ketschek, A., Merianda, T.T., Twiss, J.L., and Gallo, G. (2013). Mitochondria coordinate sites of axon branching through localized intra-axonal protein synthesis. Cell Rep. $5,1564-1575$.

Terenzio, M., Koley, S., Samra, N., Rishal, I., Zhao, Q., Sahoo, P.K., Urisman, A., Marvaldi, L., Oses-prieto, J.A., Forester, C., et al. (2018). Locally translated mTOR controls axonal local translation in nerve injury. Science (80-. ). 1359, 1416-1421.

Tsai, P.I., Lin, C.H., Hsieh, C.H., Papakyrikos, A.M., Kim, M.J., Napolioni, V., Schoor, C., Couthouis, J., Wu, R.M., Wszolek, Z.K., et al. (2018). PINK1 Phosphorylates MIC60/Mitofilin to Control Structural Plasticity of Mitochondrial Crista Junctions. Mol. Cell 69, 744-756.e6. Vardi-Oknin, D., and Arava, Y. (2019). Characterization of factors involved in localized translation near mitochondria by ribosome-proximity labeling. Front. Cell Dev. Biol. 7, 305. Verma, P., Chierzi, S., Codd, A.M., Campbell, D.S., Meyer, R.L., Holt, C.E., and Fawcett, J.W. (2005). Axonal protein synthesis and degradation are necessary for efficient growth cone regeneration. J. Neurosci. 25, 331-342.

Verner, K. (1993). Co-translational protein import into mitochondria : an alternativeview. Trends Biochem. Sci.

Vincow, E.S., Merrihew, G., Thomas, R.E., Shulman, N.J., Beyer, R.P., MacCoss, M.J., and Pallanck, L.J. (2013). The PINK1-Parkin pathway promotes both mitophagy and selective respiratory chain turnover in vivo. Proc. Natl. Acad. Sci. U. S. A. 110, 6400-6405.

Vives-Bauza, C., Zhou, C., Huang, Y., Cui, M., de Vries, R.L. a, Kim, J., May, J., Tocilescu, M.A., Liu, W., Ko, H.S., et al. (2010). PINK1-dependent recruitment of Parkin to mitochondria in mitophagy. Proc. Natl. Acad. Sci. U. S. A. 107, 378-383.

Wang, X., Winter, D., Ashrafi, G., Schlehe, J., Wong, Y.L., Selkoe, D., Rice, S., Steen, J., LaVoie, M.J., and Schwarz, T.L. (2011). PINK1 and Parkin target Miro for phosphorylation 
and degradation to arrest mitochondrial motility. Cell 147, 893-906.

Wilbertz, J.H., Voigt, F., Horvathova, I., Roth, G., Zhan, Y., and Chao, J.A. (2019). SingleMolecule Imaging of mRNA Localization and Regulation during the Integrated Stress Response. Mol. Cell 73, 946-958.e7.

Wu, B., Chen, J., and Singer, R.H. (2014). Background free imaging of single mRNAs in live cells using split fluorescent proteins. Sci. Rep. 4, 3615.

Yamano, K., Matsuda, N., and Tanaka, K. (2016). The ubiquitin signal and autophagy: an orchestrated dance leading to mitochondrial degradation. EMBO Rep. 17, 300-316.

Yoon, B.C., Jung, H., Dwivedy, A., O’Hare, C.M., Zivraj, K.H., and Holt, C.E. (2012). Local translation of extranuclear lamin B promotes axon maintenance. Cell 148, 752-764.

Yousefi, R., Fornasiero, E.F., Cyganek, L., Montoya, J., Jakobs, S., Rizzoli, S.O., Rehling, P., and Pacheu-Grau, D. (2021). Monitoring mitochondrial translation in living cells. EMBO Rep. 22, 1-12.

Zivraj, K.H., Tung, Y.C.L., Piper, M., Gumy, L., Fawcett, J.W., Yeo, G.S.H., and Holt, C.E. (2010). Subcellular profiling reveals distinct and developmentally regulated repertoire of growth cone mRNAs. J. Neurosci. 30, 15464-15478.

\section{Supplemental information}

\section{Key Resources Table}

\section{Antibodies}

REAGENT or RESOURCE

Rabbit polyclonal PINK1 antibody

Novus Biologicals

GAPDH antibody

Mouse monoclonal ATP5A antibody

[15H4C4]

SOURCE

IDENTIFIER

Rabbit polyclonal ATP5B antibody

Abcam

Cat\# BC100-494

RRID: AB_10127658

Mouse monoclonal beta actin antibody (AC-74)

Sigma-Aldrich

Sigma-Aldrich

Mouse monoclonal RPL10A antibody [3G2]

Abcam

Cat\# ab14748

RRID:AB_301447

Rabbit polyclonal SYNJ2BP antibody Proteintech

Cat\# HPA001520

RRID: AB_1078243

Cat\# A2228

RRID:AB 476697

Cat\# ab55544

RRID:AB_945301

Cat\# 15666-1-AP, RRID:AB_2201149

Mouse polyclonal SYNJ2BP antibody Sigma-Aldrich Cat\# SAB1400613-

50UG, RRID:

$A B \_1857695$

Rabbit polyclonal Synj2 antibody

Proteintech

Cat\# 13893-1-AP, RRID: AB 2255784 
Rabbit RHOT1 monoclonal antibody $(4 \mathrm{H} 4)$

Sigma-Aldrich

Chicken polyclonal MAP2 antibody

Novus Biologicals

Mouse monoclonal betalll-tubulin antibody (2G10)

Thermo Fisher

Mouse monoclonal c-Myc antibody (9E10) Novus Biologicals

Rabbit monoclonal Myc-tag antibody (71D10)

Rabbit monoclonal HA-tag antibody (C29F4)

Goat anti-Rabbit IgG IRDye $800 \mathrm{CW}$ Conjugated antibody

Goat anti-Mouse IgG, IRDye 680RD Conjugated antibody

Peroxidase AffiniPure Goat anti-Rabbit antibody

Horseradish Peroxidase Conjugated Goat anti-Mouse lgG, Fc fragment Polyclonal antibody

Alexa488-conjugated goat anti-mouse antibody

Alexa488-conjugated goat anti-rabbit antibody

Alexa647-conjugated goat-anti chicken antibody

Abberior STAR 635P, goat anti-mouse antibody

\section{Bacterial and Virus Strains}

DH5alpha

Chemicals, Peptides, and Recombinant

\section{Proteins}

Poly-L-Lysine

Laminin

Antimycin A

Cycloheximide

Puromycin

Fluoromount $\mathrm{G}$

\section{Critical Commercial Assays}

Microfluidic Neuron Device

RNeasy Mini Kit

qScript $^{\mathrm{TM}}$ cDNA SuperMix

PerfeCTa SYBR $®$ Green FastMix

TRIZOL

Thermo Fisher
Cell Signaling

Technology

Cell Signaling

Technology

LI-COR Biosciences

LI-COR Biosciences

Jackson

ImmunoResearch Labs

Sigma

Thermo Fisher

Thermo Fisher

Abberior

Invitrogen

Sigma

Life Technologies

Sigma

Sigma

RPI

Enzo Life Sciences

Southern Biotech

Invitrogen

XONA Microfluidics

QIAGEN

Quantabio

Quantabio

Thermo Fisher
Cat\# WH0055288M1-

100UG, RRID:

AB 1843347

Cat\# NB300-213,

RRID: AB_2138178

Cat\# MA1-118, RRID:

AB_2536829

Cat\# NB600-302,

RRID:AB_2037060

Cat\# 2278,

RRID:AB_490778

Cat\# 3724, RRID:

AB_1549585

Cat\# 925-32211,

RRID:AB_2651127

Cat\# 925-68070,

RRID:AB 2651128

Cat\# 111-035-144,

RRID:AB 2307391

Cat\# A0168,

RRID:AB_257867

Cat\# A-11001, RRID:AB_2534069

Cat\# A-11008, RRID:

AB_143165

Cat\# A-21449, RRID:

AB_2535866

Cat\# ST635P-1001-

500UG

Cat\# 18265-017

Cat\# P2636-25MG

Cat\# 23017-015

Cat\# A8674-25MG

Cat\# C4859

Cat\# P33020-0.025

Cat\# BML-GR312-

0050

Cat\# 0100-01

Cat\# 00-4958-02

Cat\# RD450

Cat\# 204141

Cat\# 95048-025

Cat\# 95072-250

Cat\# 15596-026 
RNAscope 2.5 HD Assay - RED

RNAscope Multiplex Assay

Opal 570

Probes for mouse Pink1

Probes for mouse beta actin

RNAscope Protease III

Duolink ${ }^{\mathrm{TM}}$ Proximity ligation assay

Experimental Models:

Organisms/Strains

Long-Evans rats

cos-7

HEK293T/17

C57BL6 mice

BR33 iPSC line

\section{Oligonucleotides}

Primer for rat PINK1 qRT-PCR forward: gcgaagccatcttaagcaaa

Primer for rat PINK1 qRT-PCR reverse: TGCTACCCCCACACTACTAC

Primer for gamma actin qRT-PCR forward:

CAGATGGACTGAGCAGGTGCCAG

Primer for rat gamma actin qRT-PCR reverse: CAGCAACAAGTTCTACGATCC Primer for 12S Mitochondrial rRNA qRTPCR forward: CCCAGAGAACATTAGG

Primer for 12S Mitochondrial rRNA qRT-

$P C R$ reverse:

Advanced Cell

Diagnostics

Advanced Cell

Diagnostics

Akoya Biosciences

Advanced Cell

Diagnostics

Advanced Cell

Diagnostics

Advanced Cell

Diagnostics

Sigma

Charles River

ATCC

ATCC

Jackson Laboratory

Rush Alzheimer

Disease Center

this study

$\mathrm{n} / \mathrm{a}$

this study

$\mathrm{n} / \mathrm{a}$

Willis et al, 2005

$\mathrm{n} / \mathrm{a}$

this study

$\mathrm{n} / \mathrm{a}$

Willis et al, 2005

$\mathrm{n} / \mathrm{a}$

Willis et al, 2005

$\mathrm{n} / \mathrm{a}$

GAGGAGGGTGACGGGCGG

Primer for rat beta actin qRT-PCR forward:

ACACTGTGCCCATCTATG

this study

$\mathrm{n} / \mathrm{a}$

Primer for beta actin $\mathrm{QRT}$-PCR reverse:

GCTGTGGTGGTGAAGCTGTAG

this study

$\mathrm{n} / \mathrm{a}$

Primer for rat PINK1-MBSPBS qRT-PCR

forward:

this study

$\mathrm{n} / \mathrm{a}$

aacctggagtgtgaggcact

Primer for rat PINK1-MBSPBS qRT-PCR

reverse:

this study

$\mathrm{n} / \mathrm{a}$

Cat\# 322360

Cat\# 323100

Cat\# FP1488001KT

Cat\# 524081

Cat\# 316741-C3

Cat\# 322340

Cat\# DUO92101

RRID:RGD_2308852

Cat\# CRL-1651,

RRID:CVCL_0224

Cat\# CRL-11268G-1, RRID:CVCL_UE07

RRID:IMSR_JAX:0006

64

$\mathrm{n} / \mathrm{a}$

tcgccgaagggcgaattct 
Primer for rodent SYNJ2a qRT-PCR forward: GCTCACTGCTGCAAAGACAT Primer for rodent SYNJ2a qRT-PCR reverse:

AAGCATCTACGGGTGTCAGT

Primer for Rrbp1 qRT-PCR forward: GGCATCTTCCTGGTGTC

Primer for Rrbp1 qRT-PCR reverse : GAGTTTTCGCCATCTCCTTG

\section{Recombinant DNA}

DsRed-Mito plasmid YFP-Parkin

AAV-GFP

AAV-PINK1-12xMBS-PBS

mito-Raspberry-7

AnkyrinG-mCherry

mito-BFP

mCherry-Rab7a

Pcr4-12xMBS-PBS

Rat PINK1

PINK1-12xMBS-PBS

Beta actin-12xMBS-PBS

COX4i-12xMBS-PBS

ATP5F1B-12xMBS-PBS

PINK1-KD-12xMBS-PBS

PINK1-N-BFP-12xMBS-PBS

PINK1-C-BFP-12xMBS-PBS

PINK1-5‘utr-BFP-12xMBS-PBS

PINK1-3'utr-BFP-12xMBS-PBS

PINK1-5'utr+MTS-BFP-12xMBS-PBS

PINK1-N-atg-BFP-12xMBS-PBS

ubc-nls-ha-MCP-VenusN-IRES-nls-ha$\mathrm{PCP}-$ VenusC

ubc-ha-MCP-VenusN-IRES-PCP-VenusCzipcode

ubc-ha-MCP-VenusN-P2A-nls-ha-PCP-

VenusC-zipcode

Sigma Control shRNA

Sigma SYNJ2BP shRNA this study

$\mathrm{n} / \mathrm{a}$

this study

$\mathrm{n} / \mathrm{a}$

this study

$\mathrm{n} / \mathrm{a}$

this study

$\mathrm{n} / \mathrm{a}$

Clontech

Cat\# 632421

Narendra et al., 2008

RRID:Addgene_23955

Park et al, 2008

$\mathrm{n} / \mathrm{a}$

this study

Gift from Michael

Davidson

$\mathrm{n} / \mathrm{a}$

Leterrier et al, 2011

RRID:Addgene_55931

Friedman et al, 2011

RRID:Addgene_42566

RRID:Addgene_49151

gift from Michael

Davidson

RRID:Addgene_55127

Wu et al, 2014

RRID:Addgene_52984

Transomic

Cat\# BC169047

Recloned from Pcr4-

12xMBS-PBS and rat

this study

PINK1 in UBC-pHAGE

backbone RRID:

Addgene_52985

this study

$\mathrm{n} / \mathrm{a}$

this study

$\mathrm{n} / \mathrm{a}$

this study

$\mathrm{n} / \mathrm{a}$

this study

$\mathrm{n} / \mathrm{a}$

this study

$\mathrm{n} / \mathrm{a}$

this study

$\mathrm{n} / \mathrm{a}$

this study

$\mathrm{n} / \mathrm{a}$

this study

$\mathrm{n} / \mathrm{a}$

this study

$\mathrm{n} / \mathrm{a}$

this study

$\mathrm{n} / \mathrm{a}$

Wu et al, 2014

RRID:Addgene_52985

this study

$\mathrm{n} / \mathrm{a}$

this study

$\mathrm{n} / \mathrm{a}$

SHC016

Sigma

Puromycin cassette was replaced by BFP

TRCN0000139049 
Sigma SYNJ2BP shRNA

Origene Control shRNA

Origene CLUH shRNA

tagRFP-T-DDX6

Myc-SYNJ2BP

Myc-SYNJ2BP shRNA resistant

FLAG-SYNJ2a

SYNJ1-FLAG

Myc-SYNJ2a-mito

Myc-SYNJ2a-mito VQL/AAA

Myc-SYNJ2aRRM-mito

Myc-SYNJ2aRRM-mito VQL/AAA

V5-GFP-RRBP1

PINK1-N-Kaede

Mito-Kaede

Mito-Keima

\section{Software and Algorithms}

ImageJ

Kymolyzer Plugin

$\mathrm{R}$

Image Studio Lite
Sigma

Origene

Origene

Wilbertz et al. 2019

this study

this study

Nemoto et al., 1997

Nemoto et al., 1997

this study

this study

this study

this study

Hung et al. 2017

this study

this study

Vargas et al. 2019

Schindelin et al., 2015

Pekkurnaz et al., 2014

The R Foundation

LI-COR
Puromycin cassette was replaced by BFP

TRCN0000050377

Puromycin cassette was replaced by BFP

TR30021

TL517906 / FI501310

RRID:Addgene_11994 7

$\mathrm{n} / \mathrm{a}$

$\mathrm{n} / \mathrm{a}$

$\mathrm{n} / \mathrm{a}$

$\mathrm{n} / \mathrm{a}$

Recloned from Myc-

SYNJ2BP and FLAGSYNJ2a

$\mathrm{n} / \mathrm{a}$

$\mathrm{n} / \mathrm{a}$

$\mathrm{n} / \mathrm{a}$

RRID: Addgene_92150

PINK1 aa 1-225

inserted into $\mathrm{N} 1-$ Kaede

RRID:Addgene_54726

COX8 aa 1-36 inserted

into N1-Kaede

RRID:Addgene_54726;

kind gift from Michael

Davidson

RRID::Addgene_13162

6

RRID: SCR_001935

$\underline{n / a}$

https://www.r-

project.org/

RRID:SCR_001905

https://www.licor.com/bi o/products/software/im

age_studio_lite/

RRID:SCR_013715

\section{Contact for Reagent and Resource Sharing}

Further information and requests for resources and reagents should be directed to and will be fulfilled by the corresponding author Thomas L. Schwarz.

Thomas.schwarz@childrens.harvard.edu 


\section{Experimental Model and Subject Details}

Primary fibroblast and neuron cultures were obtained from E18 (embryonic day 18) Long/Evans rat pups or on E16.5 from mouse pups. Pregnant females from timed matings were delivered from Charles River Laboratories and housed overnight in the animal facility. Rat and mouse procedures were approved by the Institutional Animal Care Committee (IACUC) at the Boston Children's Hospital and the MPI of neurobiology. BR33 iPSCs were obtained from Rush Alzheimer Disease Center.

\section{Cell culture preparation}

Primary cell cultures were prepared as described in Shlevkov et al. (2016). Hippocampal neurons were obtained by euthanizing the pregnant female with $\mathrm{CO}_{2}$ and recovery of the $\mathrm{E} 18$ embryos from the abdomen. Hippocampi were dissected and placed in chilled dissociation medium (Ca2+-free HBSS with 100 mM MgCl2, 10 mM kynurenic acid, and $100 \mathrm{mM}$ Hepes), and enzymatically dissociated with Papain/l-cysteine (Worthington Biochemical Corporation). After addition of Trypsin inhibitor (Sigma-Aldrich), tissue was triturated 10-15 times with a P1000 pipet until clumps disappeared. Neurons were resuspended in Neurobasal medium supplemented with B27 (Gibco/Life Technologies), L-glutamine, and penicillin/streptomycin (NB+PSG+B27) and plated on $20 \mu \mathrm{g} / \mathrm{mL}$ poly-L-Lysine (Sigma- Aldrich) and $3.5 \mu \mathrm{g} / \mathrm{mL}$ laminin (ThermoFisher Scientific) coated glass bottom plates (CellVis) or acid washed glass coverslips (1.5mm, Warner Instruments). $50 \%$ of the medium was replaced every $2-3$ days with fresh $\mathrm{NB}+\mathrm{PSG}+\mathrm{B} 27$. Transfections were performed at day in vitro (DIV) 5-7 and imaging at DIV7DIV9. Fibroblasts were obtained from E18 rat embryos by standard methods and cultured in DMEM+20\% FBS. Cells were maintained in T75 flasks or frozen in 10\% DMSO for future use.

iPSCs were plated on Matrigel-coated plates (Corning, 354234) and cultured growth factor reduced $\mathrm{mTeSR}$ media (StemCell Technologies, 05857) supplemented with ROCK inhibitor (10 uM; StemCell Technologies \#72304) at a density of $100 \mathrm{~K}$ cells $/ \mathrm{cm}^{2}$. iPSCs were then transduced with lentivirus packaged pTet-O-NGN2-puro and Fudelta GW-rtTA plasmids. NGN2-transduced iPSCs were thawed in StemFlex media with ROCK inhibitor. Once cells were reached $75-80 \%$ confluency (day 1 ), cells were exposed to KnockOut media (Gibco 10829.018) supplemented with KnockOut Serum Replacement (Invitrogen 10928-028), 1\% MEM non-essential amino acids (Invitrogen 11140), 1\% GlutaMAX (Gibco 35050061) and 0.1\% BME (Invitrogen 21985-023) (KSR) containing doxycycline $(2 \mu \mathrm{g} / \mathrm{ml}$, Sigma, D9891-5g) to induce NGN2 expression. On day 2, the media was changed to equal volumes of KSR and N2B media (DMEM F12 supplemented with 1\% GlutaMAX, 3\% dextrose and N2-Supplement B; StemCell Technologies 07156) with puromycin (5 $\mu \mathrm{g} / \mathrm{ml}$; Life Technologies, A11138-03) and doxycycline to select for transduced cells. On day 3 , cells were feed with N2B media 
containing media with B27 (1:100; Life technologies, 17504-044), puromycin, and doxycycline. On day 4, the cells were dissociated with Accutase (Gibco, A11105) and frozen down in freezing media containing $1: 1$ ratio of $20 \%$ DMSO and Neurobasal media (NBM, Gibco 21103049) supplemented with B27, 10ng/mL BDNF (Peprotech, 450-02), 10ng/mL CNTF (Peprotech, 450-13), and 10ng/mL GDNF (Peprotech, 450-10), ROCK inhibitor, puromycin, and doxycycline. These NGN2-induced neurons were plated on Matrigel coated plates and grown in NBM media containing B27, BDNF, CTNF, GDNF, puromycin and doxycycline. All treatments were carried out on DIV14.

\section{Method Details}

\section{Constructs}

DsRed-Mito plasmid (Clontech) and shRNA plasmids targeting CLUH (Origene) and SYNJ2BP (pLKO1.1, Sigma), as well as control plasmids were purchased from the respective vendors. The puromycin cassette of pLKO1.1 was replaced with BFP amplified from mito-BFP (Addgene 49151) using BamHI and Nsil restriction enzymes. YFP-Parkin, mito-Raspberry-7, mito-BFP, tagRFP-T-DDX6, AnkyrinG-mCherry, mCherry-Rab7a and mito-Keima were acquired from Addgene (23955, 55931, 49151, 119947, 4256655127 and 131626 respectively). Plasmids encoding shRNA against SYNJ2 and SYNJ2BP were purchased in pLKO from Sigma (TRCN0000050377 and TRCN0000139049 respectively) as well as a control shRNA plasmid (TR30021). The Puromycin cassette in pLKO was replaced by BFP using restriction enzymes. PINK1 cDNA was purchased from Transomic and inserted into the UBC-pHAGE backbone using Notl and Clal restriction enzymes, while at the same time adding an EcoRI restriction site downstream of the 3'UTR. In order to insert the 12xMBS-PBS cassette derived from Addgene plasmid 52984, the EcoRI site in the UBC-pHAGE backbone was destroyed by site-directed mutagenesis and the EcoRI digestion product of Pcr4-12xMBS-PBS (Addgene 52984) was inserted into the newly introduced EcoRI site downstream of the PINK1 3'UTR. The correct orientation was verified by sequencing. Beta actin-12xMBS-PBS, Cox4i12xMBS-PBS and Atp5f1b-12xMBS-PBS were constructed by replacing the PINK1 sequence with either beta actin, Cox4i or Atp5f1b sequence amplified from rat hippocampal cDNA using Notl and BamHI restriction enzymes. PINK1 kinase dead (KD) mutation was introduced by site-directed mutagenesis. Constructs with portions of PINK1 were derived from the KD mutant by digesting the plasmid with BamHI and replacing the C-terminal part of PINK1 with BFP derived from mitoBFP. Further modifications were achieved using restriction-free cloning (van den Ent and Löwe, 2006). Modification of the split-Venus construct (Addgene plasmid 52985) was performed by Gibson Assembly (Gibson et al., 2009) and included the addition of the rat beta actin zipcode derived from beta-actin-12xMBS-PBS, replacement of the IRES with a P2A ribosomal skip site and removal of the nuclear targeting signal(s). PINK1-N-Kaede and mitoKaede were generated by insertion of PINK1 Aa 1-624 or Cox8a Aa 1-36 (amplified from mito- 
Raspberry) in frame before N1-Kaede (Addgene 54726; kind gift from Michael Davidson) using restriction enzyme digestion.

Myc-tagged SYNJ2BP was constructed using mycOmp25-phageNco-forward CTGACccatggacATGGAGCAGAAACTCATCTCTGAAGAGGATCTGAACGGACGGGTGGAT TATTTAG and Omp25-phageCla-reverse CTCTAATCGATtcaGAGCTGCTTTCGGTATC primers and inserted into the UBC-pHAGE backbone using Ncol and Clal restriction sites. A shRNA resistant version was constructed using site-directed mutagenesis to introduce five silent mutations in the shRNA targeting region. FLAG-SYNJ2a and SYNJ1-FLAG were kind gifts from Pietro De Camilli (Nemoto et al., 1997). An outer membrane targeted version of SYNJ2a was constructed using restriction-free cloning to replace the cytosolic part of mycSYNJ2BP with SYNJ2a, resulting in SYNJ2 (amino acids 1-1218) fused to the SYNJ2BP transmembrane domain (amino acids 110-145). Point mutations in the RRM domain were introduced using site-directed mutagenesis. For expression in HEK cells and for CLIP a shorter version was generated starting at amino acid 880 through restriction-free cloning. For AAVproduction, the PINK1-12xMBS-PBS sequence was also inserted in pAAV-MCS (Stratagene) using the Xhol and Hindl sites. The control plasmid AAV-GFP has been described before (Park et al, 2008). AAV-particles were produced at Boston Children's Hospital viral core.

\section{Neuronal cultures in microfluidic devices}

RD450 microfluidic neuron devices (XONA Microfluidics) were used as described before (Ashrafi et al., 2014). Briefly, the devices were sterilized by spraying with $70 \%$ Ethanol and dried in a tissue culture hood. The dry devices were attached to coverslips or 6-well glass bottom plates coated overnight with $20 \mu \mathrm{g} / \mathrm{mL}$ poly-L-Lysine and $3.5 \mu \mathrm{g} / \mathrm{mL}$ laminin that had been washed twice with distilled water and left to dry for 2-3 min under the hood. Dissociated hippocampal neurons were pelleted at $1500 \mathrm{~g}$ for $4 \mathrm{~min}$, resuspended at a final concentration of $20 \times 10^{3} / \mu \mathrm{l}$ in $\mathrm{NB}+\mathrm{PSG}+\mathrm{B} 27.5 \mu \mathrm{l}$ were plated into one of the somal compartments and incubated at $37^{\circ} \mathrm{C}$ in $5 \% \mathrm{CO} 2$ for 15 min before filling up the wells with NB+PSG+B27. 50\% of the medium was replaced every $2-3$ days with fresh NB+PSG+B27.

\section{Mitophagy detection}

\section{Parkin translocation}

Rat hippocampal neurons in microfluidic devices were transfected for four hours on DIV6 with mito-dsRed and YFP-Parkin using lipofectamine 2000 transfection reagent (Thermo Fisher) in medium lacking B27. On DIV8 cells were incubated in Hibernate E (BrainBits) with or without $70 \mu \mathrm{M}$ Cycloheximide (Sigma) for $4 \mathrm{~h}$, before live cell imaging at a spinning disk microscope (Yokogawa CSU-X1, Olympus IX81) equipped with an electron-multiplying charge-coupled device camera (Andor iXon; Oxford instruments) using a 40×/NA 1.3 oil immersion lens and 
Metamorph software (Molecular Devices). Images were taken before and 20 min after addition of $40 \mu \mathrm{M}$ Antimycin A (Sigma) in the axonal chamber, leaving all settings identical, including detector sensitivity and camera exposure time.

\section{Mito-mKeima mitophagy index}

Mouse hippocampal neurons were seeded in 24-well glass bottom plates (CellVis) at a density of $100^{*} 10^{3}$ and maintained as described above. On DIV6 Neurons were transfected for $20 \mathrm{~min}$ with mito-mKeima and shRNA against SYNJ2BP or Control shRNA using lipofectamine 2000 transfection reagent (Thermo Fisher) in medium lacking B27. On DIV9 cells were incubated in Hibernate $E$ (BrainBits) with or without $40 \mu \mathrm{M}$ Antimycin A (Sigma) for $1 \mathrm{~h}$ before live-imaging at the Imaging Facility of Max Planck Institute of Biochemistry, Martinsried, on a LEICA (Wetzlar, Germany) SP8 FALCON confocal laser scanning microscope equipped with a HCX PL APO 63x/1.2 motCORR CS water immersion objective. Keima green was excited at 442 $\mathrm{nm}$ and Keima red at $550 \mathrm{~nm}$. Emission was detected sequentially from 555-620 nm for both excitation wavelengths. Imaging settings were kept constant for all conditions.

\section{RNA live cell imaging}

Rat or mouse hippocampal neurons were seeded in 24-well glass bottom plates (CellVis) at a density of $100^{*} 10^{3}$ and maintained as described above. On DIV5-7, cells were washed three times with NB+PSG and transfected using lipofectamine 2000 transfection reagent (Thermo Fisher) for 20-40 min. After transfection, the original conditioned medium with B27 was returned after three washes. The ideal ratio between the construct encoding the split-Venus parts and the construct encoding the mRNA with the respective binding sites was determined empirically to be approximately 1:4. While we typically observed a high amount of cotransfection in our cultures (around 96\%, compare Wang et al., 2011), only around $10 \%$ of the cells that were transfected with the mitochondrial marker also showed successful fluorophore

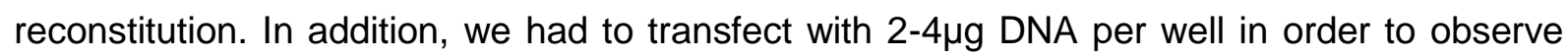
export of the construct from the nucleus. Constructs were expressed for 1-2 days, except in the case of cells cotransfected with shRNA, which were imaged after 3 days to provide enough time for effective reduction of the protein. Imaging was performed in Hibernate $E$ with a spinning disk microscope; using either a Yokogawa CSU-X1 (Olympus IX81) equipped with an electron-multiplying charge-coupled device camera (Andor iXon; Oxford instruments) using a 40×/NA 1.3 or 60×/NA 1.2 oil immersion lens and Metamorph software (Molecular Devices), or a Eclipse Ti2 spinning disk microscope (Nikon) equipped with a DS-Qi2 high-sensitivity monbochrome camera (Nikon) using a 60×/NA 1.2 oil immersion lens and NIS-elements software (Nikon). For puromycin treatment, puromycin was added at a concentration of 200 $\mu \mathrm{g} / \mathrm{mL}$ to the medium $1 \mathrm{~h}$ prior to imaging. 
COS-7 cells were maintained in DMEM + GlutaMax supplemented with penicillin/streptomycin (Life Technologies), and 10\% FBS (Atlanta Premium) and transfected and imaged as described for neurons. Expression of the IRES construct was not as efficient as the P2A split Venus construct and we therefore used the P2A construct for the experiments in COS-7 cells.

\section{In situ hybridization and immunocytochemistry}

RNAscope hybridization was performed as described in Cosker et al., 2016. Briefly, mouse hippocampal neurons were grown on glass coverslips and fixed on DIV7 with $4 \%$ paraformaldehyde for $15 \mathrm{~min}$. After dehydration in a dilution series of ethanol, cells were stored at $-20^{\circ} \mathrm{C}$ for up to one month. Cells were rehydrated and permeabilized with $0.1 \%$ Tween 20/PBS for $10 \mathrm{~min}$, rinsed in PBS and incubated in a 1:5 dilution of Protease III (ACD) for 1020 min at $40^{\circ} \mathrm{C}$ in a preheated hybridization oven. In situ hybridization with mouse PNK1 probes (ACD) was performed at $40{ }^{\circ} \mathrm{C}$ for $2 \mathrm{~h}$ and the detection reactions were performed according to the manufacturer's instructions.

For immunodetection, coverslips were rinsed with PBS and blocked with PBS/0.3\% Triton $\mathrm{X} / 4 \%$ goat serum for $1 \mathrm{~h}$ at RT. Samples were incubated with primary antibodies for 2-3 hours at RT, washed twice fast and twice for $10 \mathrm{~min}$ at $\mathrm{RT}$ with PBS $/ 0.3 \%$ Triton $\mathrm{X}$ before incubation with Alexa488 or Alexa647-conjugated secondary antibodies in PBS/0.3\% Triton $\mathrm{X}$ for $2 \mathrm{~h}$ at RT. After washing in PBS/0.3\% Triton X coverslips were mounted in Fluoromount G (Southern Biotech) and imaged at a confocal microscope (LSM710, Carl Zeiss) using a 63x/NA 1.4 oil immersion objective and ZEN 2009 software (Carl Zeiss) or at a widefield microscope (EVOS M5000, Thermo Fisher) using a 10x objective.

STED imaging was perfomed on RNAscope samples using the Opal570 (RNAscope) and Abberior635P (Immunostaining) fluororescent probes using the same protocol and imaged at a Stedycon system (Abberior) mounted an a Leica DMRXA2 body, using a 100x/NA 1.4 Oil immersion objective and a $775 \mathrm{~nm}$ STED laser.

\section{Kaede photoconversion}

Hippocampal neurons were seeded in 24-well glass bottom plates at a density of $100^{*} 10^{3}$. On DIV7, cells were transfected with PINK1-N-Kaede or mito-Kaede using lipofectamine 2000 transfection reagent for $20 \mathrm{~min}$. Constructs were expressed for $48 \mathrm{hrs}$ to provide enough time for effective expression of the protein. Imaging was performed in Hibernate $E$ with a WF2 Leica Thunder microscope using a HC PL APO 63x/1.20 WATER UV objective and LAS X software. Prior to photoconversion, a defined region of the axon containing Kaede-green fluorescent mitochondria was imaged using 488 and $558 \mathrm{~nm}$ multicolor-illumination. Immediately thereafter, the Kaede-green mitochondria were photoconverted to Kaede-red by using a 405 $\mathrm{nm}$ laser scanner while visually assessing for residual green fluorescence. The axonal regions 
were re-imaged using 488 and $558 \mathrm{~nm}$ multicolor-illumination directly after and 45-60 min postphotoconversion. Only Kaede-red mitochondria, which were still within the defined region, were used for analysis.

\section{Lentiviral transduction}

Lentiviral particles were produced in HEK293T cells as described previously (Pekkurnaz et al., 2014). Hippocampal neurons were transduced on DIV1 or 2 and lysed for Western Blot analysis after 4 days. Infection rates were $60-90 \%$. Western blotting was performed using standard procedures and blots were decorated with the following antibodies diluted in PBS + 5\% milk: Mouse anti-beta-actin Monoclonal Antibody (AC-74) (1:1000, Sigma), Mouse monoclonal anti-Glyceraldehyde-3-PDH (GAPDH) antibody (1:1000, EMD Millipore), Rabbit Polyclonal Anti-CLUH Antibody (1:250, Origene), Rabbit polyclonal anti-SYNJ2BP antibody (1:200, Proteintech). Western Blot analysis was performed using LI-COR secondary antibodies and an Odyssey CLx Infrared Imaging System (LI-COR Biosciences).

\section{RNA isolation and qRT-PCR}

For analysis of RNA abundance after CLUH knockdown, cells were harvested 4 days after lentiviral transduction and RNA was isolated using the QIAGEN RNeasy Mini Kit. cDNA was generated using the qScript ${ }^{\mathrm{TM}}$ cDNA SuperMix (Quantabio) and a qPCR assay was performed using PerfeCTa SYBR® Green FastMix (Quantabio) in a StepOnePlus Real PCR machine (Thermo Fisher). Abundance was calculated relative to beta actin and control shRNA using comparative $\mathrm{Ct}$ using the formula: $2^{-\Delta \Delta C t}$ (relative quantitation) from 3 independent biological repeats.

For analysis of axonal transcripts, rat hippocampal neurons were grown in microfluidic devices. On DIV7 the axonal and soma chamber were lysed individually and RNA was isolated using the QIAGEN RNeasy Mini Kit. cDNA was generated using the qScript ${ }^{\mathrm{TM}}$ cDNA SuperMix (Quantabio) and a qPCR assay was performed using PerfeCTa SYBR $®$ Green FastMix (Quantabio) in a StepOnePlus Real PCR machine (Thermo Fisher). Abundance was calculated relative to mitochondrial S12 rRNA and the soma chamber using comparative $\mathrm{Ct}$ using the formula: $2^{-\Delta \Delta C t}$ (relative quantitation) from 3 independent biological repeats.

For comparison of expression of SYNJ2a and RRBP1 in hippocampal neurons and fibroblasts, DIV7-9 neurons and low passage primary fibroblasts were harvested and RNA was isolated using the QIAGEN RNeasy Mini Kit. cDNA was generated using the qScript ${ }^{\mathrm{TM}}$ cDNA SuperMix (Quantabio) and a qPCR assay was performed using PerfeCTa SYBR $®$ Green FastMix (Quantabio) in a StepOnePlus Real PCR machine (Thermo Fisher). Abundance was calculated relative to beta actin using standard curves generated from beta actin, SYNJ2a and RRBP1 constructs (absolute quantitation), using three to five independent samples. 


\section{Retinal AAV-injection and RNA isolation}

Surgical procedures were performed as described in Park et al. (2008). Mice were anaesthetized with ketamine and xylazine and, to protect the cornea during surgery, eye ointment containing atropine sulfate was applied. A glass micropipette was inserted at an angle posterior to the ora serrata to avoid damage to the lens. $1 \mu$ AAV2-GFP and AAV2-PINK112xMBS-PBS of similar titers were injected intravitreally. As post-operative analgesic mice received Buprenorphine $(0.1 \mathrm{mg} / \mathrm{kg}$, Bedford lab) for $24 \mathrm{hr}$. After four weeks mice were sacrificed and both the retina and the optic nerve prior to the optic chiasm were collected and stored in RNAlater (QIAGEN) at $4{ }^{\circ} \mathrm{C}$ overnight. After removal of RNAlater reagent, $500 \mu \mathrm{l}$ TRIZOL was added and the tissue homogenized on ice using a micropestle mixer. After $5 \mathrm{~min}$ incubation at RT, $100 \mu$ l Chloroform was added and the mixture was vortexed for $15 \mathrm{sec}$ and incubated for $2 \mathrm{~min}$ at RT. Phase separation was achieved during centrifugation at $12000 \mathrm{~g}$ for $15 \mathrm{~min}$ at $4^{\circ} \mathrm{C}$. The aqueous phase was collected and the RNA precipitated with $500 \mu \mathrm{l}$ Isopropanol for $15 \mathrm{~min}$ at RT. RNA was pelleted during a spin at $12000 \mathrm{~g}$ for $15 \mathrm{~min}$, washed with $70 \%$ ethanol, and resuspended in $50 \mu \mathrm{l}$ Rase-free water for retina - $25 \mu$ l for optic nerve samples. cDNA was generated using the qScript ${ }^{\mathrm{TM}}$ cDNA SuperMix (Quantabio) and a qPCR assay was performed using PerfeCTa SYBR® Green FastMix (Quantabio) in a StepOnePlus Real PCR machine (Thermo Fisher). Abundance was calculated relative to beta actin and the retinal amount using comparative $\mathrm{Ct}$ using the formula: $2^{-\Delta \Delta C t}$ (relative quantitation). Four retina/optic nerve pairs were analyzed per transcript.

\section{Cross-Linking Immunoprecipitation (CLIP)}

HEK293T cells were grown in 6 well plates and transfected with $3 \mu \mathrm{g} /$ well Myc-SYNJ2aRRMmito or its VQL/AAA mutant. UV irradiation was performed by washing the cells with PBS and placing the plate on ice in a CL-1000 crosslinker (UVP) and exposing the plate to $400 \mathrm{~mJ} / \mathrm{cm}^{2}$ $254 \mathrm{~nm}$ UV light. After irradiation, cells were harvested in Lysis Buffer (1\% Triton, 20mM Tris $\mathrm{pH} 7.4,200 \mathrm{mM} \mathrm{NaCl}, \mathrm{RNAsin}$ (1:100 Promega), Protease inhibitor cocktail III (Millipore) and $200 \mu \mathrm{M}$ PMSF) and cleared by centrifugation at $12000 \mathrm{~g}$ for $1 \mathrm{~min}$. The supernatant was incubated with $3 \mu \mathrm{l}$ anti-myc antibody (mouse 9E10, Novus) $/ \mathrm{mL}$ lysate for $1 \mathrm{~h}$ at $4^{\circ} \mathrm{C}$. ProteinA sepharose beads were blocked with $3 \%$ BSA in lysis buffer for $30 \mathrm{~min}$, washed with PBS and added to the lysate. After $30 \mathrm{~min}$ incubation at $4^{\circ} \mathrm{C}$ beads were collected by centrifugation at $2000 \mathrm{~g}$ for $30 \mathrm{sec}$ and washed three times with lysis buffer. Samples were eluted by addition of Laemmli Buffer and boiling at $95^{\circ} \mathrm{C}$ for 3 min prior to analysis by gel electrophoresis and immunoblotting with Rabbit myc-tag antibody, 71D10 (Cell Signaling).

\section{Proximity ligation assay (PLA)}


The proximity ligation assay was performed according to the manufacturer's instructions (Sigma-Aldrich). Briefly, primary mouse hippocampal neurons were grown on glass coverslips, fixed on DIV 8 with $4 \%$ paraformaldehyde for $15 \mathrm{~min}$ and permeabilized with $0.3 \%$ Triton/PBS for $10 \mathrm{~min}$ followed by a $1 \mathrm{~h}$ incubation with Duolink blocking solution at $37^{\circ} \mathrm{C}$. Neurons were incubated with primary antibodies (SYNJ2BP-SYNJ2 interaction, SYNJ2BP-ATP5B interaction, SYNJ2-RHOT1 interaction; mouse polyclonal SYNJ2BP antibody, 1:50, SigmaAldrich; rabbit polyclonal SYNJ2 antibody, 1:50, Proteintech; rabbit polyclonal ATP5b antibody, 1:600, Sigma-Aldrich; mouse monoclonal RHOT1 antibody (4H4), 1:500, Sigma-Aldrich) diluted in Duolink antibody diluent at $4^{\circ} \mathrm{C}$ overnight. Neurons were washed two times with Buffer A (0.01 M Tris, $0.15 \mathrm{M} \mathrm{NaCl}$ and $0.05 \%$ Tween 20) at RT for $5 \mathrm{~min}$, incubated with Duolink PLA Probes (Anti-Rabbit Plus and Anti-Mouse Minus) at $37^{\circ} \mathrm{C}$ for $1 \mathrm{~h}$, again washed two times with Buffer $A$ at RT for 5 min, then incubated with Duolink ligation solution at $37^{\circ} \mathrm{C}$ for $30 \mathrm{~min}$, again washed two times with Buffer $A$ at RT for 5 min and incubated with Duolink amplification solution at $37^{\circ} \mathrm{C}$ for $100 \mathrm{~min}$. After two washes with Buffer B (0.2 M Tris, $0.1 \mathrm{M}$ $\mathrm{NaCl}$ ) at RT for 10 min and a final wash with $0.01 x$ Buffer $B$ for 1 min the coverslips were mounted in Fluoromount G (Invitrogen) and imaged at a Nikon Ti2 spinning disk microscope using a 60x/NA 1.40 oil immersion objective. For puromycin treatment, puromycin was added at a concentration of $200 \mu \mathrm{g} / \mathrm{ml}$ to the medium $1 \mathrm{~h}$ prior to fixation. The number of PLA puncta per soma was quantified and normalized to the number of the SYNJ2BP-SYNJ2 PLA puncta.

\section{QUANTIFICATION AND STATISTICAL ANALYSIS, SUPPLEMENTAL INFORMATION}

Throughout the paper, data are expressed as mean \pm SEM. Statistical analysis was performed with Excel (Windows) or $\mathrm{R}$ (The $\mathrm{R}$ foundation) using student's t-test for gaussian distributions. When comparing multiple conditions a one-way ANOVA test for statistical significance was followed up by a Bonferroni post-hoc test. $p<0.05$ was considered significant $\left({ }^{*}\right)$, with further levels defined as $\left.p<0.01{ }^{* *}\right), p<0.001\left(^{* * *}\right)$ and $\left.p<0.0001{ }^{* * * *}\right)$. Where practical, especially for values $>0.01$, actual $p$ values are given in the figure or figure legend.

Quantification of Western Blots was performed in Image Studio Lite (LI-COR) using the local background correction. Quantification of microscopy data was performed using Image J. Colocalization was analyzed in z-stack images using the JaCOP and "straighten" plugins as described in Graber et al. (2013). For dendrites and axons imaged with the split-Venus approach, after maximum z projection neurites were straightened with a $20 \mathrm{px}$ margin. For the cell body quantification, a 10 by $10 \mu \mathrm{m}$ square was chosen within the cell body and no $\mathrm{z}$ projection was performed. The position of the square was chosen based on the mitochondrial signal to exclude the nucleus and blinded to the phenotype of the RNA channel. Mander's correlation coefficients were exported to Excel and plotted in $\mathrm{R}$ using the $\mathrm{R}$ boxplot function. 
Box and whisker plots represent the median (line), 25th-75th percentile (box) and 10th-90th percentile (whiskers).

Time-lapse imaging was performed by imaging every $1 \mathrm{sec}$ for $90 \mathrm{sec}$. Movies were analyzed using Kymolyzer macro for ImageJ developed in the laboratory (Pekkurnaz et al., 2014). Time spent in motion was averaged for every dendrite separately, which creates a gaussian distribution of average time spent in motion per neurite.

For the histogram of length traveled, 43 movies that showed at least one moving PINK1 RNA particle were converted to kymographs and the distance in $\mathrm{x}$ was measured for the intervals of movement. The corresponding mitochondrial kymograph was then examined and, if a similar mitochondrial track was observed, the even was scored as a co-movement.

Keima images were analyzed using Image J. Mitochondrial were identified on a thresholded image using the particle analyzer and the intensities of the non-thresholded images were calculated. For each field of view the mean integrated density was exported to Excel. The mitophagy index was expressed as $\mathrm{in}^{9}$ by calculating the ratio of the integrated density signals [Keima red/(Keima red + Keima green)].

Kaede images were analyzed using Image J. Mitochondrial were identified on a thresholded image using the particle analyzer and the intensities of the non-thresholded images were calculated. The mean intensities as well as a background intensity were exported to Excel and the substracted values were visualized with the R ggplot2 library.

Myc-SYNJ2 ICC images were analyzed using ImageJ. Myc signal was identified on a thresholded image using the particle analyzer and the intensities of the non-thresholded images were calculated. The mean integrated densities of the myc signal and the mitomRaspberry signal were exported to Excel. For each neuron, the mean integrated density of the myc signal was normalized to the mean integrated density of the mito-mRaspberry signal. HA-splitVenus ICC images were analyzed using ImageJ. Axons were traced using the segmented line tool for each field of view and the mean integrated densities of the HA and iRFP signals were measured and exported to Excel. The HA intensity was normalized to the iRFP intensity. The first two fields of view are classified as "proximal", whereas all subsequent fields of view were categorized as distal as they are more than $750 \mu \mathrm{m}$ away from the cell body.

\section{Additional References}

Cartoni R, Norsworthy MW, Bei F, Wang C, Li S, Zhang Y, et al. (2016) The MammalianSpecific Protein Armcx1 Regulates Mitochondrial Transport during Axon Regeneration. Neuron. 92, 1294-1307.

Cosker, K.E., Fenstermacher, S.J., Pazyra-Murphy, M.F., Elliott, H.L., and Segal, R.A. (2016). The RNA-binding protein SFPQ orchestrates an RNA regulon to promote axon viability. Nat. Neurosci. 19, 690-696.

Friedman JR, Lackner LL, West M, DiBenedetto JR, Nunnari J, Voeltz GK (2011) ER tubules mark sites of mitochondrial division. Science 334(6054), 358-62. 
Gibson DG, Young L, Chuang R-Y, Venter JC, Hutchison CA, Smith HO. (2009) Enzymatic assembly of DNA molecules up to several hundred kilobases. Nat. Methods. 6, 343345.

Graber TE, Hébert-Seropian S, Khoutorsky A, David A, Yewdell JW, Lacaille J-C, et al. (2013) Reactivation of stalled polyribosomes in synaptic plasticity. Proc. Natl. Acad. Sci. U. S. A.. 110, 16205-10.

Hung V, Lam SS, Udeshi ND, Svinkina T, Guzman G, Mootha VK, Carr SA, Ting AY. (2017) Proteomic mapping of cytosol-facing outer mitochondrial and ER membranes in living human cells by proximity biotinylation. Elife. 2017 Apr 25;6

Leterrier C, Vacher H, Fache MP, d'Ortoli SA, Castets F, Autillo-Touati A, Dargent B. (2011) End-binding proteins EB3 and EB1 link microtubules to ankyrin $G$ in the axon initial segment. Proc Natl Acad Sci U S A. 108(21), 8826-31.

Nemoto Y, Arribas M, Haffner C, DeCamilli P. (1997) Synaptojanin 2, a novel synaptojanin isoform with a distinct targeting domain and expression pattern. J. Biol. Chem. [Internet]. 272, 30817-21.

Park K, Liu K, Hu Y, Smith P, Wang C, Cai B, et al. (2008) Promoting Axon Regeneration in the Adult CNS by Modulation of the PTEN/mTOR Pathway. Science. 322, 16731675.

Pekkurnaz G, Trinidad JC, Wang X, Kong D, Schwarz TL. (2014) Glucose Regulates Mitochondrial Motility via Milton Modification by O-GlcNAc Transferase. Cell. 158, 5468.

Schindelin et al. (2015) Schindelin J, Rueden CT, Hiner MC, Eliceiri KW. The ImageJ ecosystem: an open platform for biomedical image analysis. Molecular Reproduction and Development. 2015;82(7-8):518-529.

Shlevkov E, Kramer T, Schapansky J, LaVoie MJ, Schwarz TL. (2016) Miro phosphorylation sites regulate Parkin recruitment and mitochondrial motility. Proc. Natl. Acad. Sci. [Internet]. 113, E6097-E6106.

van den Ent F, Löwe J. (2006) RF cloning: a restriction-free method for inserting target genes into plasmids. J. Biochem. Biophys. Methods. 67, 67-74.

Vargas JNS, Wang C, Bunker E, Hao L, Maric D, Schiavo G, Randow F, Youle RJ (2019) Spatiotemporal Control of ULK1 Activation by NDP52 and TBK1 during Selective Autophagy. Mol Cell. 2019 Apr 18;74(2):347-362.e6.

Wang X, Winter D, Ashrafi G, Schlehe J, Wong YL, Selkoe D, et al. (2011) PINK1 and Parkin target Miro for phosphorylation and degradation to arrest mitochondrial motility. Cell. 147, 893-906.

Willis D, Li KW, Zheng J-Q, Chang JH, Smit AB, Smit A, et al. (2005) Differential transport and local translation of cytoskeletal, injury-response, and neurodegeneration protein mRNAs in axons. J. Neurosci. 25, 778-91. 
A

CHX pretreatment $\frac{\text { Control }}{-}+\frac{\text { CCCP }}{-}++$

PINK1 -

GAPDH

$37 \mathrm{kDa}$

B

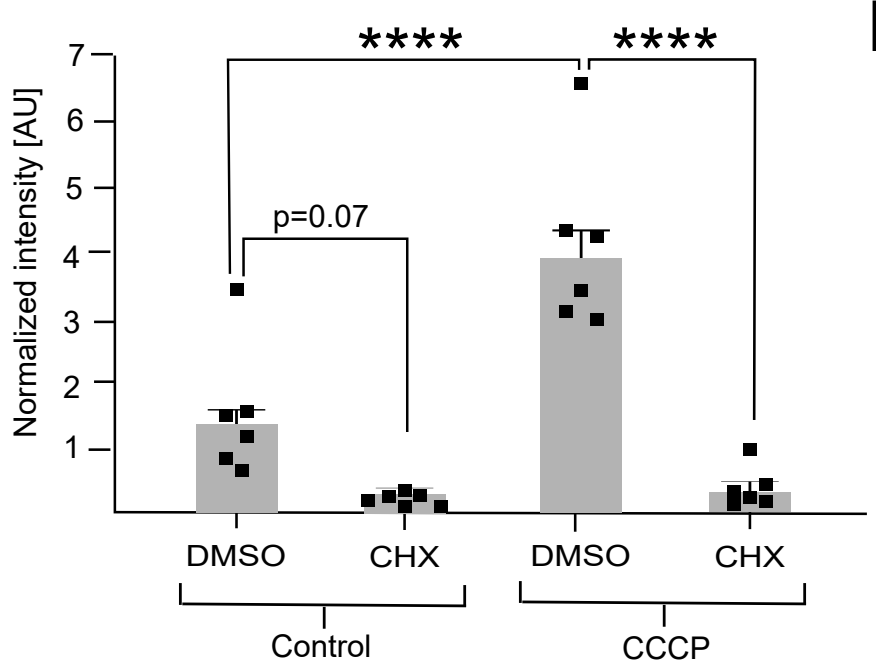

E

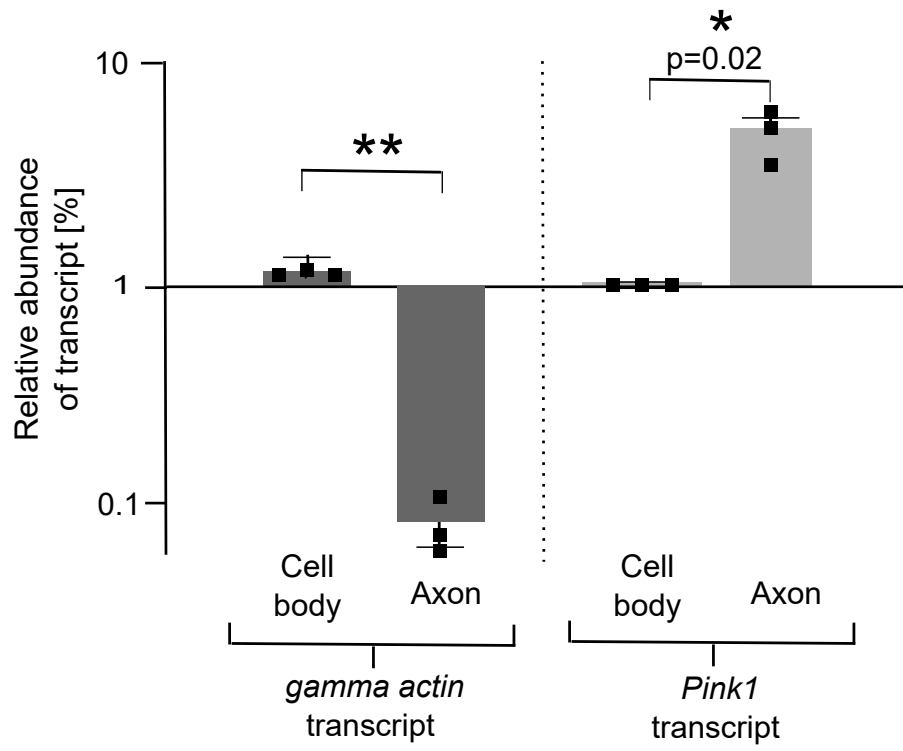

F

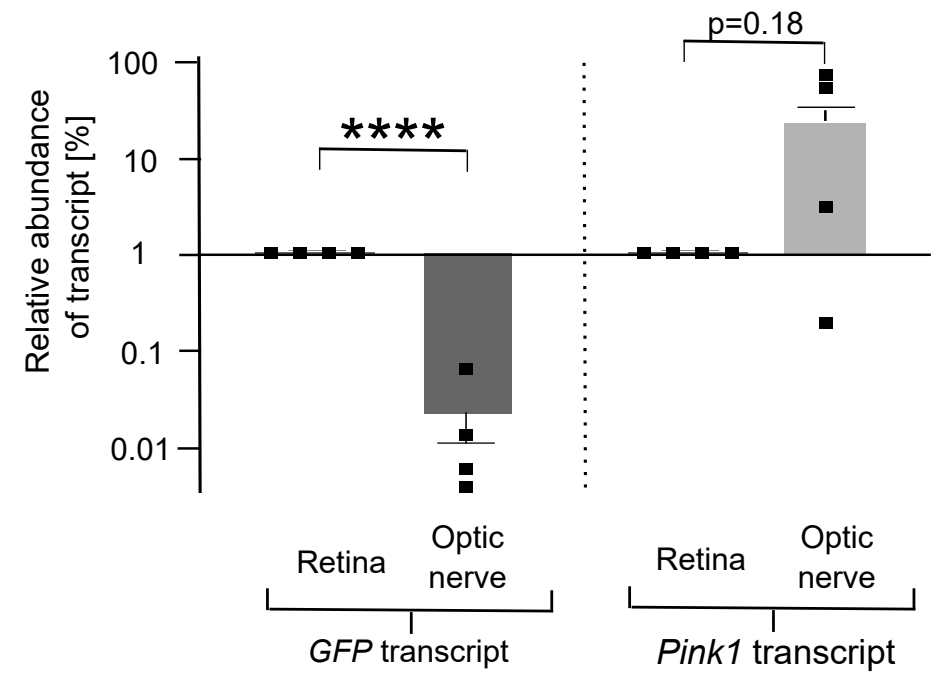

D
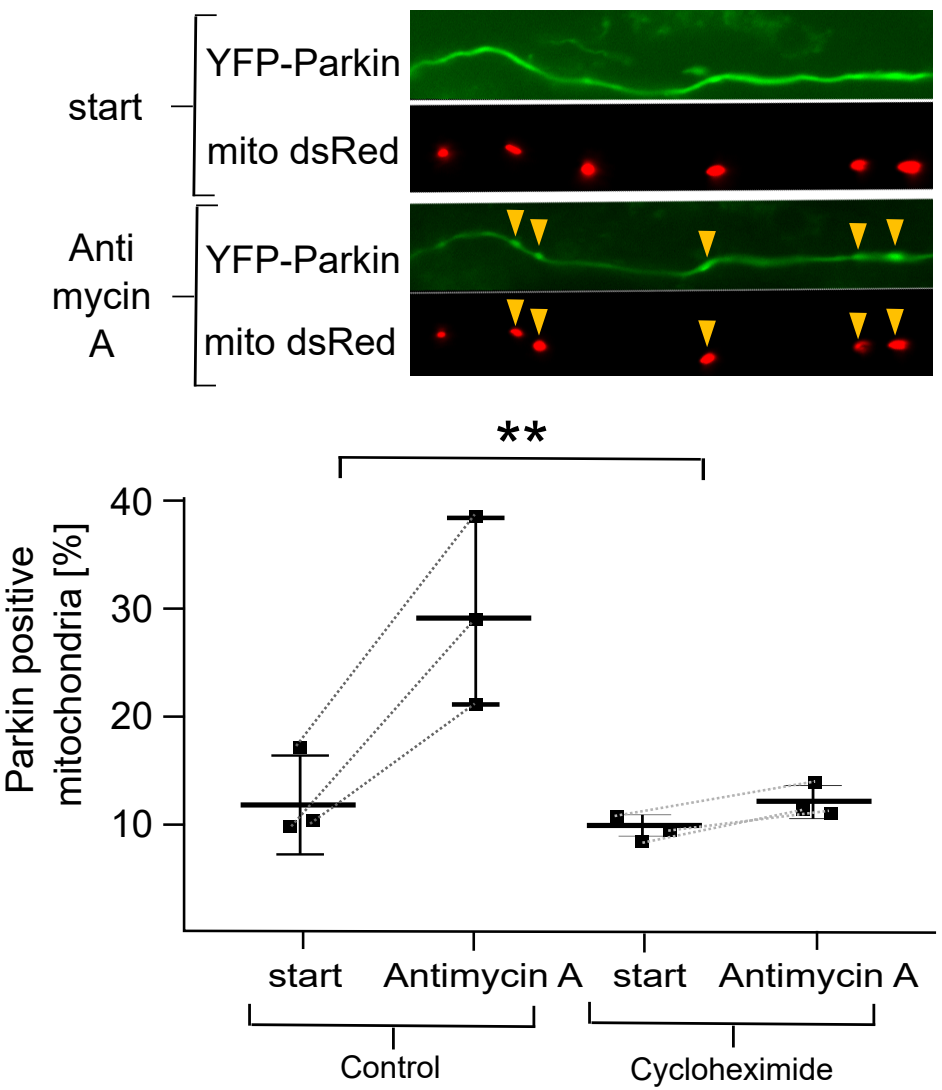

G

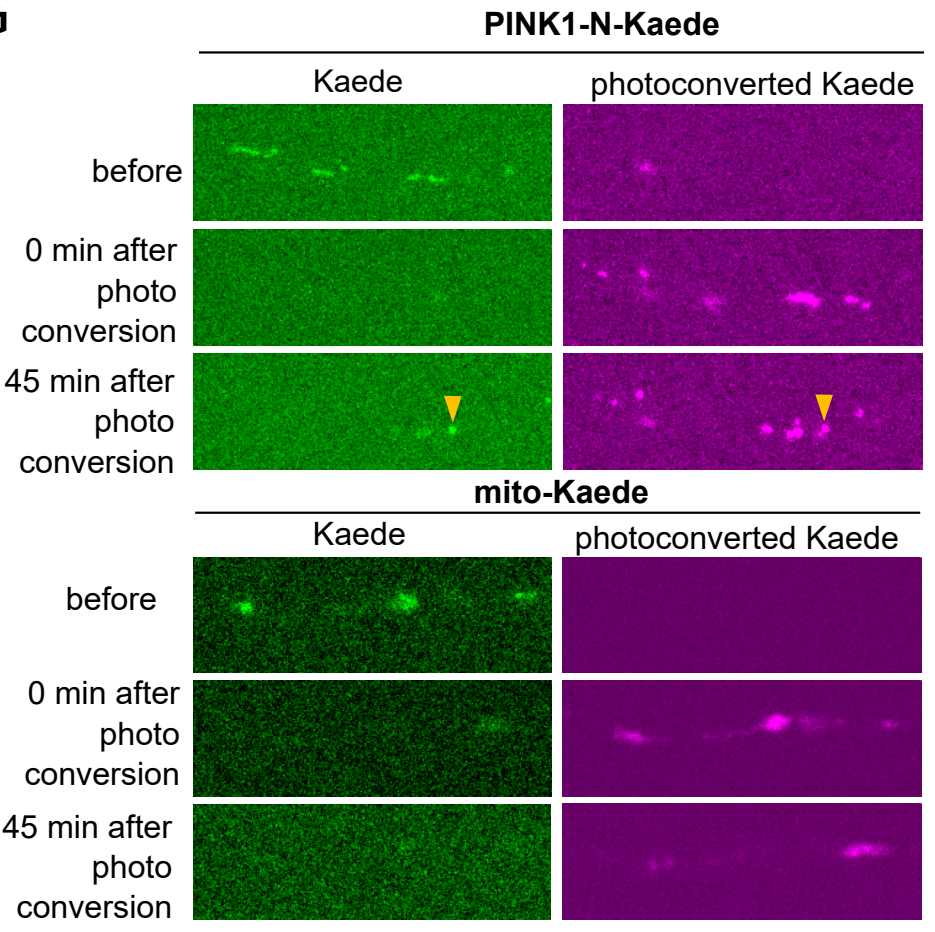

$\mathrm{H}$

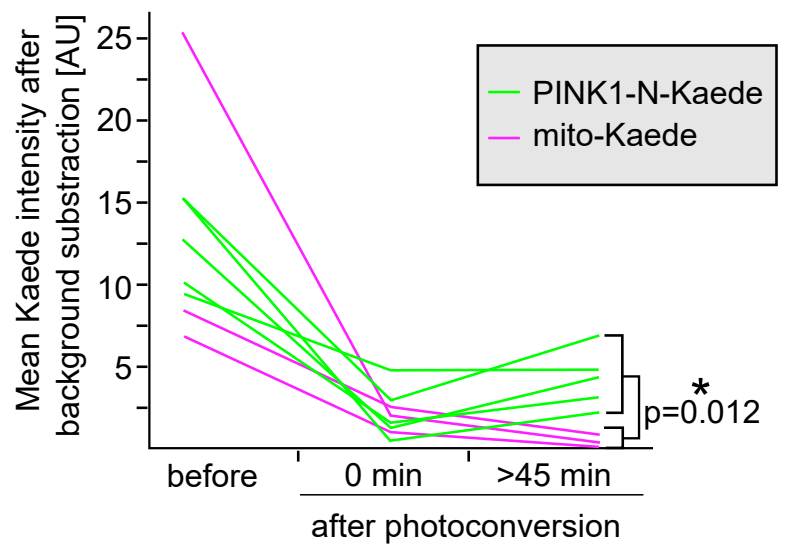


A

Axon - fixed cells

Pink1 mRNA RNAscope

Mitochondria ATP5a ICC

Pink1 mRNA Mitochondria

B

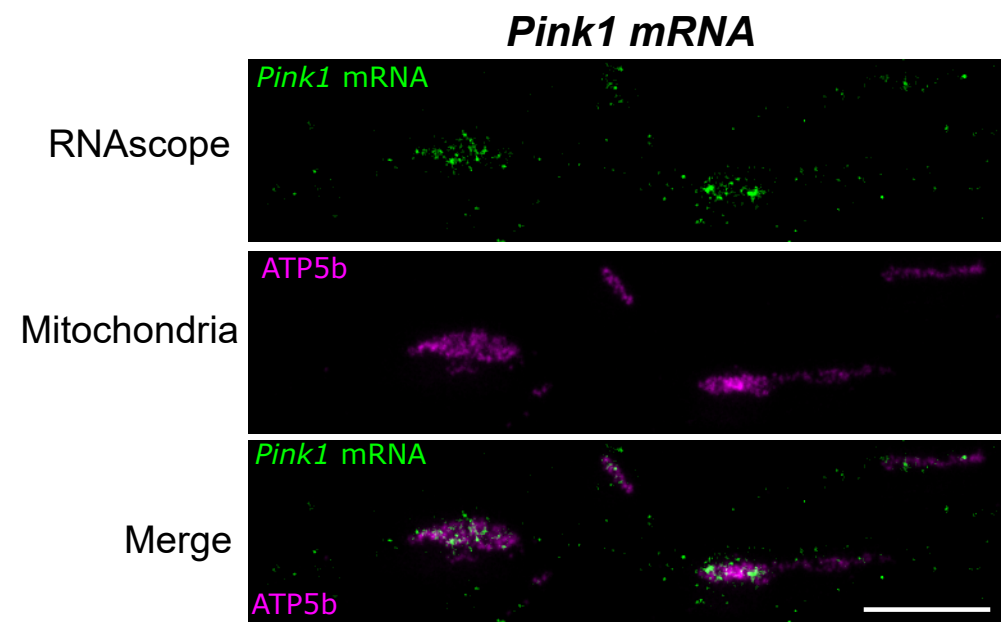

\section{Pink1 mRNA}

C

Venus $_{\mathrm{N}}-\mathrm{MS} 2$ coat protein

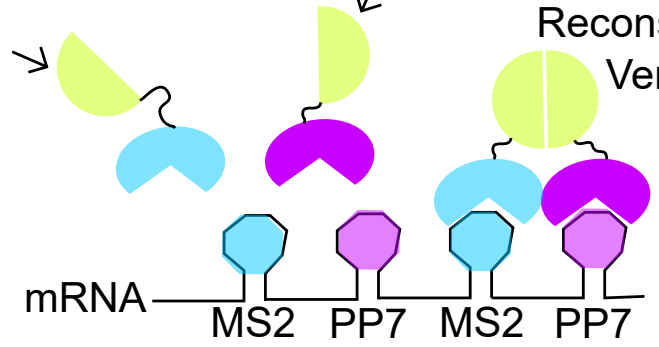

D

Pink1 mRNA

tituted Venus
E

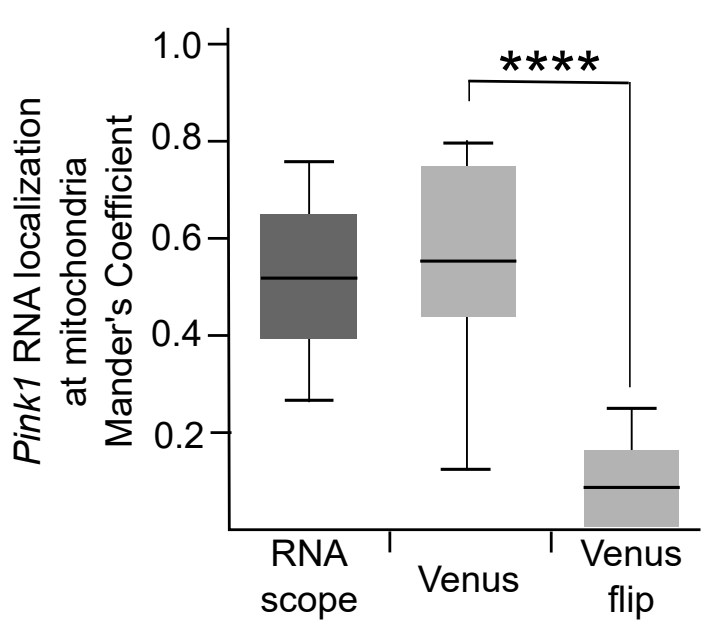

F

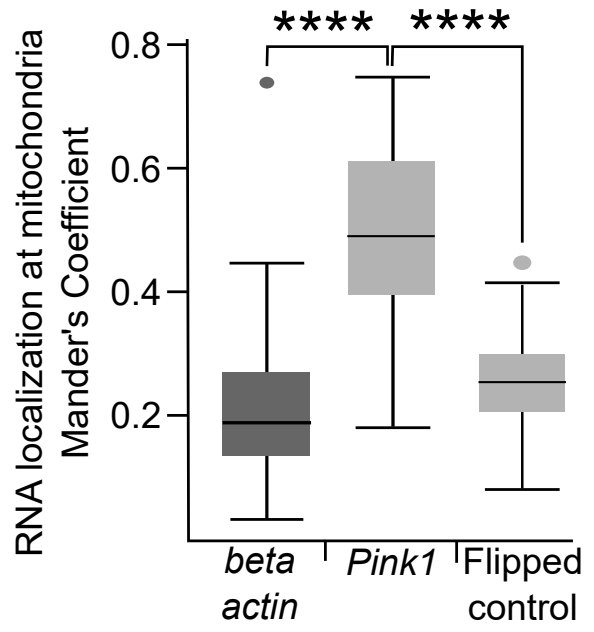

Axon - live cell imaging

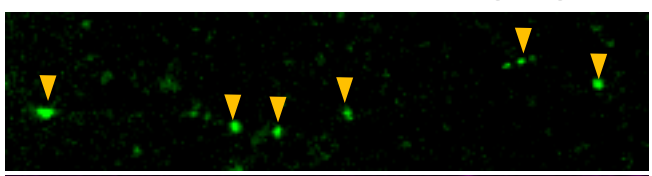

mitoBFP

Pink1 mRNA mitoBFP

mitoB

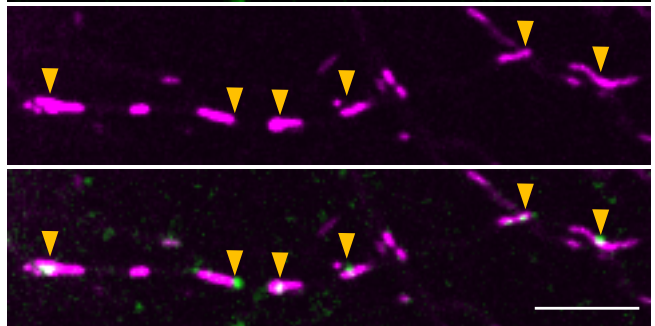

beta actin $m R N A$

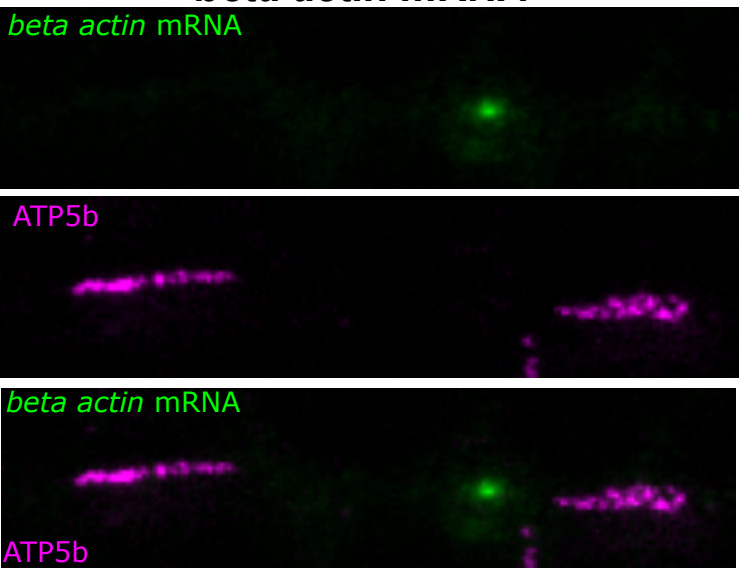

Dendrite - fixed cells

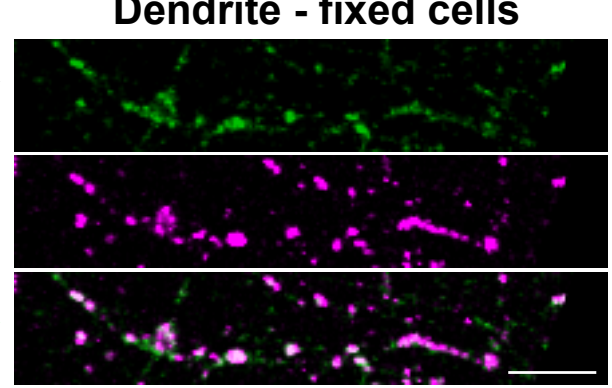

Mitochondria

Pink1 mRNA

Mitochondria

Pink1 mRNA

RNAscope

ATP5a ICC

Cellbody

G

Dendrite

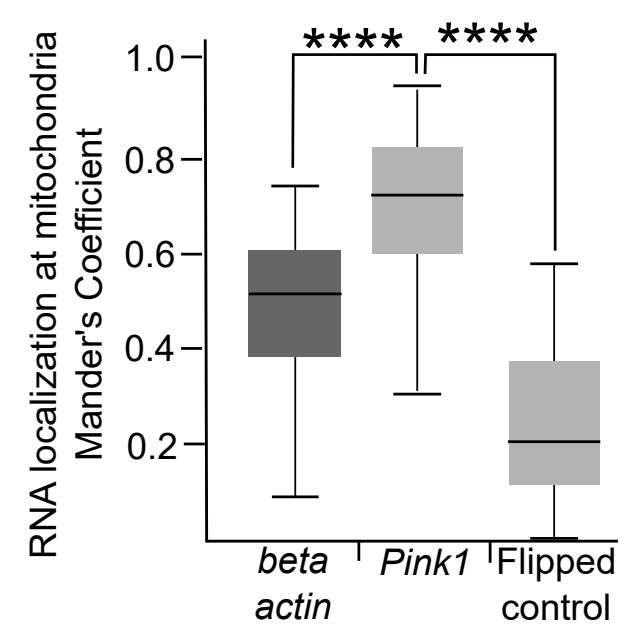


A Dendrite - live cell imaging B

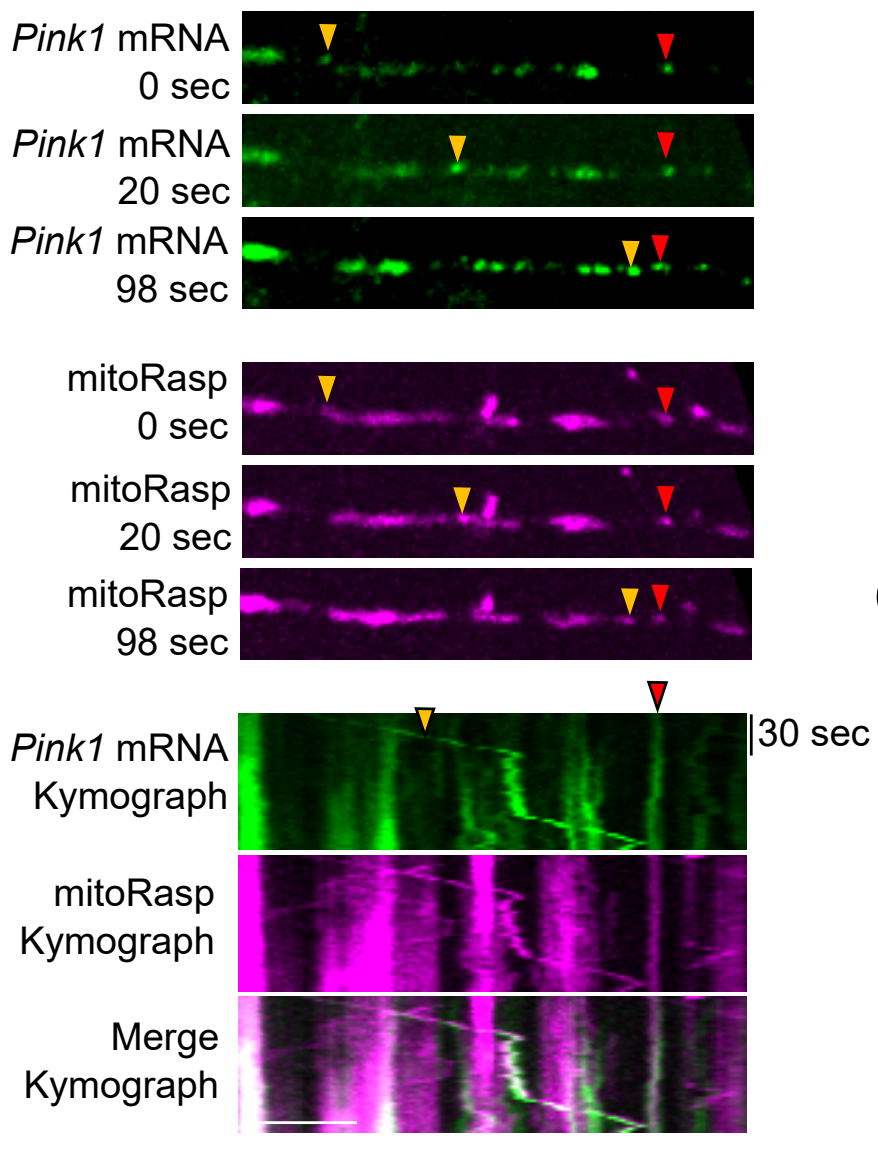

Dendrite - live cell imaging
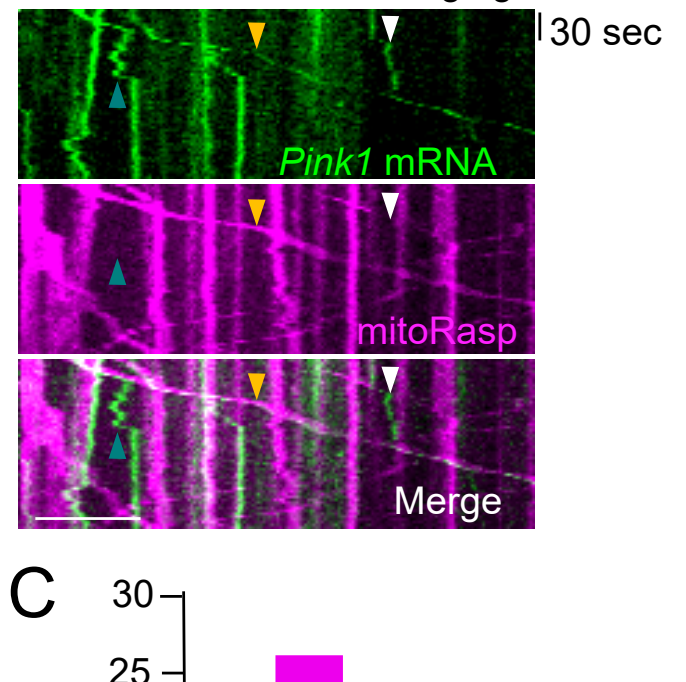

D

$30 \mathrm{sec}$

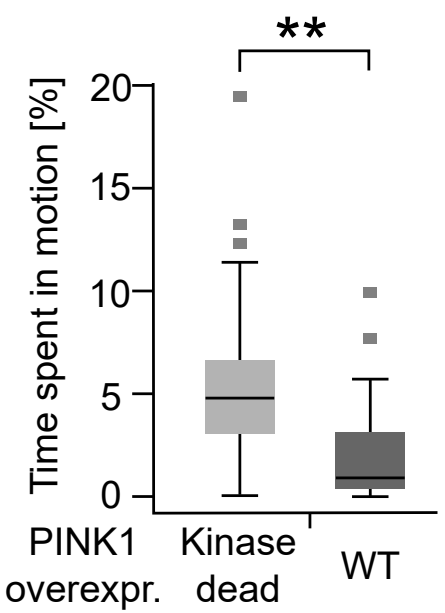

Pink1 mRNA movement

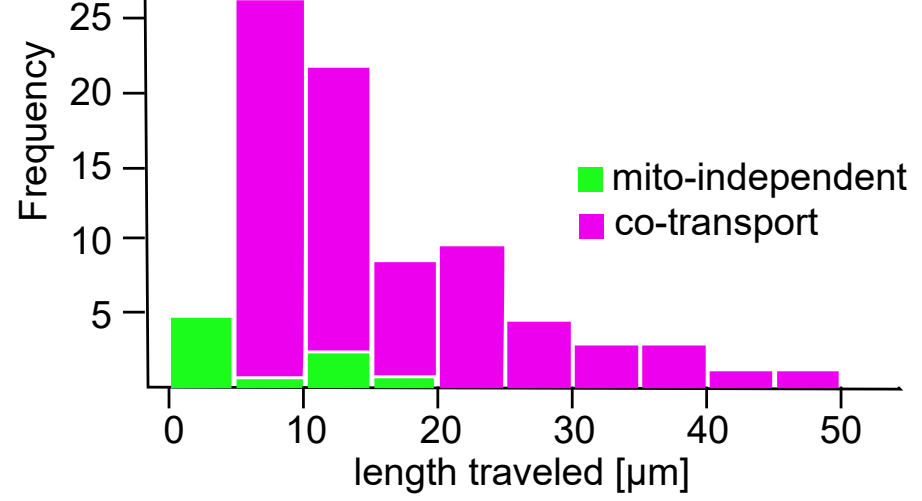


A

mRNA localization at mitochondria
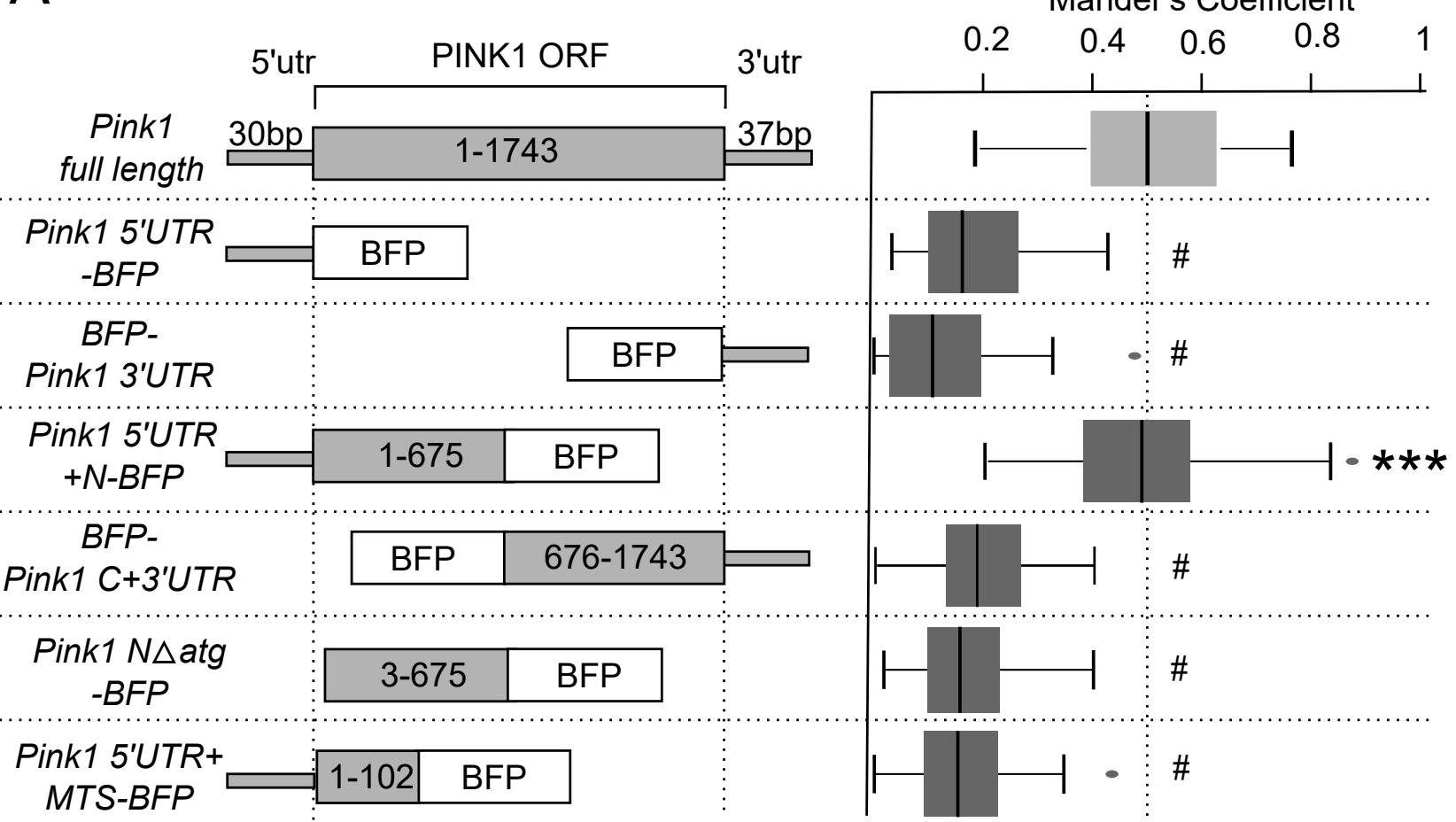

B
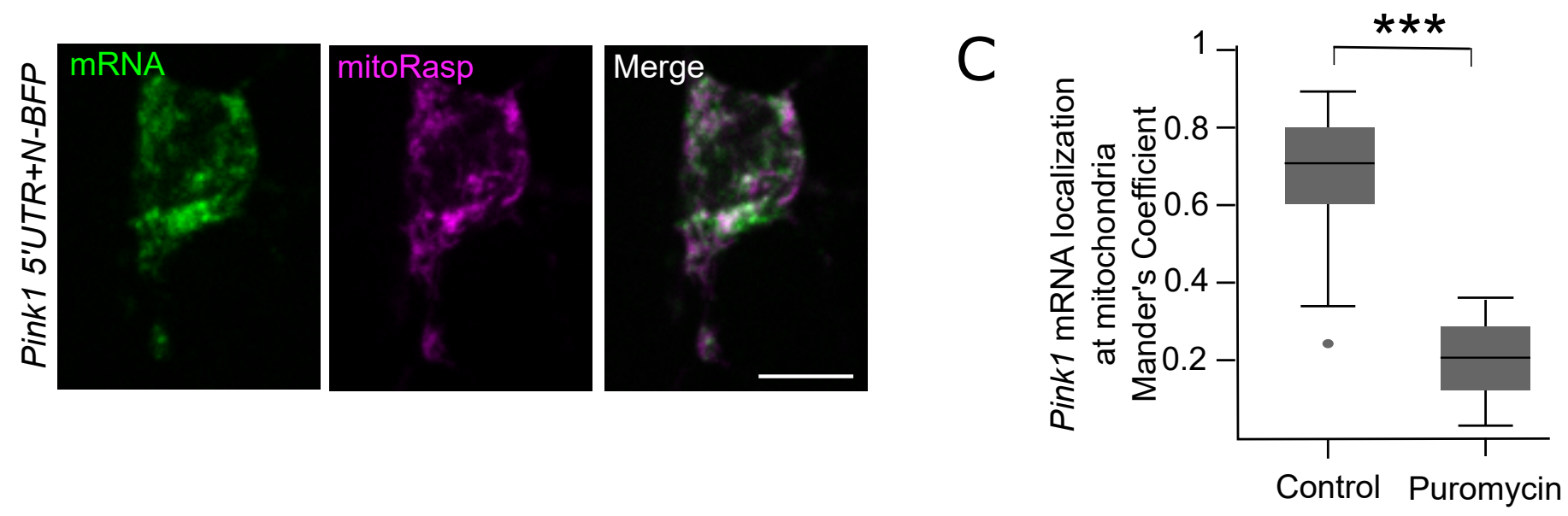

D
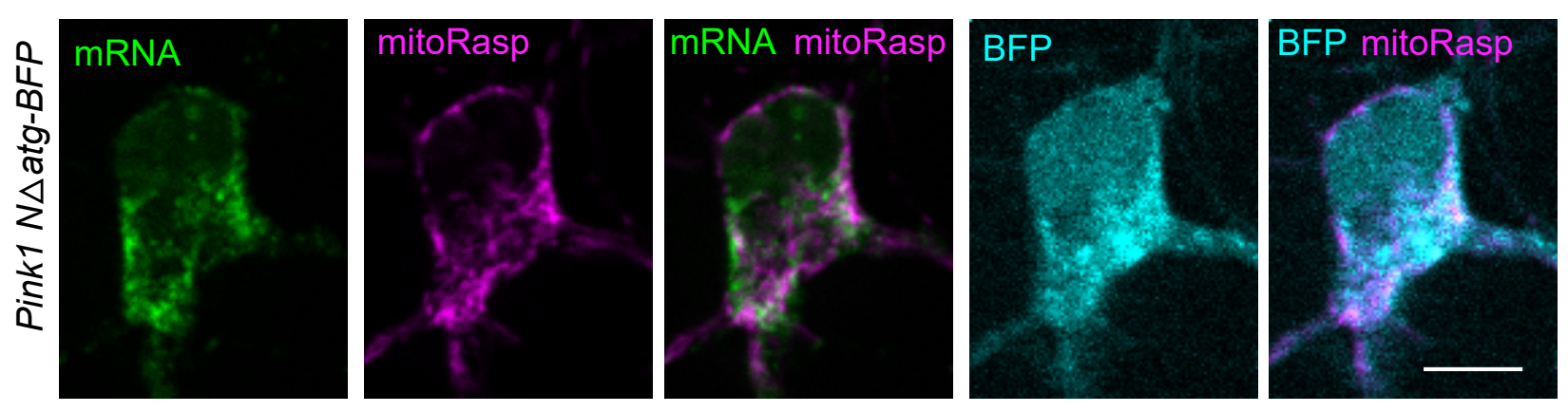

E
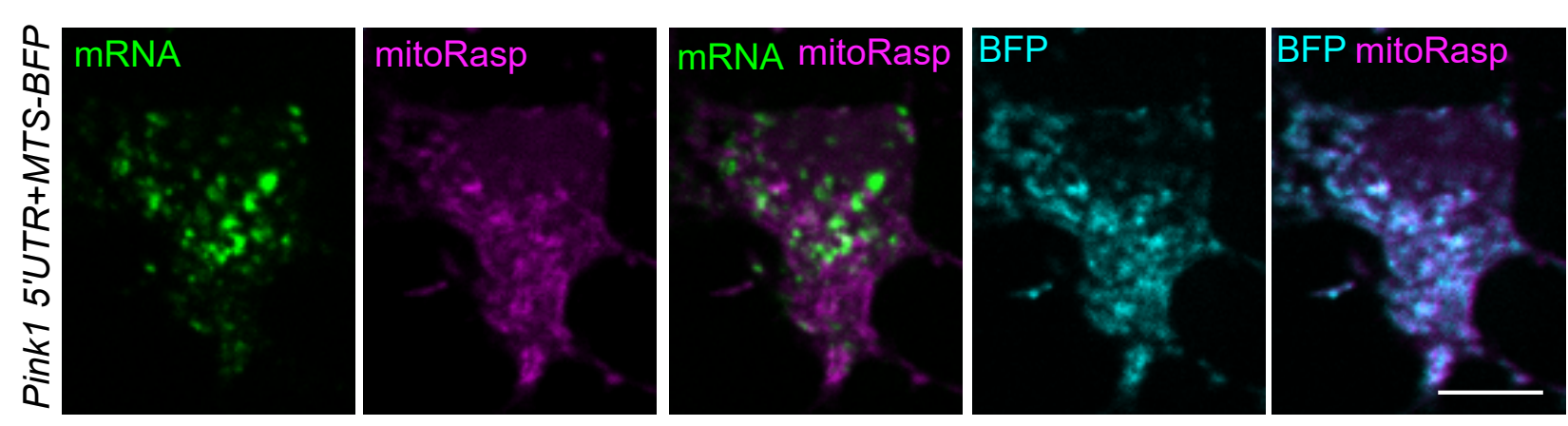
A
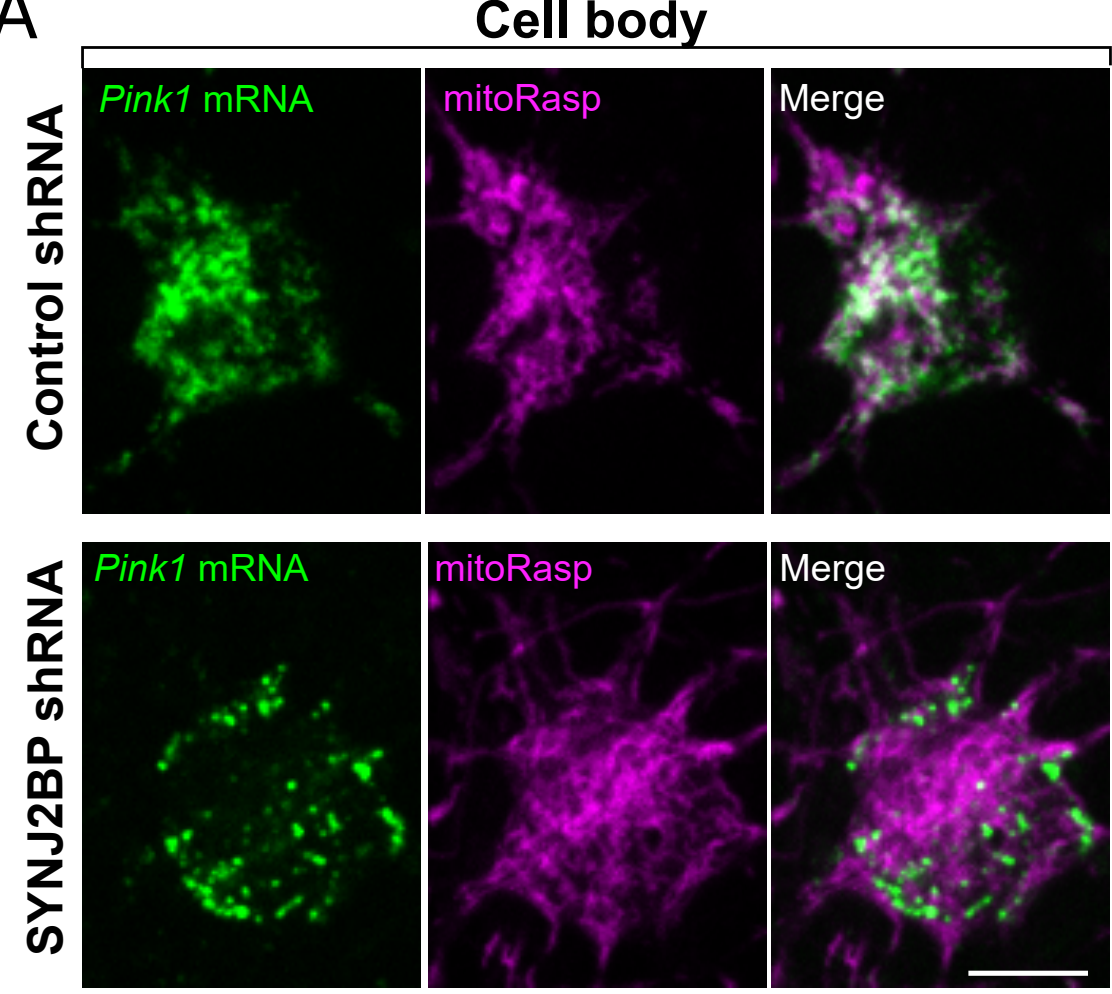

B

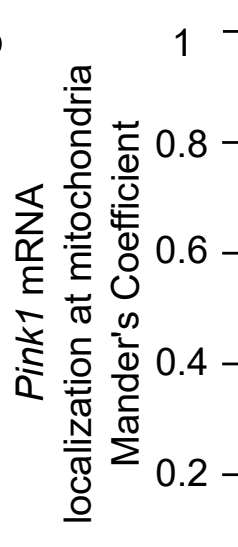

shRNA

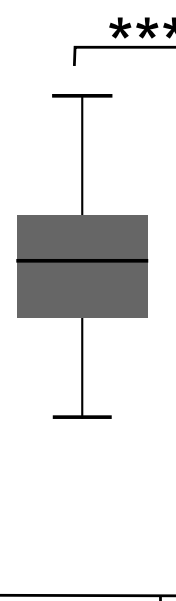

Contro

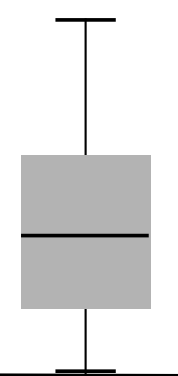

SYN

\section{Overexpression}

$D$

\section{Control shRNA}

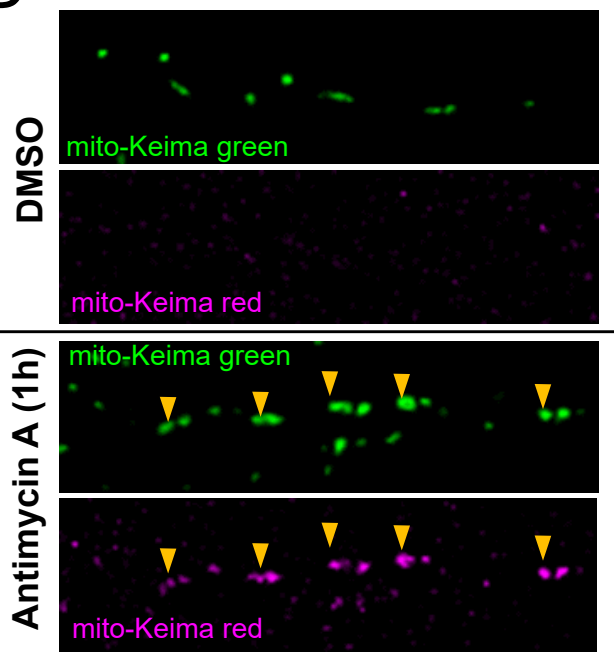

○

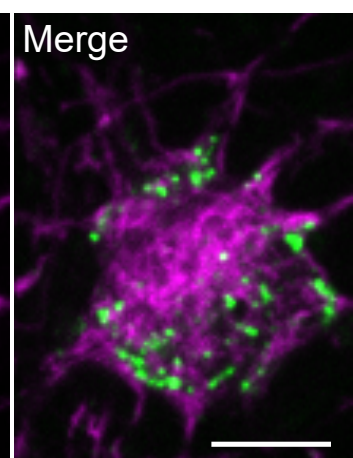

$\sum_{\alpha}$

$\frac{\varepsilon}{\omega}$

ก

$\frac{\mathbf{N}}{2}$

ஸ Merge

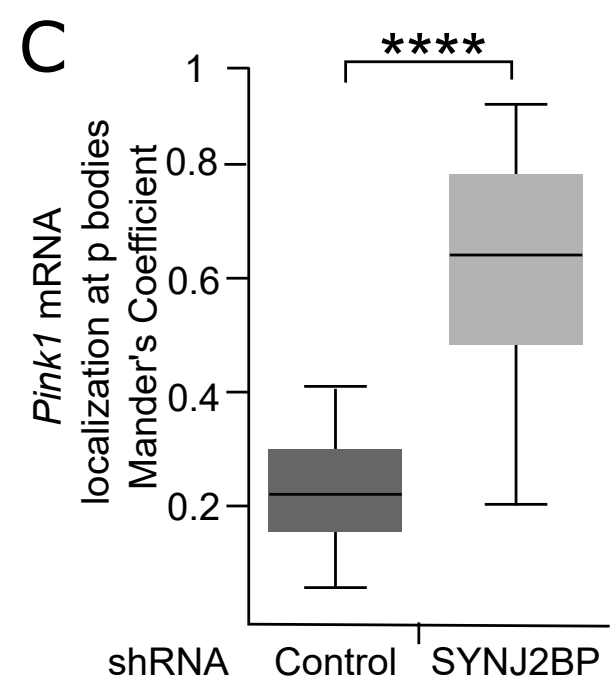

resistant

SYNJ2BP
Pink1 mRNA

mitoRasp

Dendrite

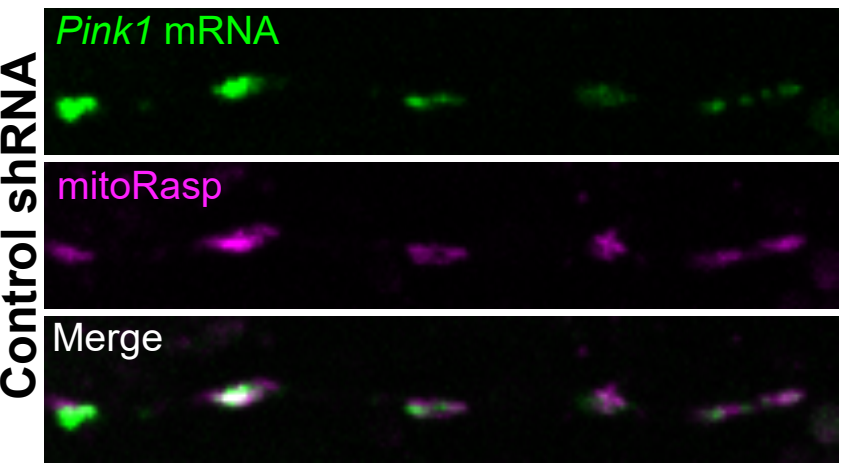

$\mathrm{M}$

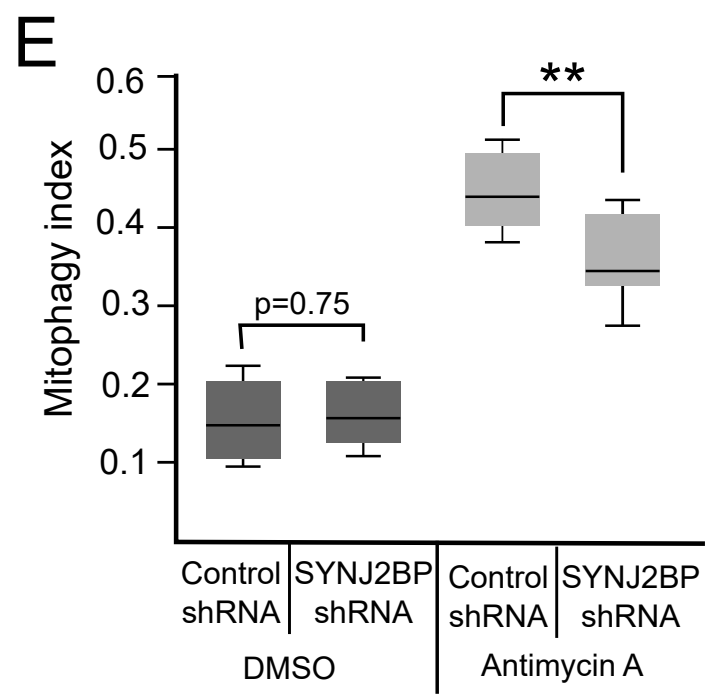




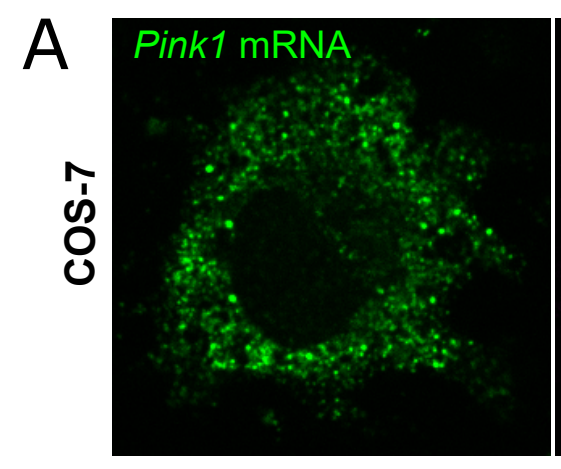

B

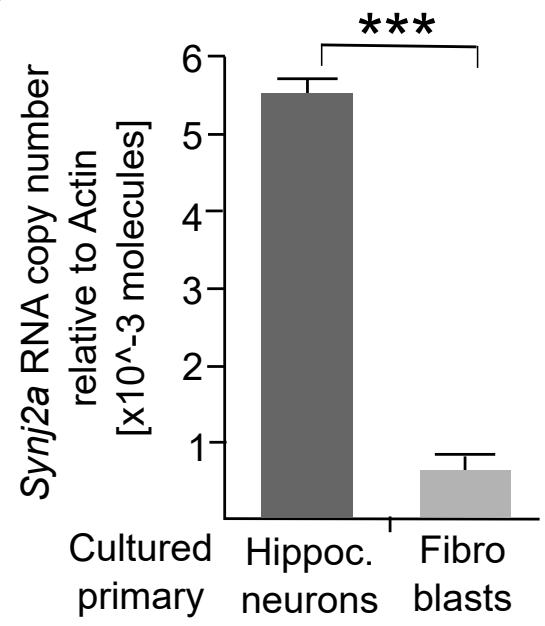

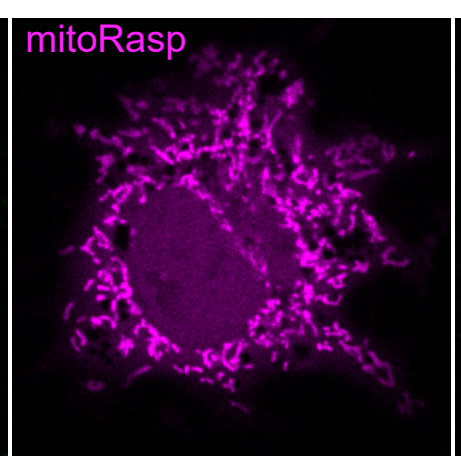

C

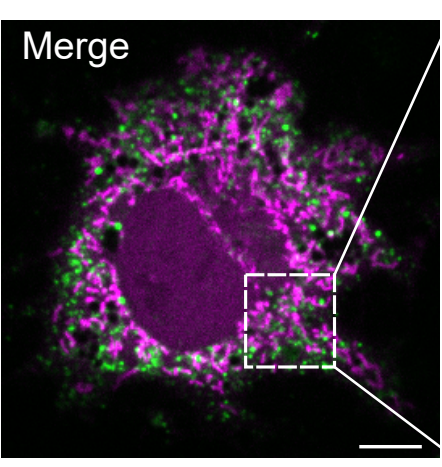

D
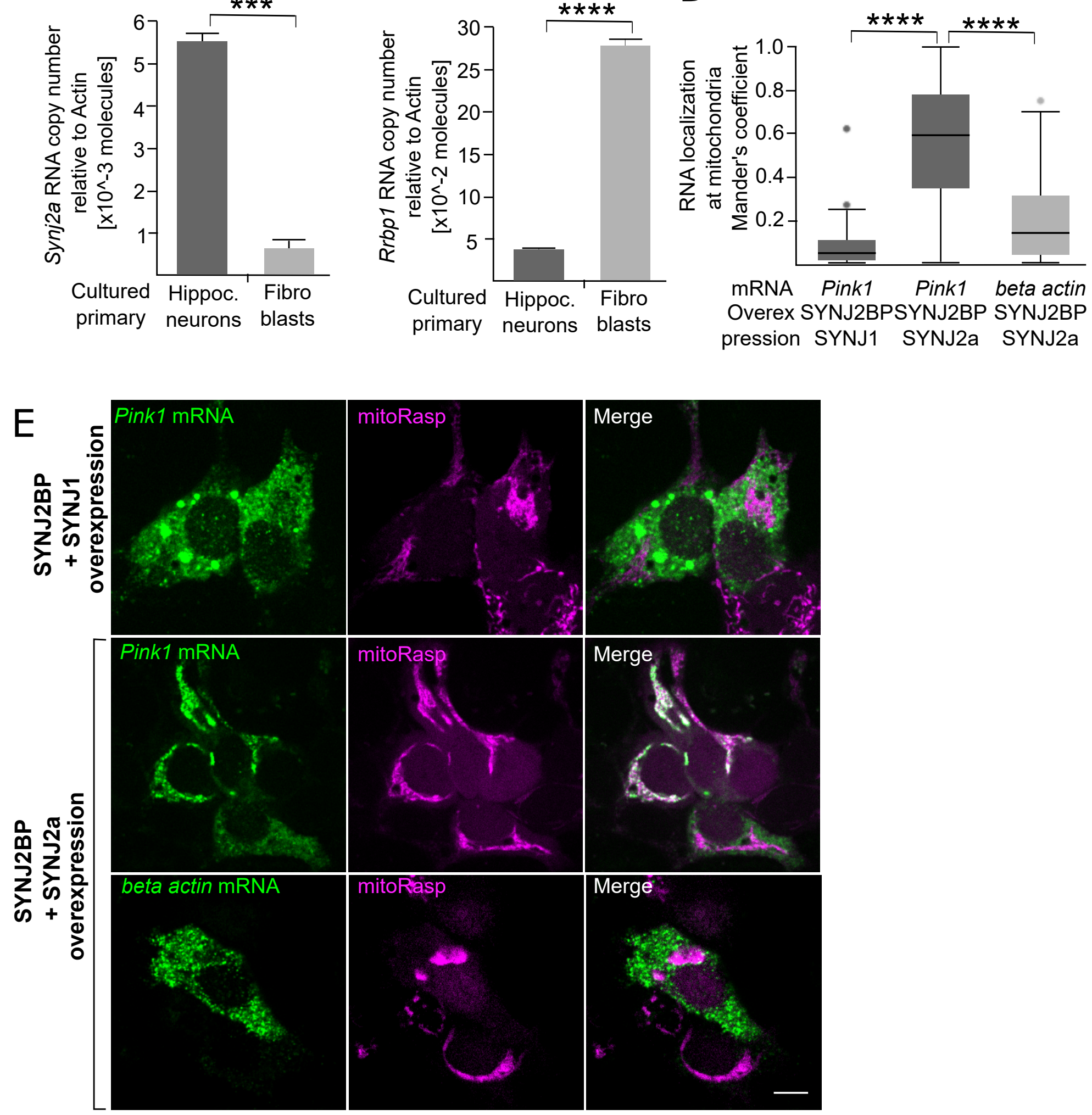
A

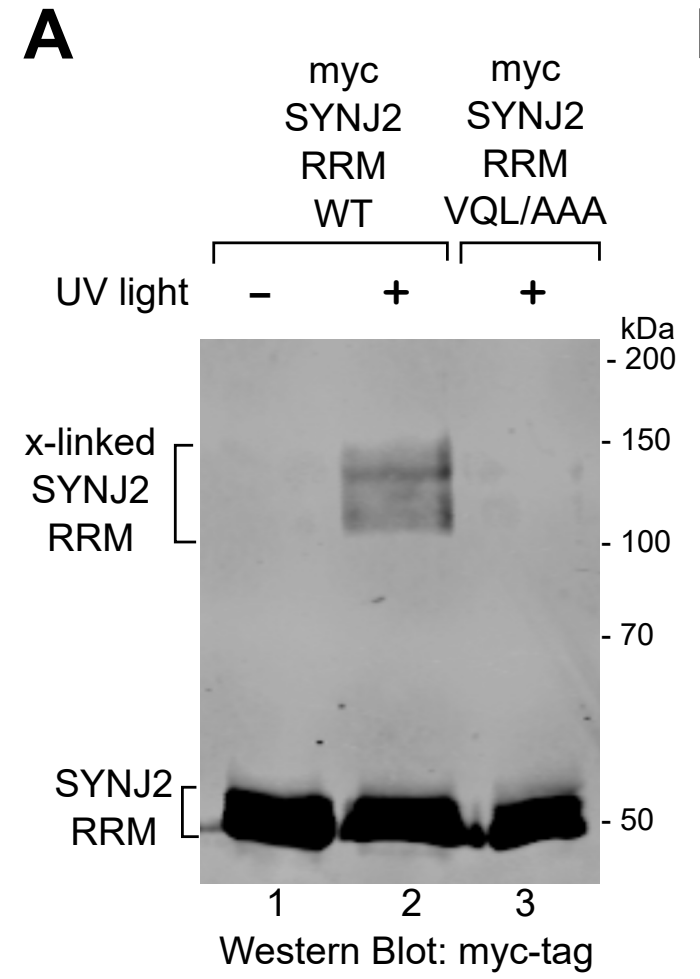

B
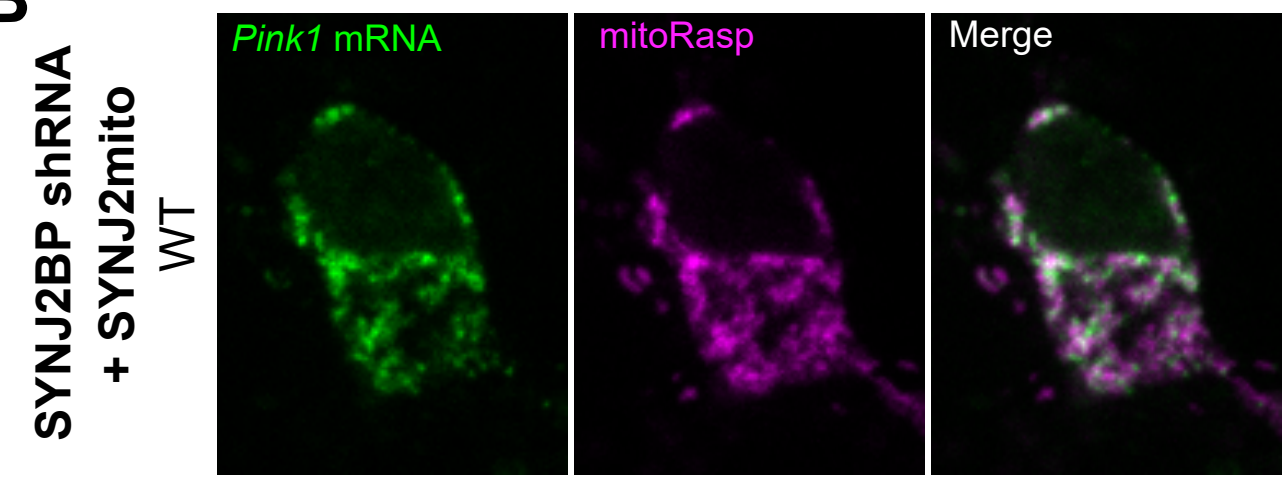

C

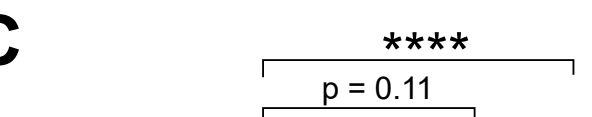

Pink1 mRNA

Control shRNA SYNJ2BP shRNA SYNJ2-mito WT SYNJ2-mito VQL/AAA
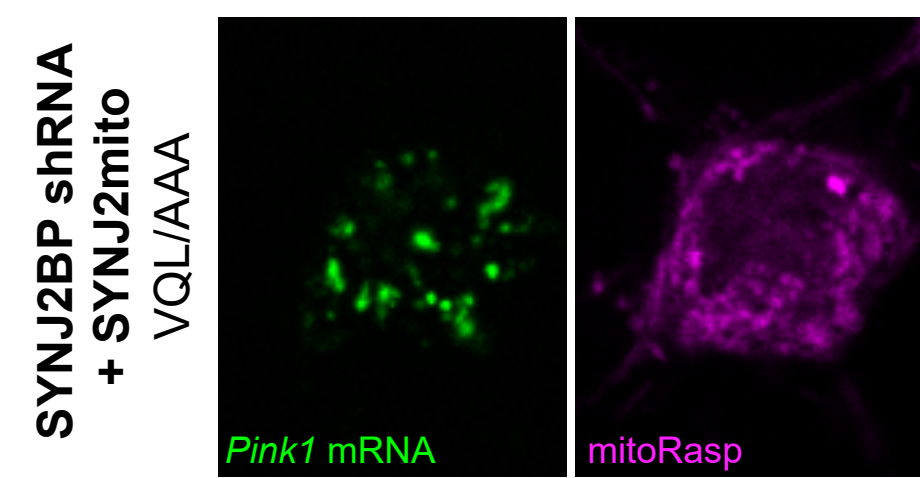

E
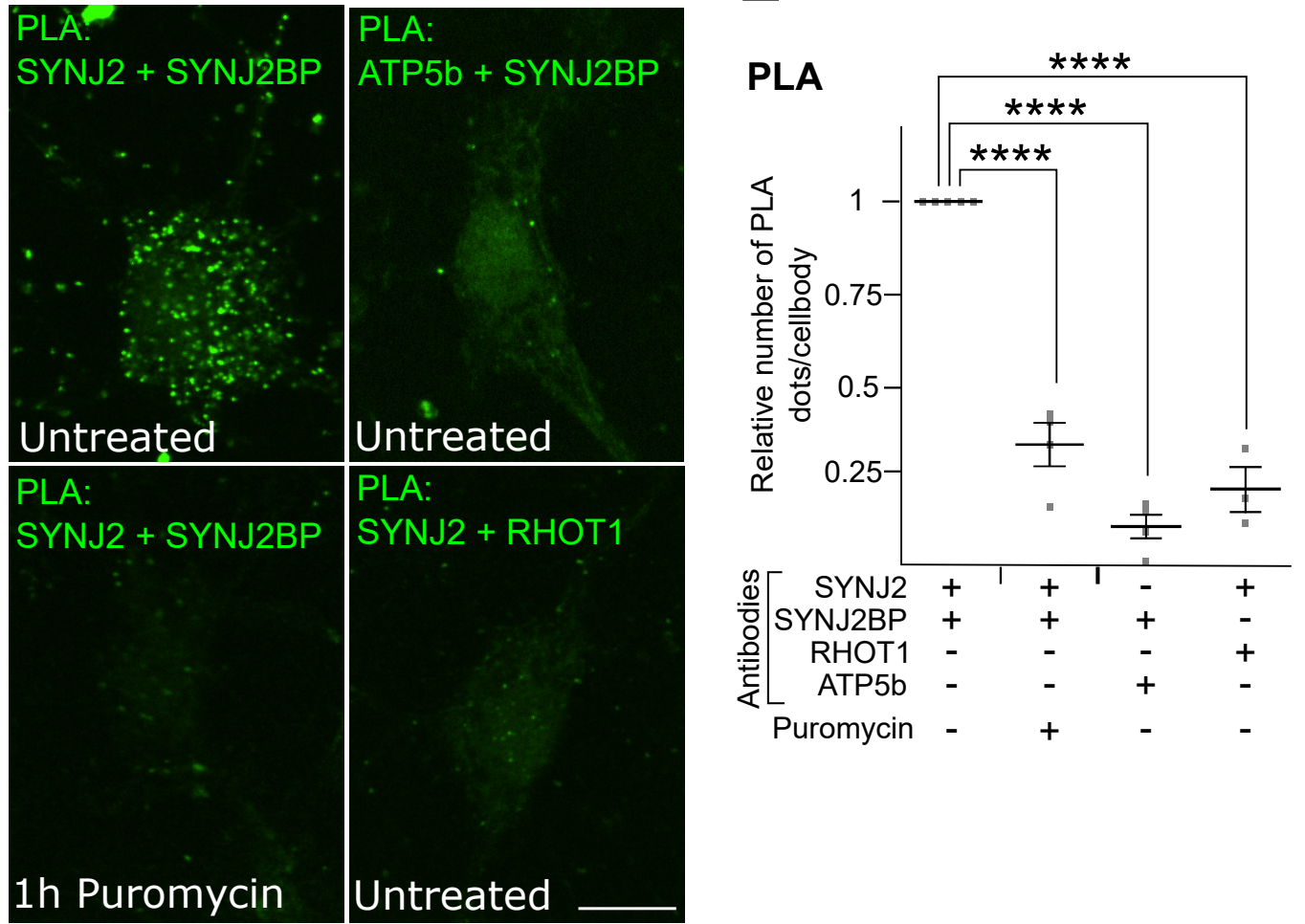


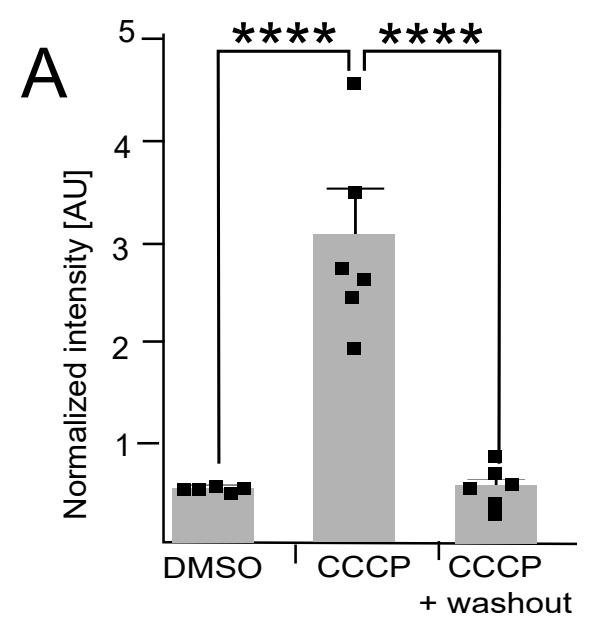

B

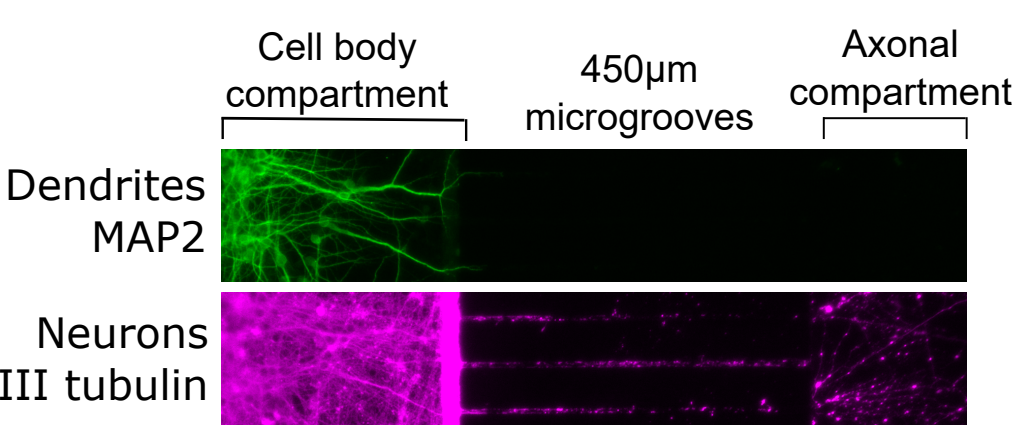

Merge

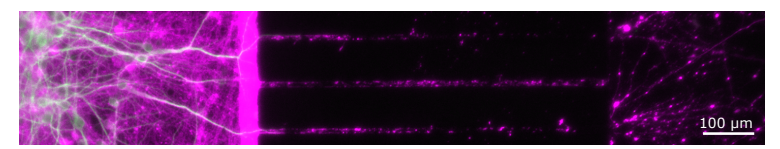

C

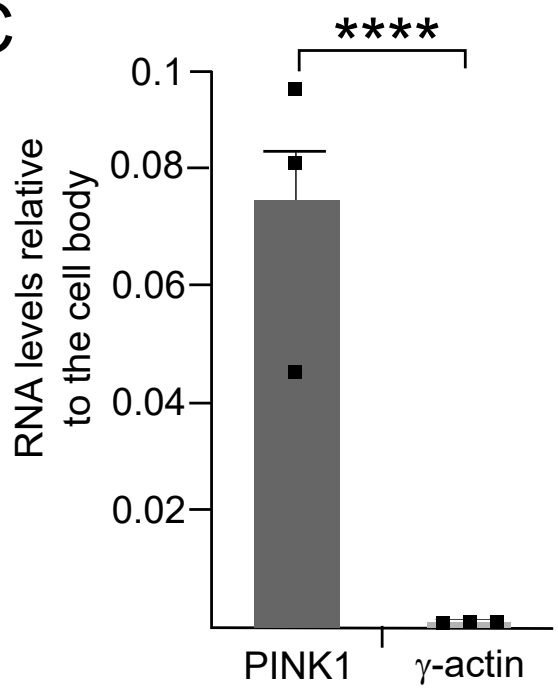


$A$

Pink1 mRNA (splitVenus)

mitoBFP

Identification of axon

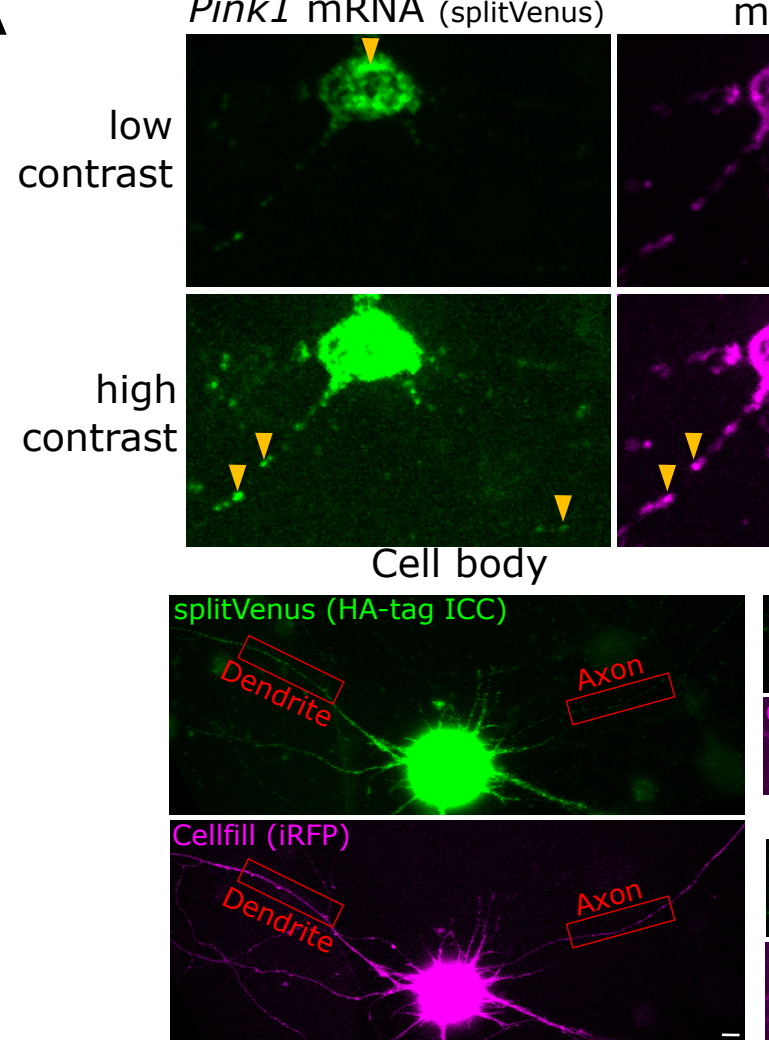

\section{D}
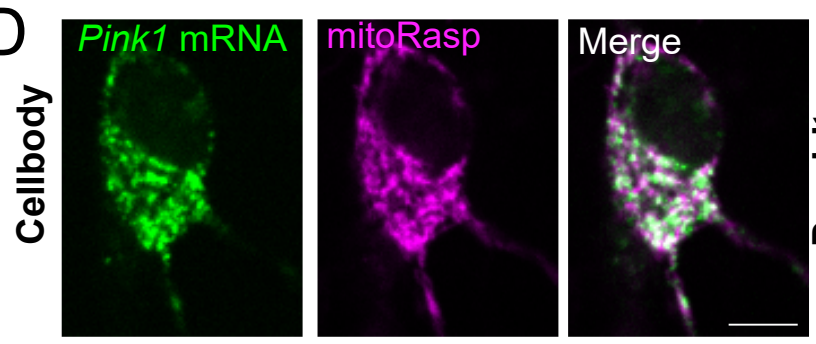

$>750 \mu \mathrm{m}$ from cell body
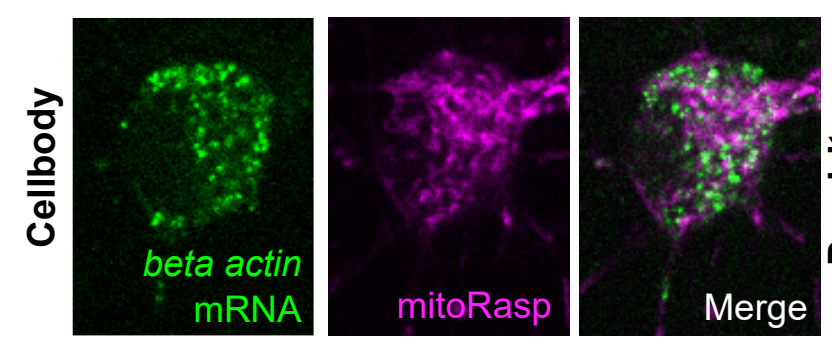

proximal axon

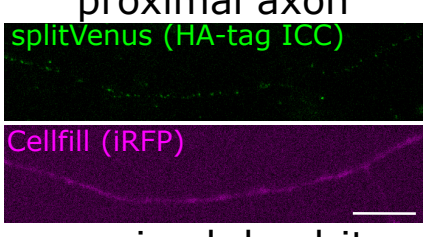

proximal dendrite
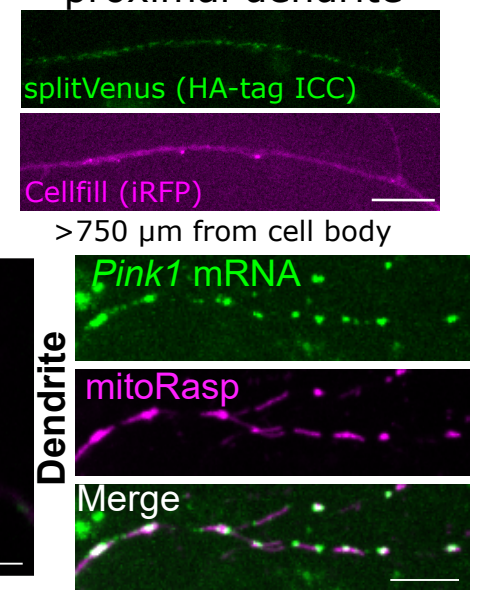

$\mathrm{F}$

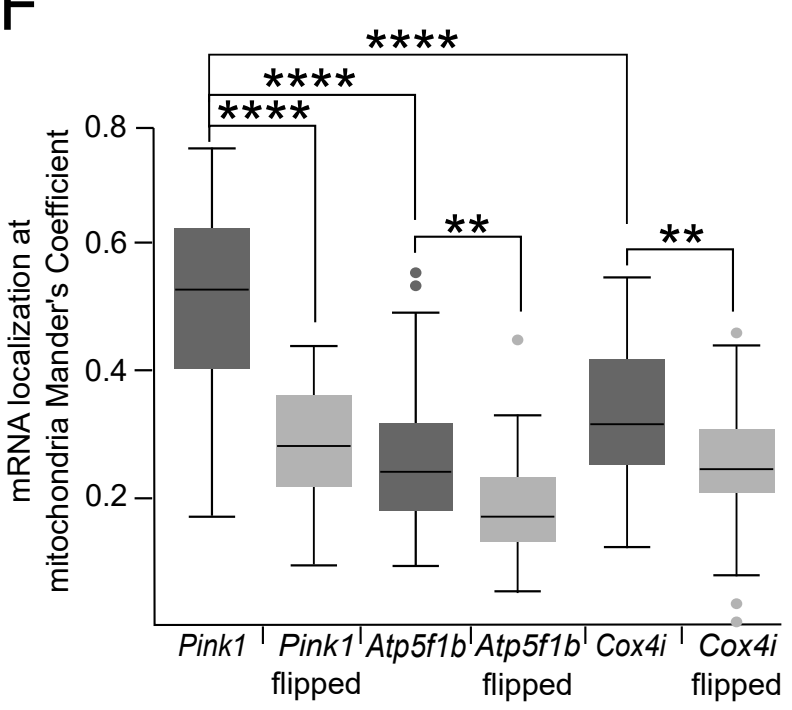

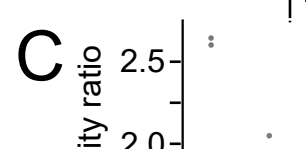

Ankyrin-mCherry
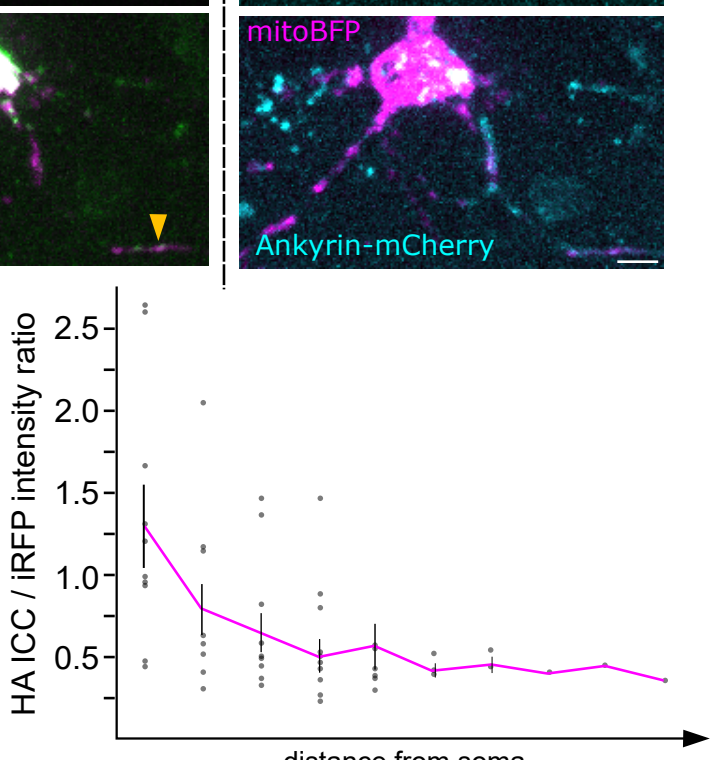

distance from soma
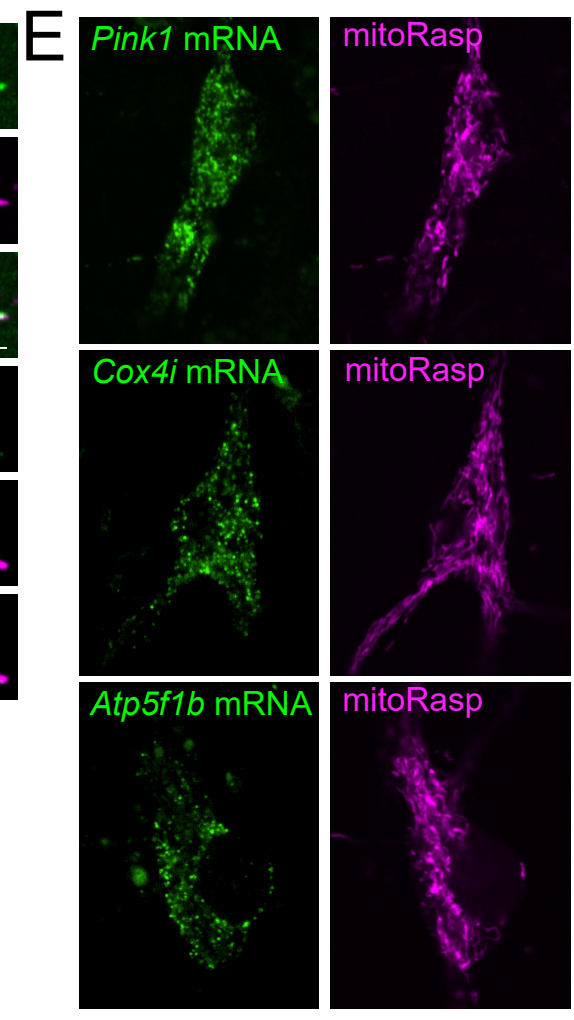

Merge

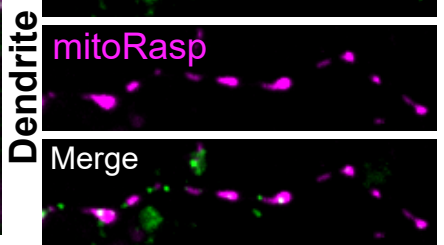

$G$ Pink1 mRNA
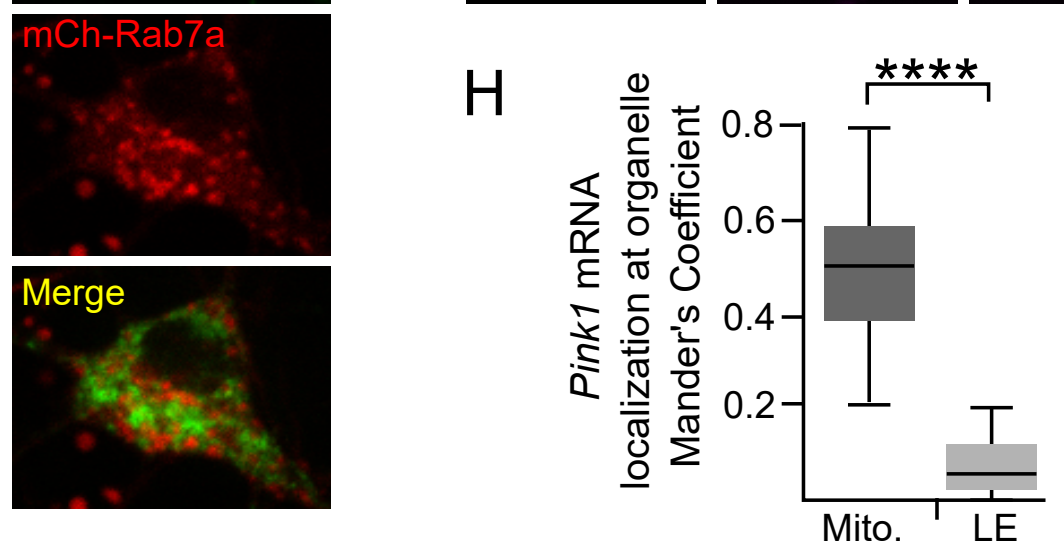
A

Dendrite - live cell imaging

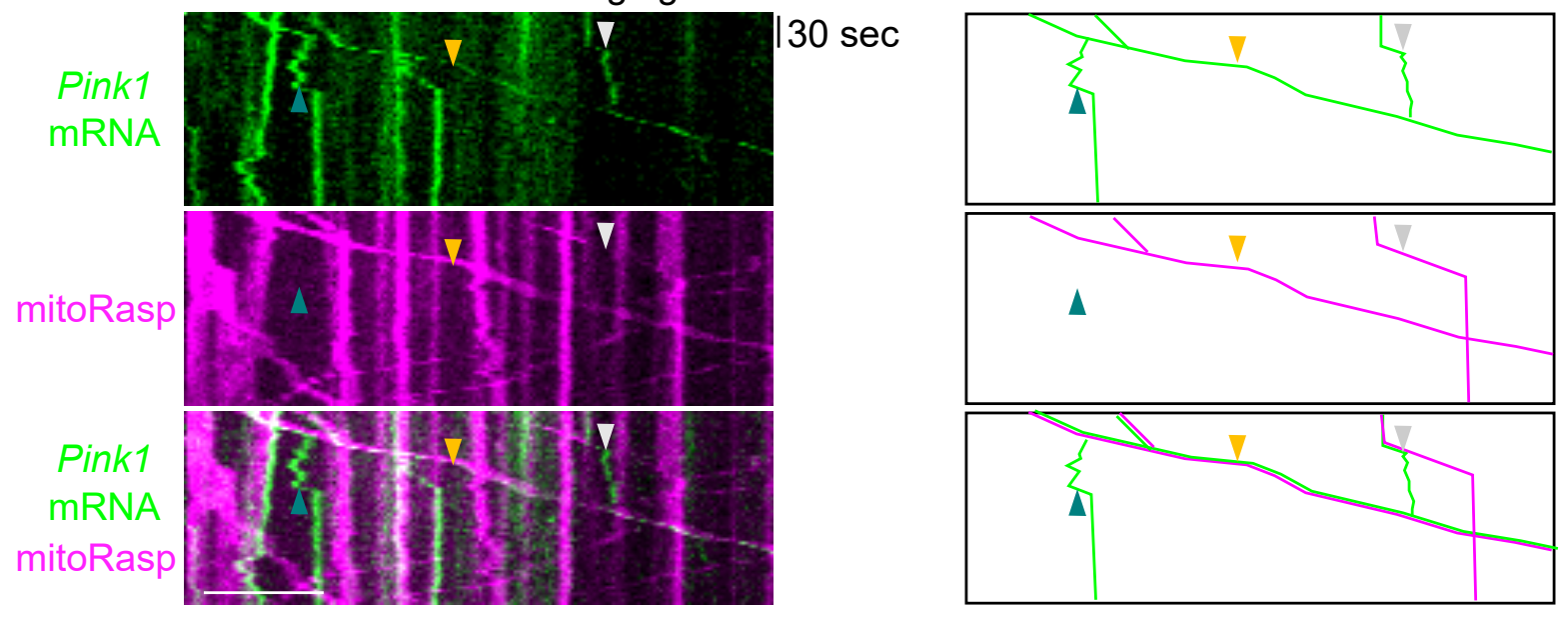

B

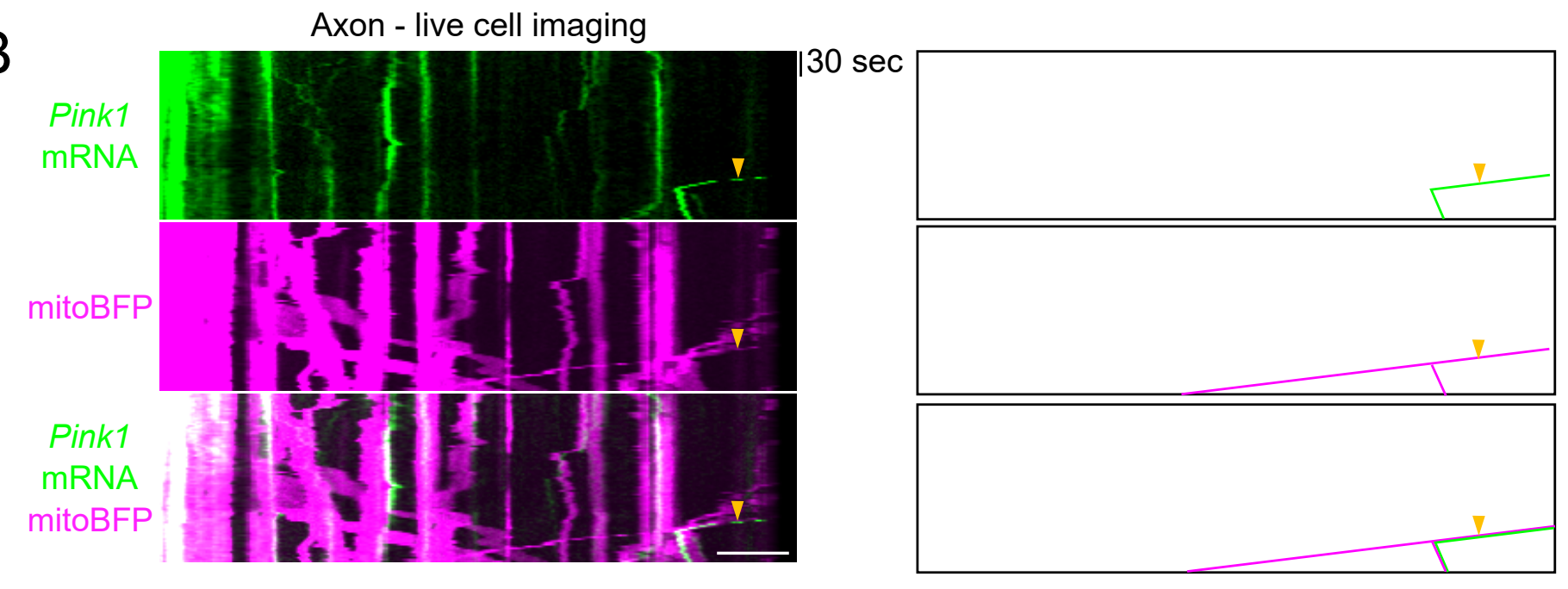


A
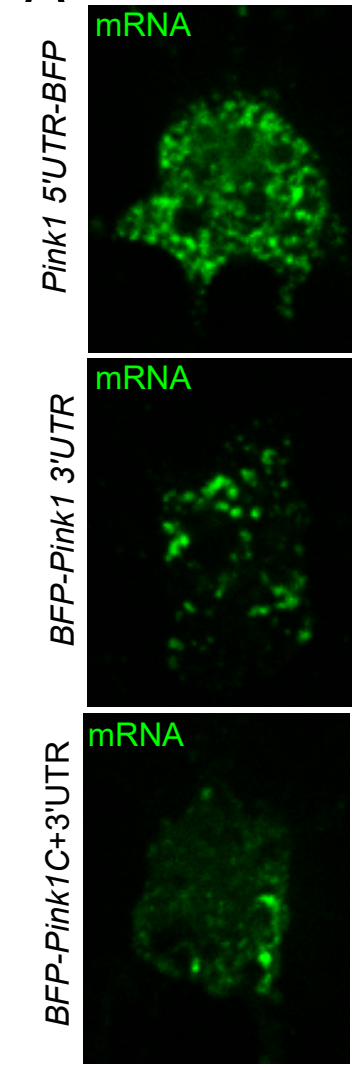
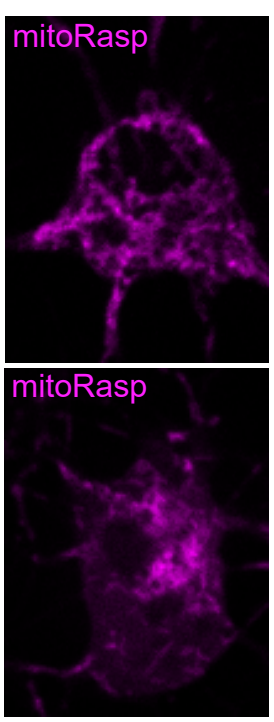

mitoRasp

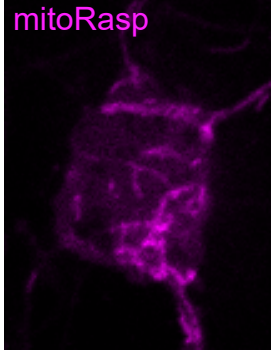

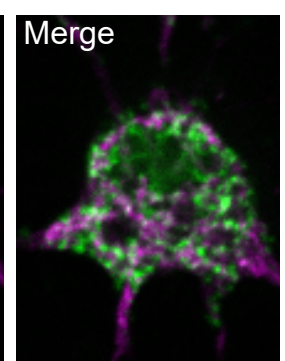

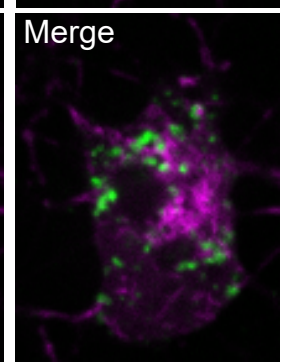

Merge
B

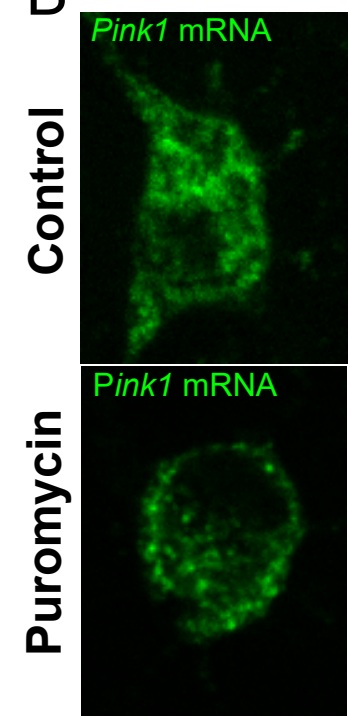

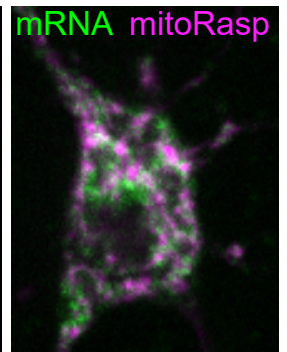

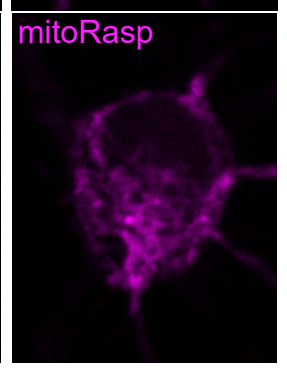

mRNA mitoRasp
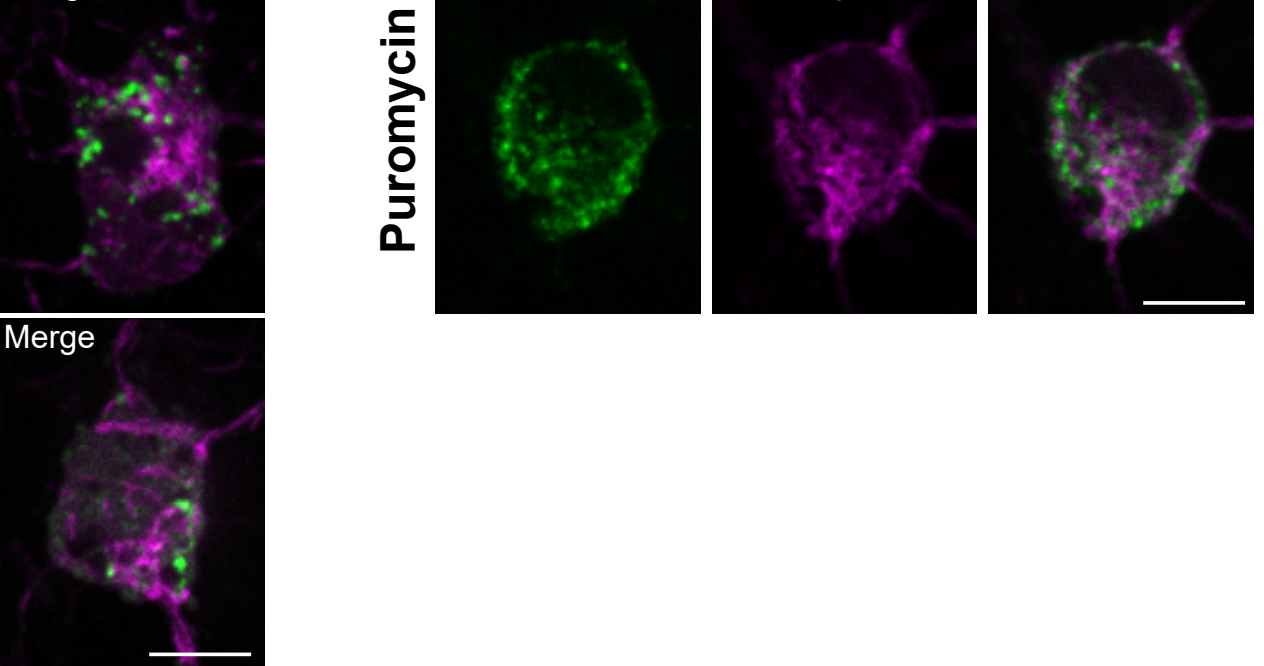
A

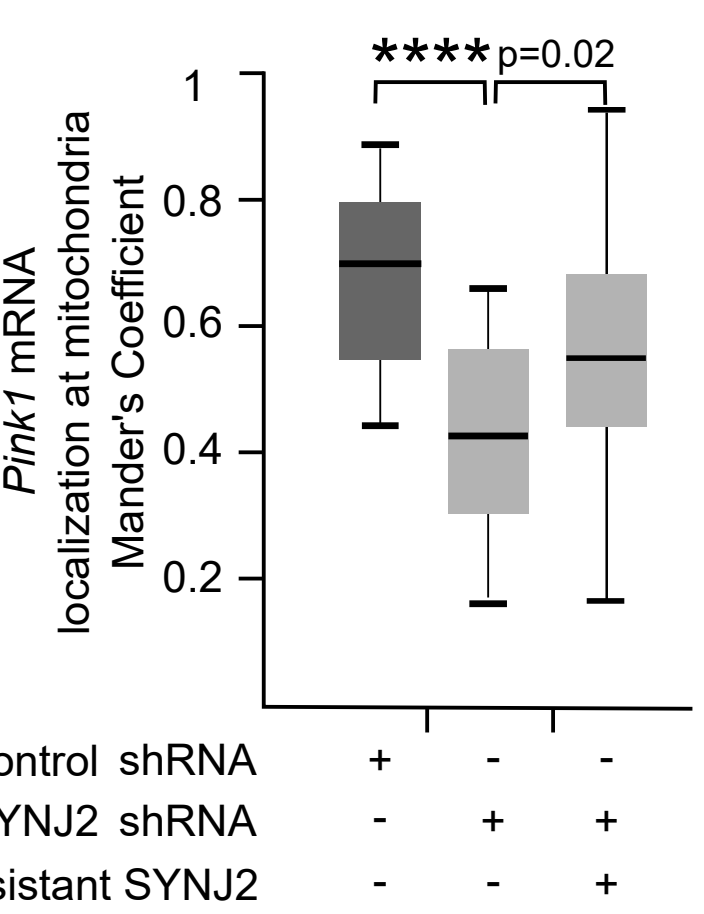

B
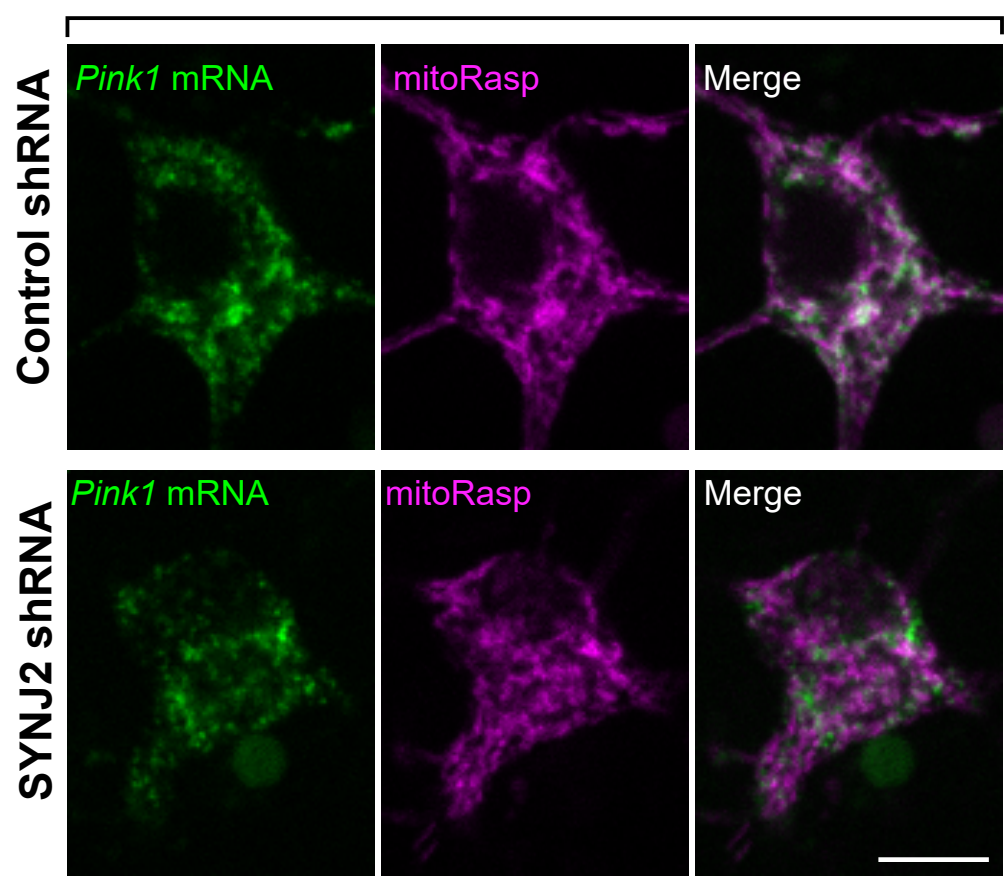

Merge

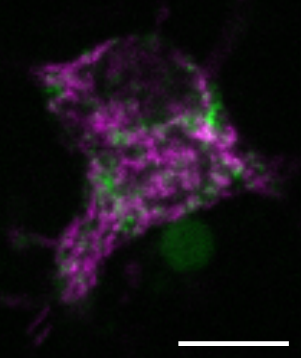


A

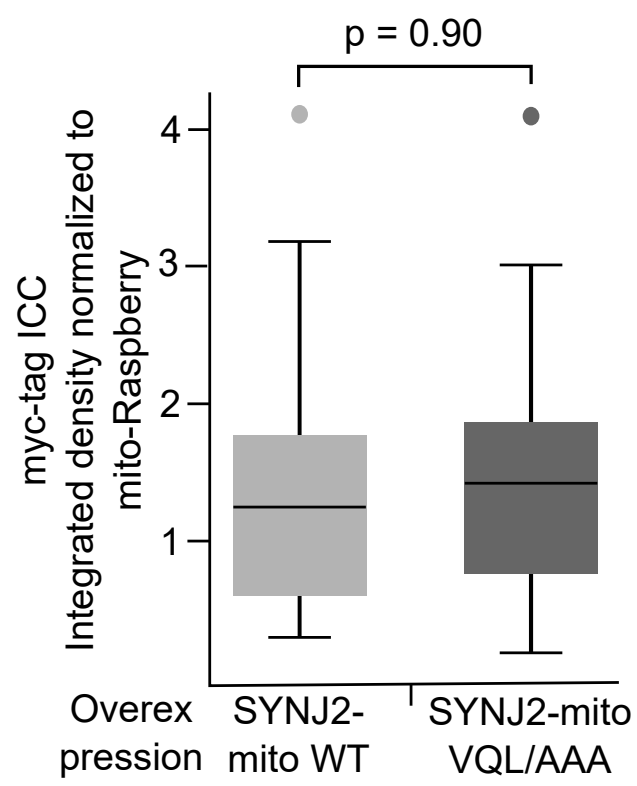

C
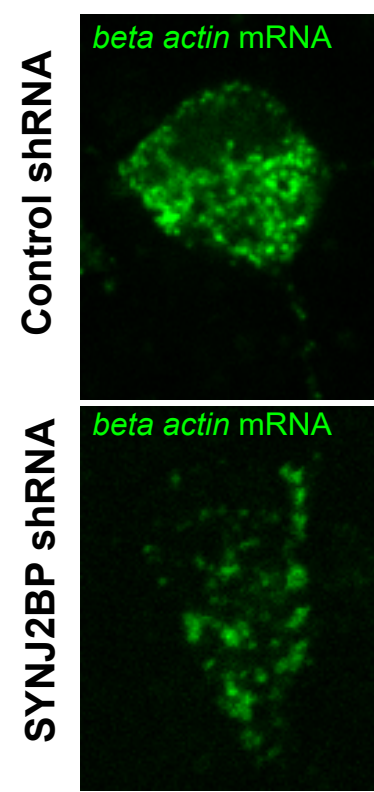

\section{列}
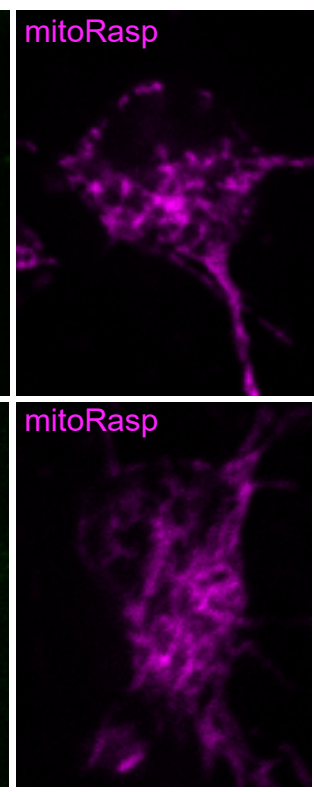

mitoRasp
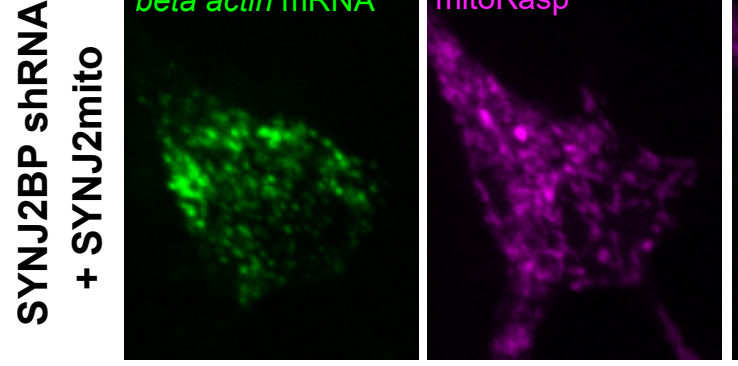

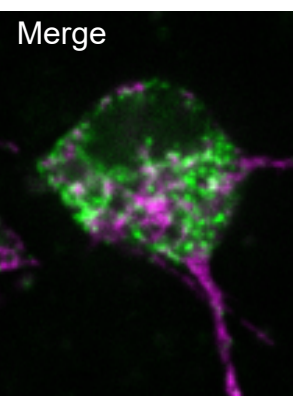

Merge

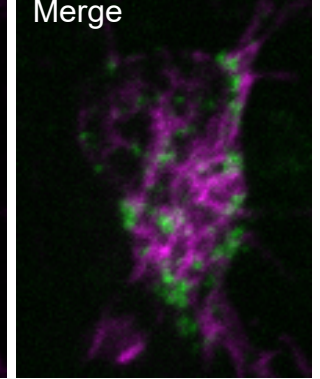

Merge

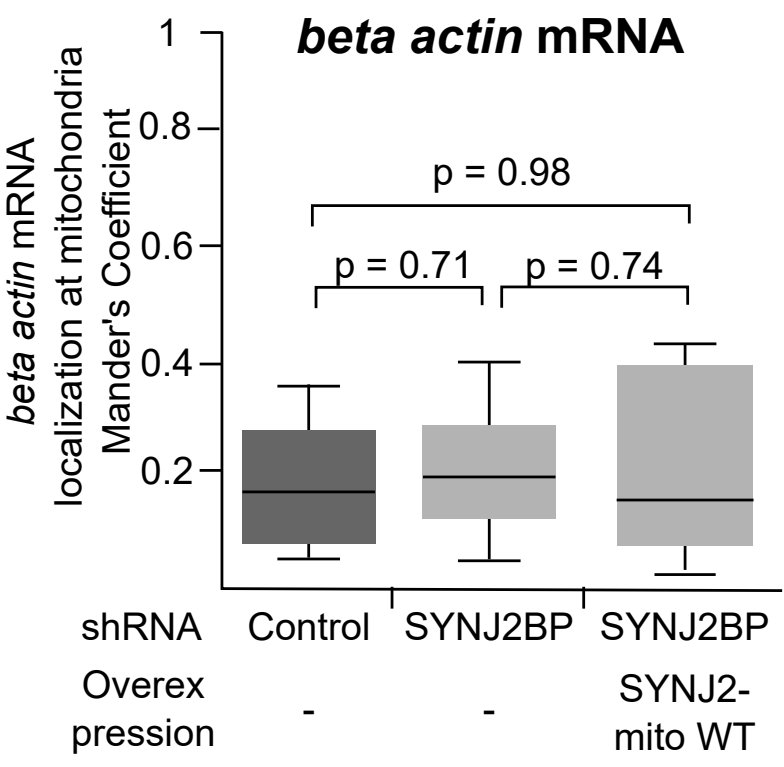

